\title{
Anderson localization of ultrasonic waves in three dimensions
}

\author{
John Page \\ University of Manitoba
}

with Hefei $\mathrm{Hu}^{1}$, Anatoliy Strybulevych ${ }^{1}$, Sergey Skipetrov², Bart van Tiggelen²

${ }^{1}$ University of Manitoba \& 2 Université J. Fourier (Grenoble) 


\begin{tabular}{|c|}
\hline Summer School \\
Mesoscopic Physics in Complex Media \\
Institut d'Etudes Scientifiques de \\
Cargèse \\
12-16 July, 2010 \\
\hline
\end{tabular}

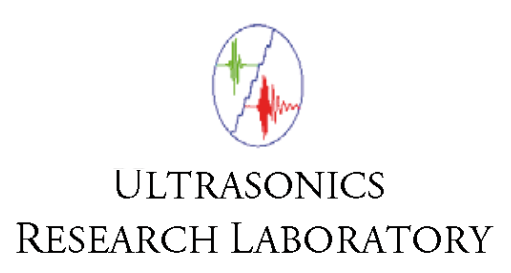

\title{
Anderson localization of ultrasonic waves in three dimensions
}

\author{
John Page \\ University of Manitoba
}

with Hefei Hu${ }^{1}$, Anatoliy Strybulevych ${ }^{1}$, Sergey Skipetrov², Bart van Tiggelen ${ }^{2}$

${ }^{1}$ University of Manitoba \& ${ }^{2}$ Université J. Fourier (Grenoble)

At Manitoba, we use ultrasound to study mesoscopic wave phenomena in complex media, and to probe the physical properties of mesoscopic materials.

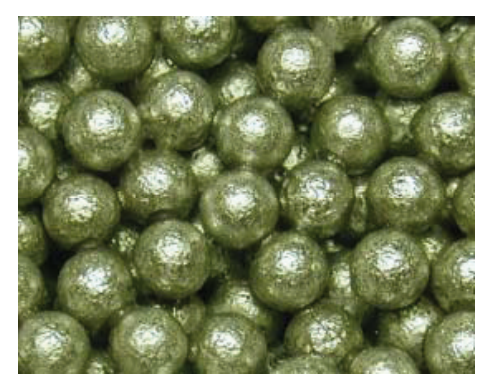

- ballistic and diffusive wave transport in random media

- field fluctuation spectroscopy (DSS, DAWS...)

- wave transport \& focusing in phononic crystals

- ultrasound in complex materials (e.g., soft matter, foods)

www.physics.umanitoba.ca/ jhpage

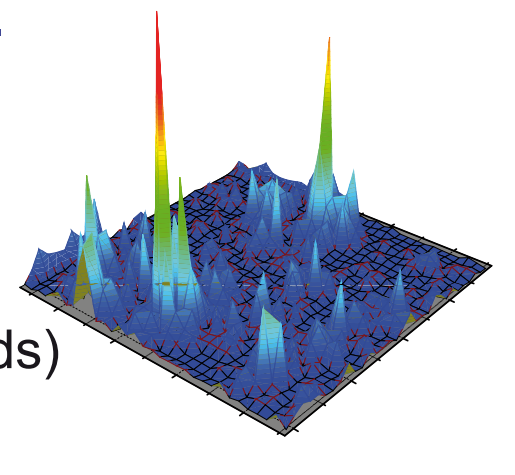




\section{Outline: Anderson Localization of Elastic Waves}

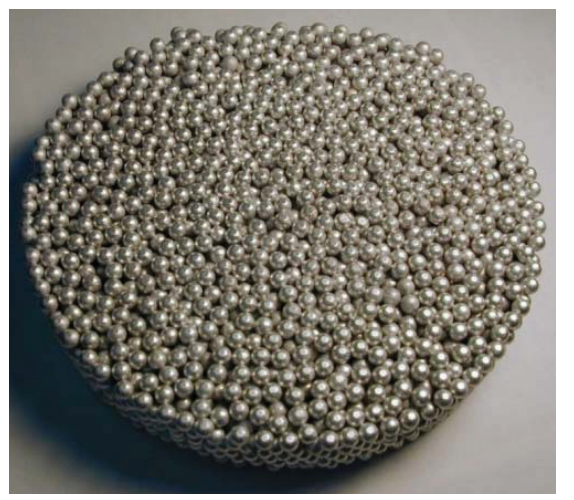

I. Introduction: Brief overview of Anderson localization Why elastic waves?

Our samples \& their basic (wave) properties

II. Time-dependent transmission, $I(t)$

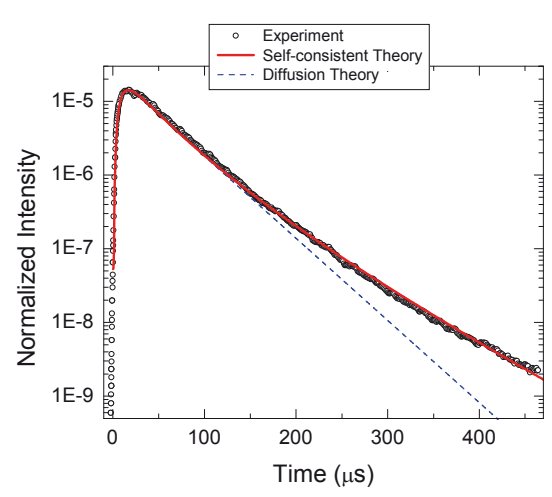

III. Transverse confinement of ultrasonic waves due to localization (“3D transverse localization")

IV. Statistical approach to localization

- non-Rayleigh statistics, variance, multifractality.

V. Conclusions Hu et al., Nature Physics, 4, 945 (Dec, 2008) arXiv:0805.1502 
Introduction: Anderson localization of electrons (quantum particles)

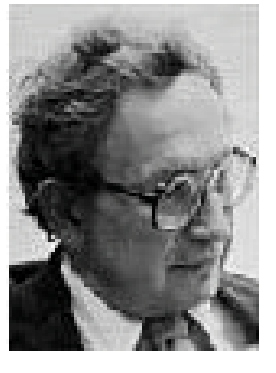

P.W. Anderson 1958

( $\sim 50$ years ago $)$
Schrodinger equation: $V(r)$ varies randomly in space

$$
\left[-\frac{\hbar^{2}}{2 m} \nabla^{2}+V(\mathbf{r})\right] \psi(\mathbf{r})=E \psi(\mathbf{r})
$$
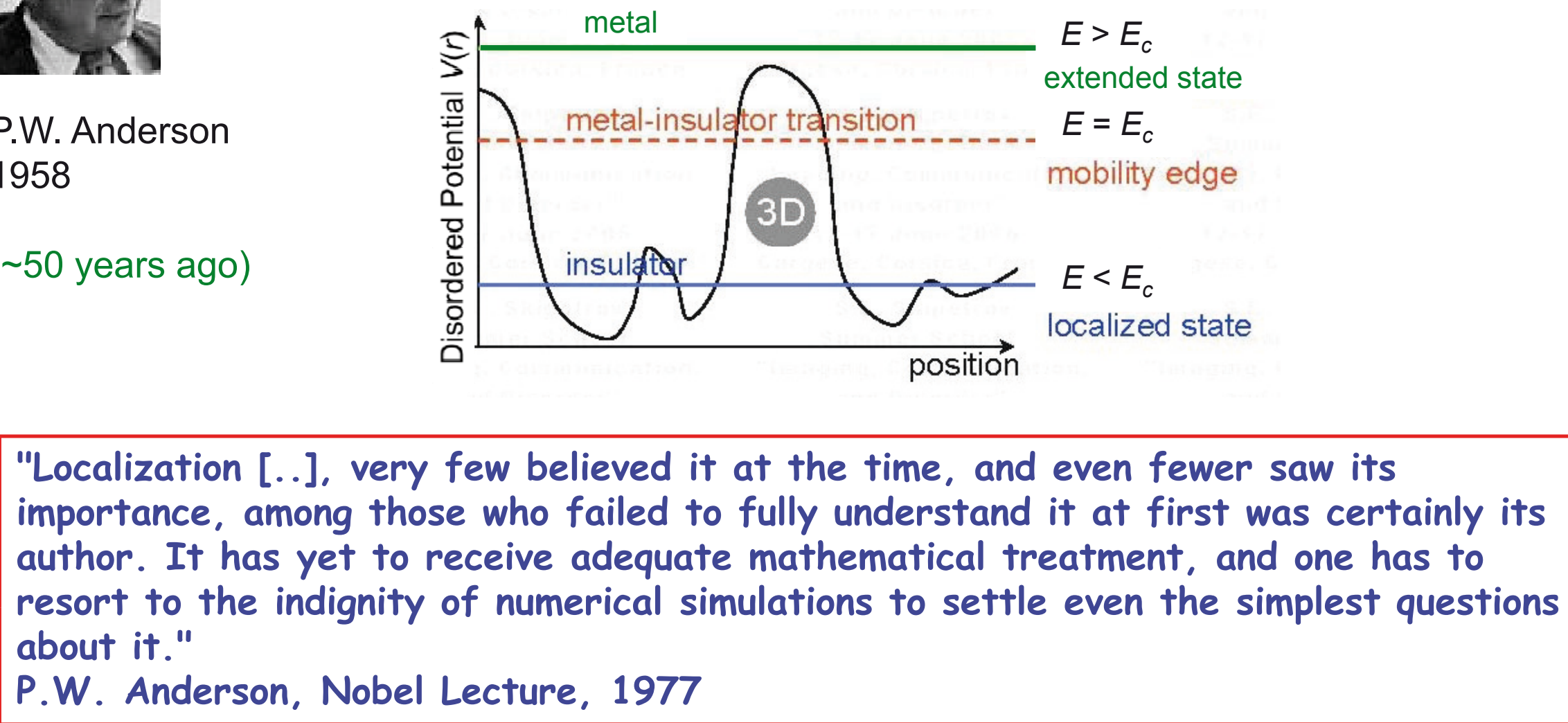

Many theoretical breakthroughs:

e.g. Scaling theory (1979) ( 30 years ago)

Self consistent theory (1980)

\section{Experiments:}

Hampered by interactions and finite temperatures 
Introduction: Anderson localization of electrons (quantum particles)

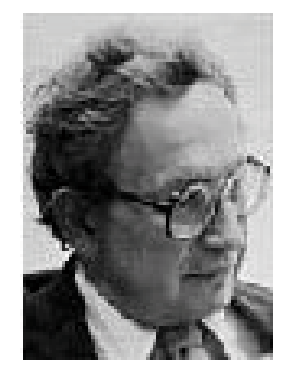

P.W. Anderson 1958

( $\sim 50$ years ago)
Schrodinger equation:

$V(\mathbf{r})$ varies randomly in space

$$
\left[-\frac{\hbar^{2}}{2 m} \nabla^{2}+V(\mathbf{r})\right] \psi(\mathbf{r})=E \psi(\mathbf{r})
$$

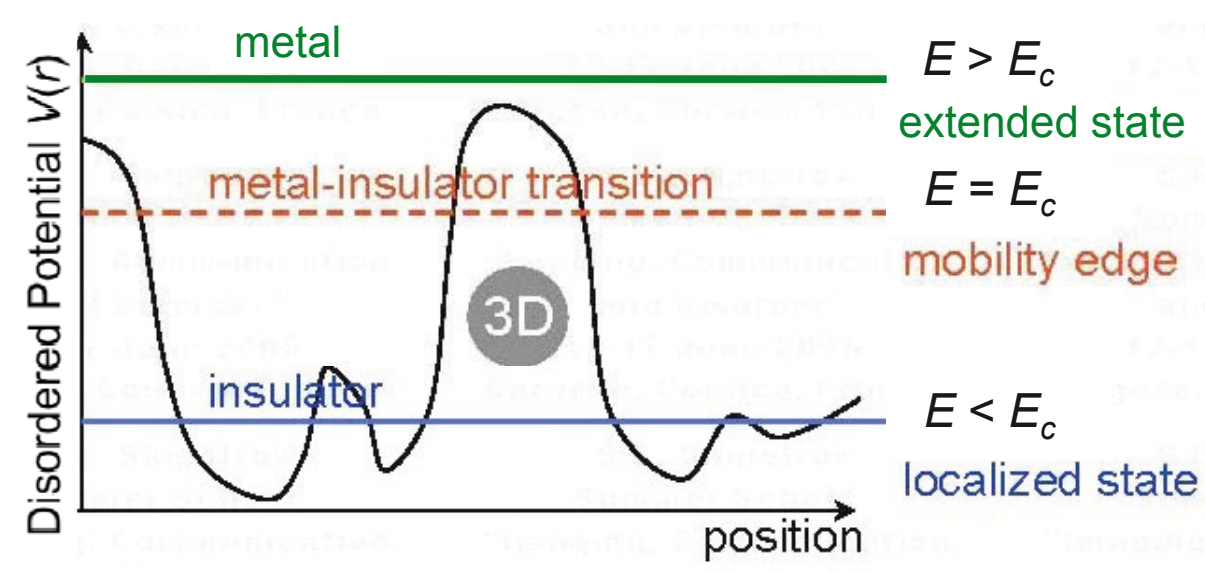

Localization of classical waves (sound or light) e.g., scalar wave equation with disorder:

$\left[-\nabla^{2}+\sigma(r)\right] \psi(r)=\frac{\omega^{2}}{v_{0}^{2}} \psi(r)$

where $\sigma(r)=\frac{\omega^{2}}{v_{0}^{2}}-\frac{\omega^{2}}{v^{2}(r)}$

deviations from a uniform medium with velocity $v_{0}$

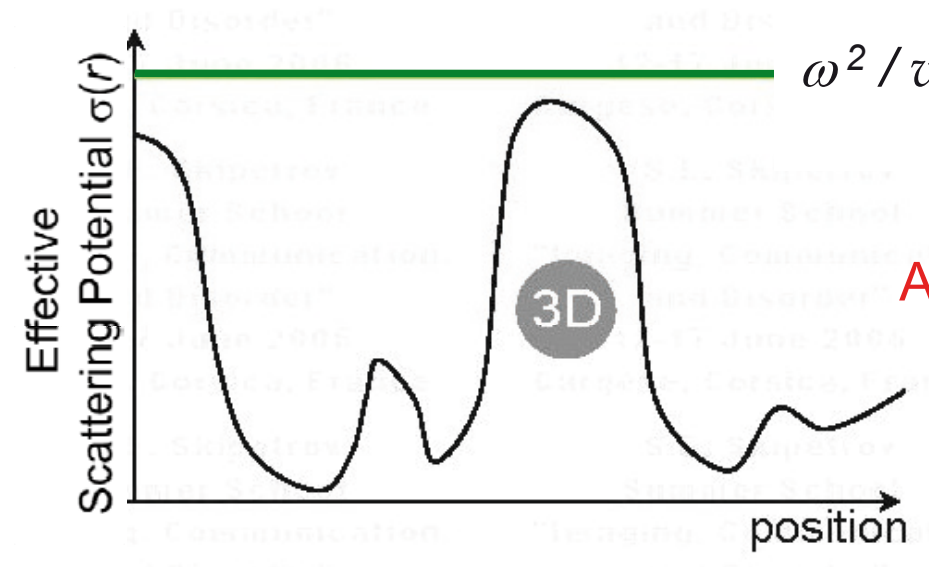

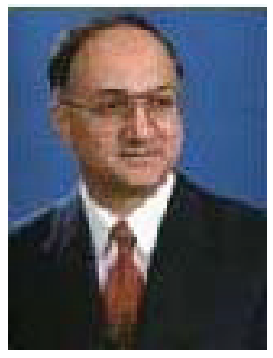

Always!

Sajeev John 1983

( $\sim 25$ years ago) 


\section{Previous acoustic experiments in 1D:}

A disordered chain of masses and springs - measure the transverse displacements for different amounts of disorder [He and Maynard, PRL, 57, 3171]:

Diagonal disorder: vary the positions of the masses.

Off-diagonal disorder: vary the sizes of the masses.

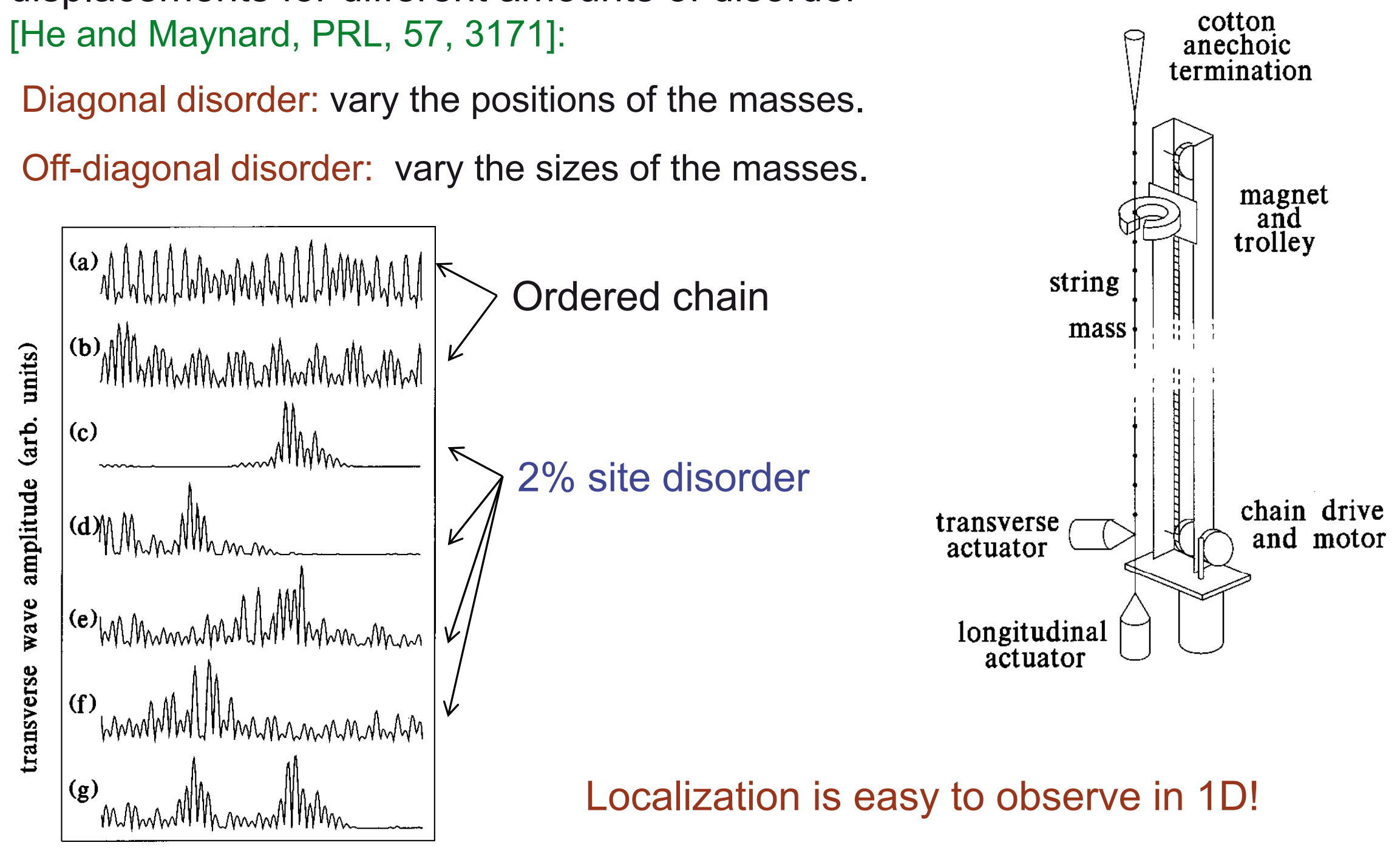

position along wire 


\section{Previous ultrasonic experiments in 2D:}

Ultrasound in a disordered plate with random slots [Weaver, Wave Motion, 12, 129-142(1990) ; Lobkis and Weaver, J. Acoust. Soc. Am. 124, 3528 (2008)]:

Measure (with 4 small transducers):

$$
R(r, t, f)=\frac{\left[E_{1 b} E_{2 a} E_{2 b} E_{1 a}\right]^{1 / 4}}{\left[E_{12} E_{a b}\right]^{1 / 2}}=\frac{E_{\text {distant }}}{E_{\text {adjacent }}}
$$

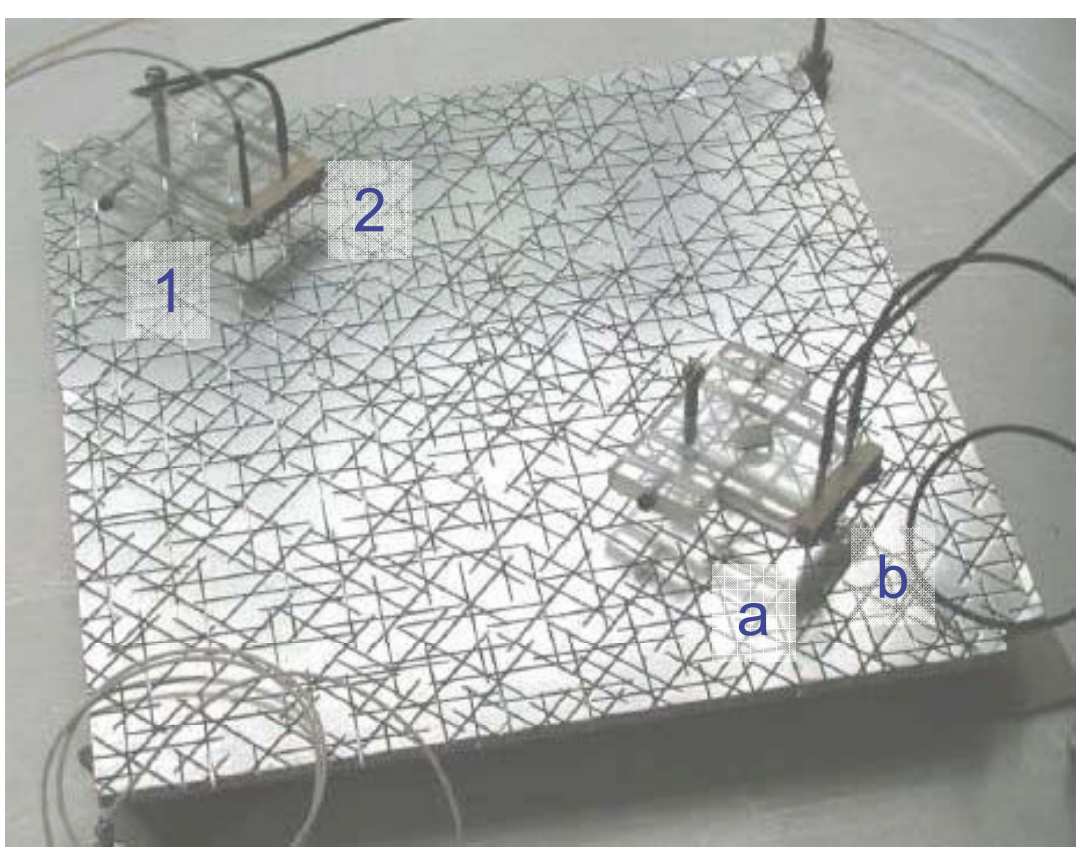

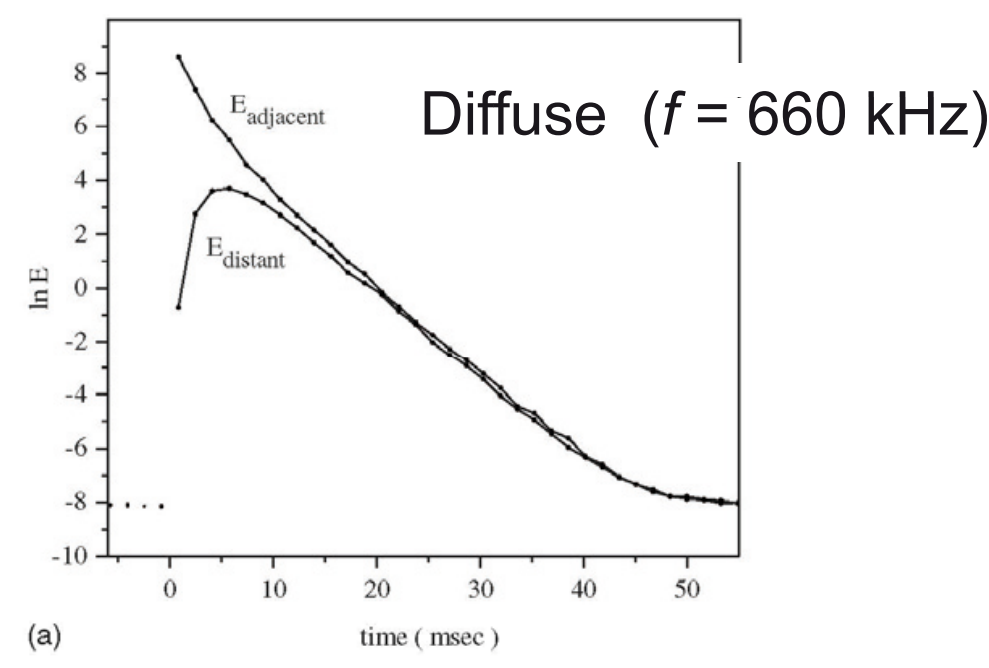

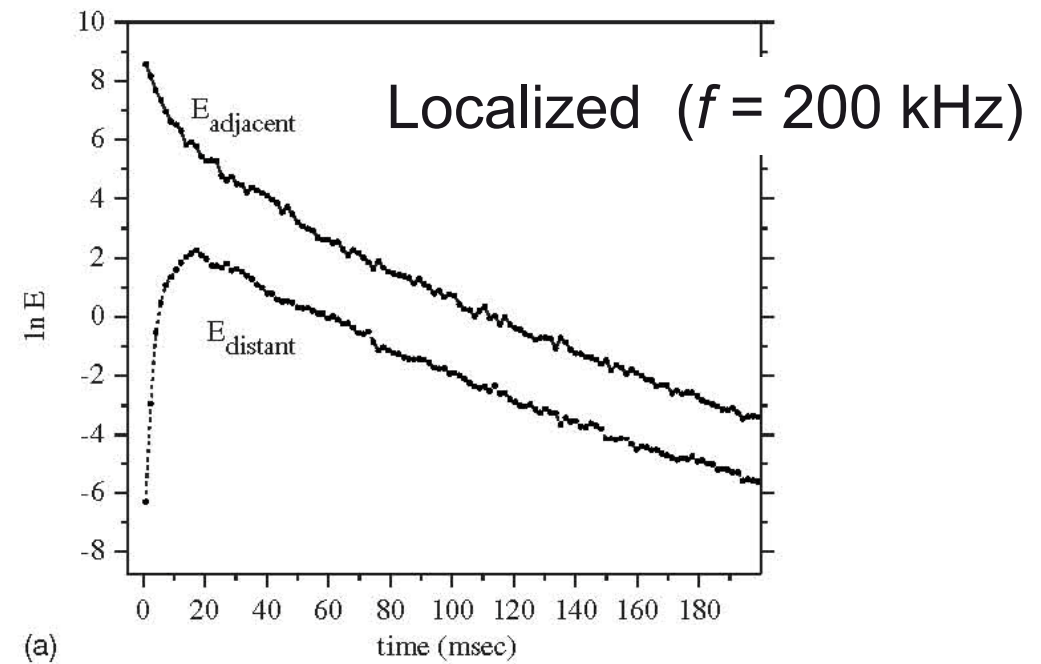




\section{Previous ultrasonic experiments in 2D:}

Ultrasound in a disordered plate with random slots [Weaver, Wave Motion, 12, 129-142(1990) ; Lobkis and Weaver, J. Acoust. Soc. Am. 124, 3528 (2008)]:

Measure (with 4 small transducers):

$$
R(r, t, f)=\frac{\left[E_{1 b} E_{2 a} E_{2 b} E_{1 a}\right]^{1 / 4}}{\left[E_{12} E_{a b}\right]^{1 / 2}}=\frac{E_{\text {distant }}}{E_{\text {adjacent }}}
$$
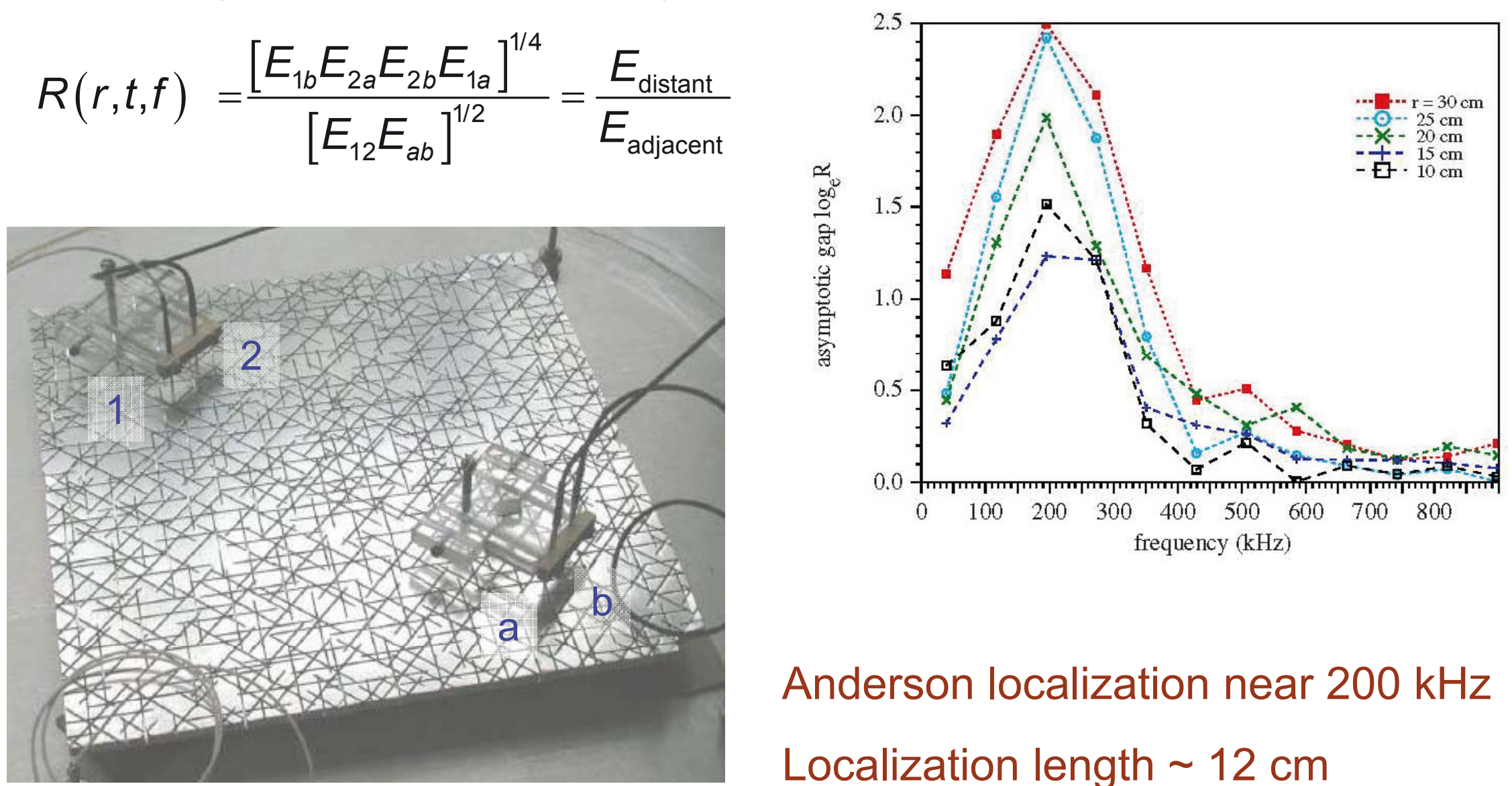

Anderson localization near $200 \mathrm{kHz}$

Localization length $\sim 12 \mathrm{~cm}$ 


\section{Previous experiments with light in 3D:}

Exponential scaling of the average transmission (for monochromatic waves) with thickness L. [Wiersma et al., Nature 390, 671 (1997)]

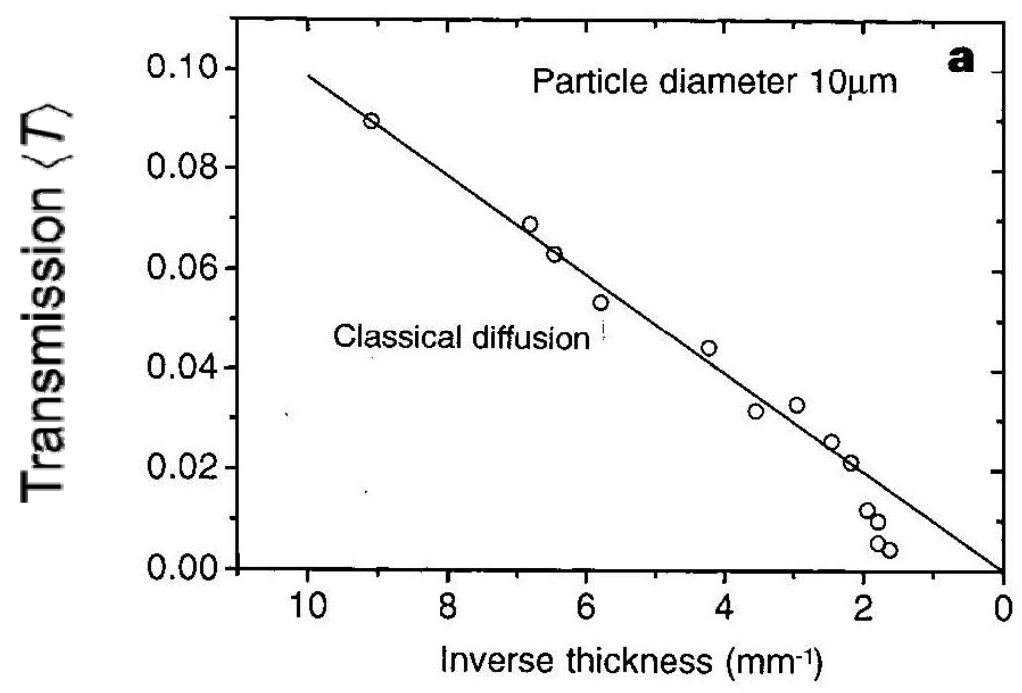

Diffuse regime:

$$
\langle T\rangle \propto \frac{\ell^{*}}{L}
$$

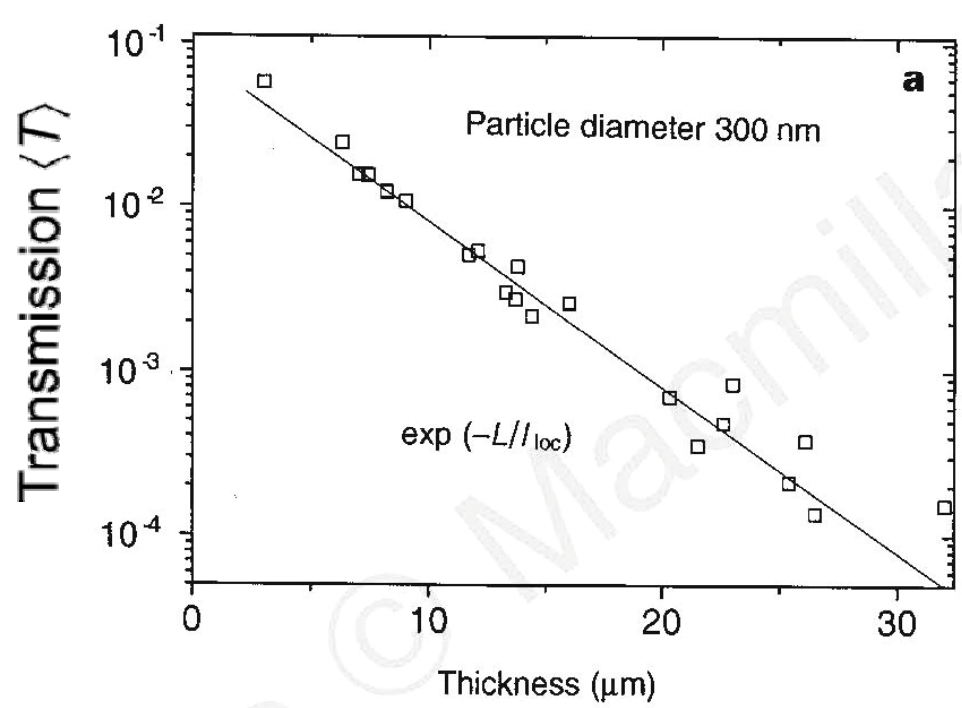

Localized regime

$$
\langle T\rangle \propto \exp \left[-\frac{L}{\xi}\right]
$$

- Difficult to distinguish from effects of absorption ( $\left.\propto \exp \left[-L / \ell_{a}\right]\right)$ 


\section{Previous experiments with microwaves in quasi-1D:}

Enhanced fluctuations of total transmission.

[Chabanov et al., Nature 404, 850 (2000)]

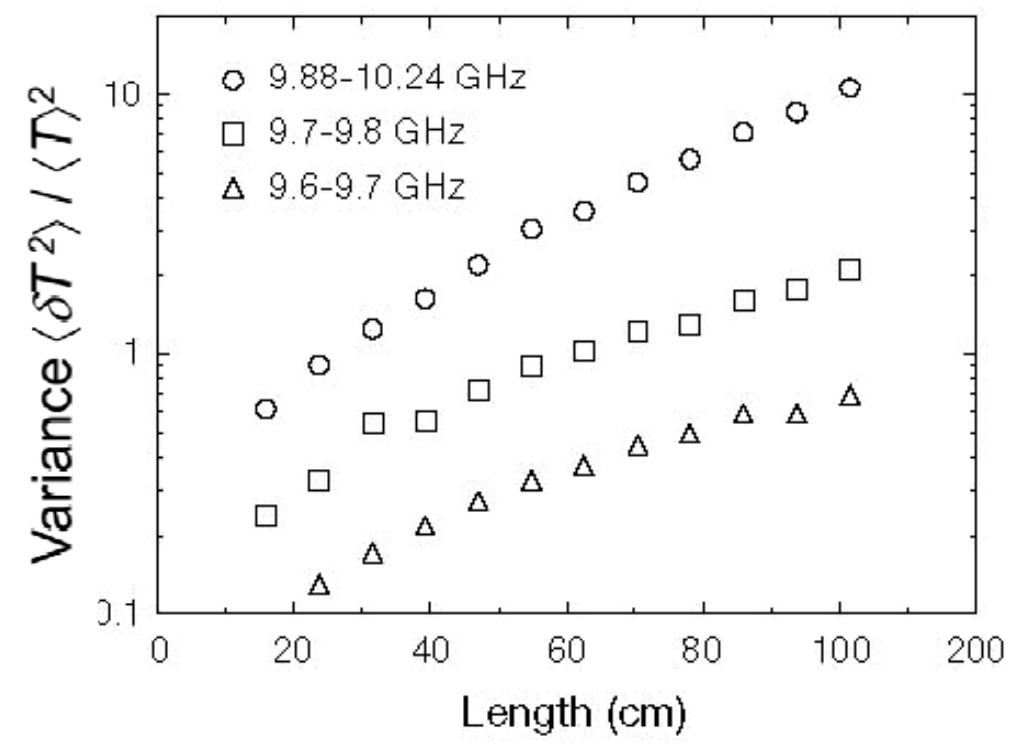

Diffuse regime:

$$
\frac{\left\langle\delta T^{2}\right\rangle}{\langle T\rangle^{2}} \ll 1
$$

Localized regime

$$
\frac{\left\langle\delta T^{2}\right\rangle}{\langle T\rangle^{2}}>\text { const } \sim 1
$$

- Chabanov et al. proposed that this criterion for localization is independent of absorption, but their experiments were limited to quasi-1-dimensional samples. 
More recent experiments with light in 3D:

Time-dependent transmission through thick samples of $\mathrm{TiO}_{2}$ particles [Störzer et al., PRL 96, 063904 (2006)]

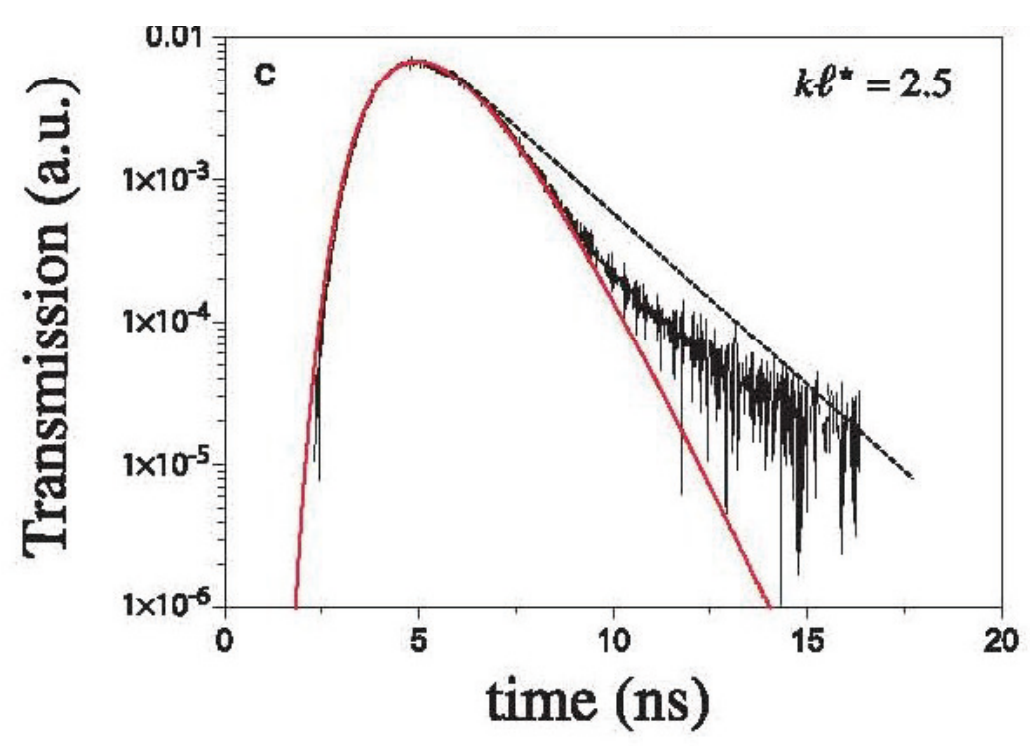

Non-exponential tail at long times:

interpreted as a slowing down of diffusion with propagation time due to localization.

Current status ( $\sim 50$ years after Anderson's discovery):

- The subject is more alive than ever!

- Activity in optics, microwaves, acoustics, seismic waves, and atomic matter waves. 
Question: Can we convincing observe the localization of ultrasound due to disorder in $3 \mathrm{D}$, and, if so, can we learn something new?

NB Scaling theory $\Rightarrow$ Only in $3 D$ is there a real transition from extended to localized modes (i.e., a mobility edge).

Weak disorder $(k \ell>>1)$ :

\section{Diffuse propagation}

$D_{B}=1 / 3 v_{E} \ell_{B}^{*}$ (neglect interference)

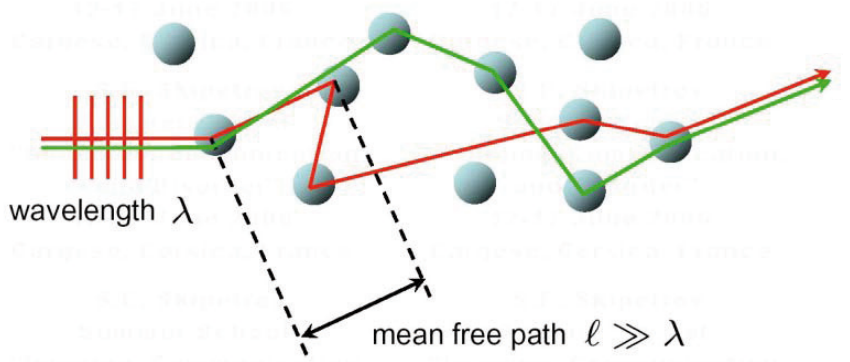

Strong disorder $(k \ell \sim 1)$ :

Anderson localization (interference is important!)

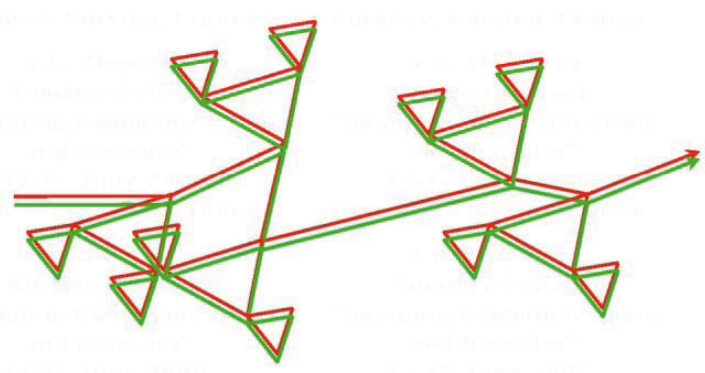

e.g., After a short pulse of ultrasound is incident on the medium...

Energy density spreads diffusively from the source
Localization length $\xi$

Energy remains localized the vicinity of the source 
Searching for suitable 3D materials...

\section{A) An acoustic 3D system -}

suspensions of glass spheres in a liquid:

- A model system for studying the ballistic and diffusive transport of ultrasound.
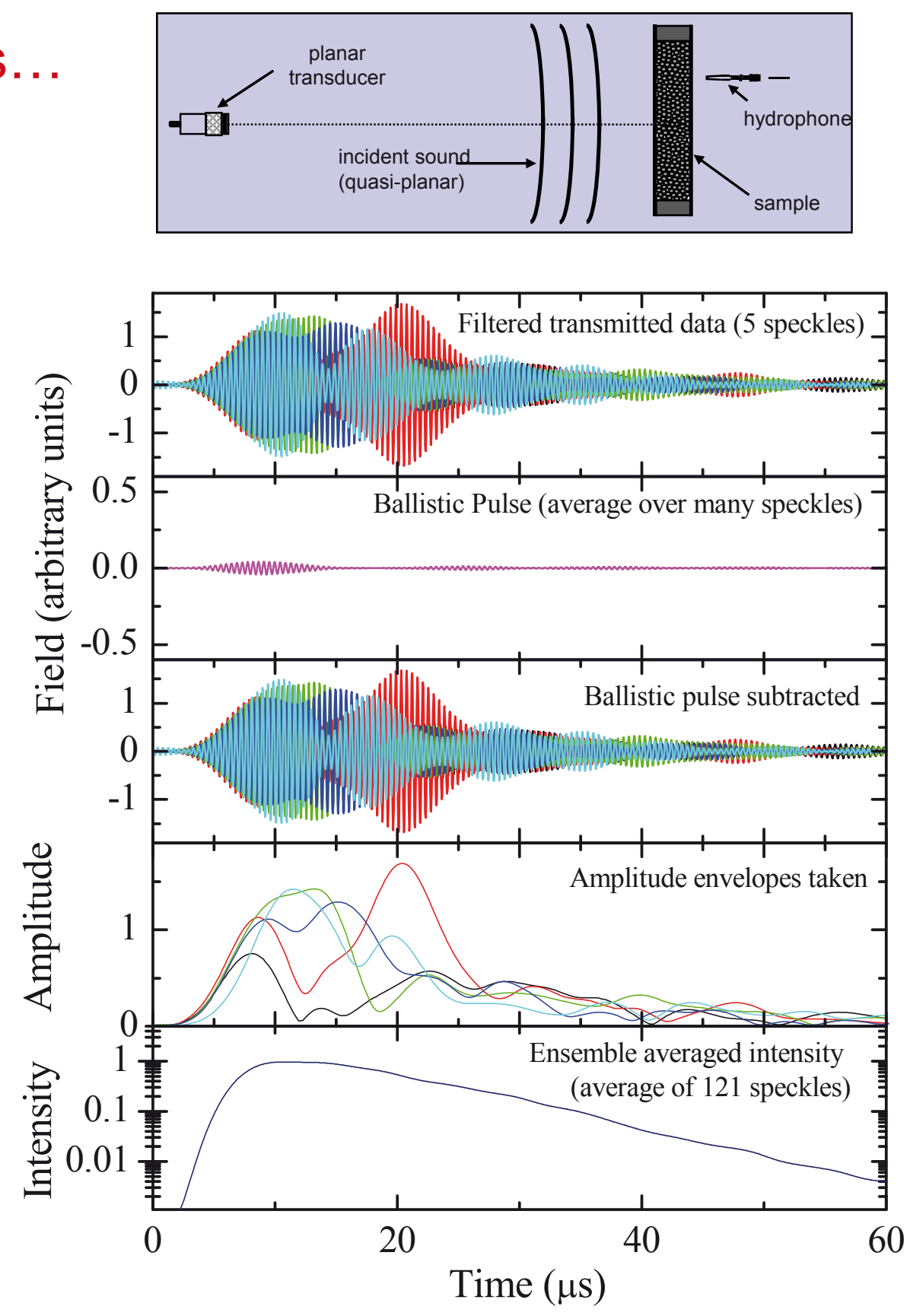
Searching for suitable 3D materials...

An acoustic 3D system - suspensions of glass spheres in a liquid:

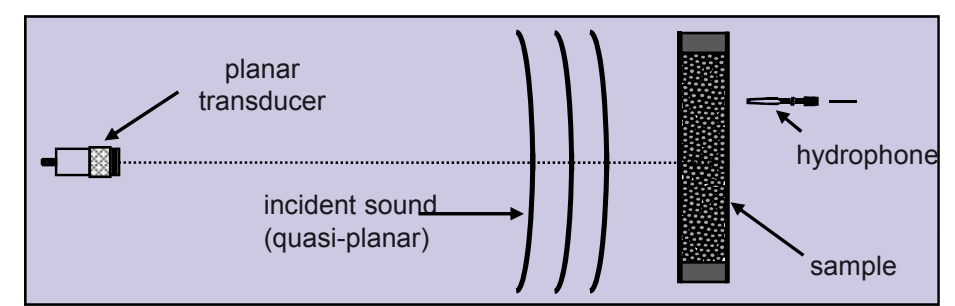

- A model system for studying the ballistic and diffusive transport of ultrasound.

Ballistic transport: from the weak coherent pulse measure :

- phase velocity: $v_{p}=\omega / k$

- group velocity: $v_{g}=\mathrm{d} \omega / \mathrm{d} k$

- scattering mean free path, $\ell$ :

$$
I=I_{0} \exp [-L / \ell]
$$

(Page et al., Science 271, 614 (1996))

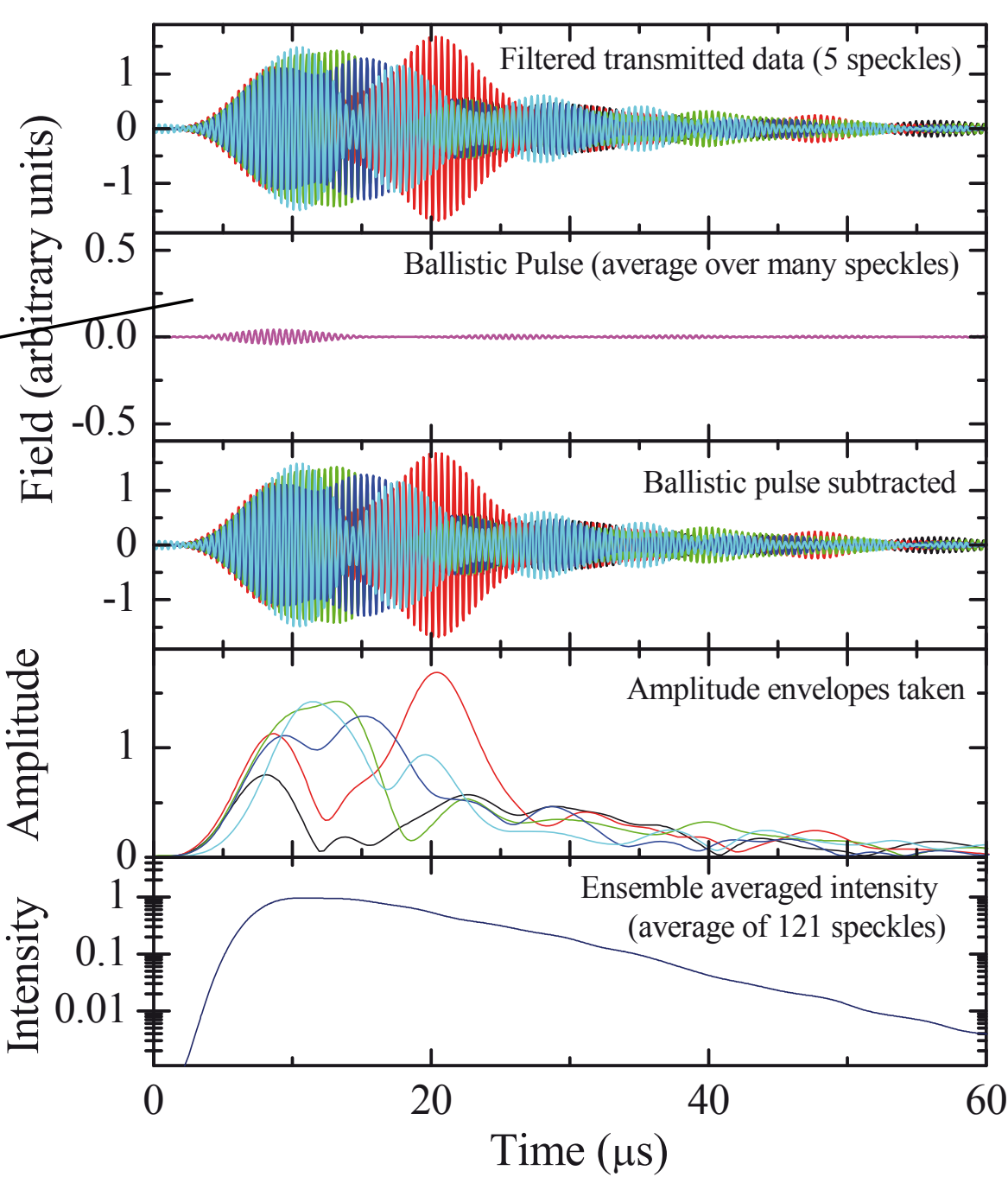


Searching for suitable 3D materials...

An acoustic 3D system - suspensions of glass spheres in a liquid:

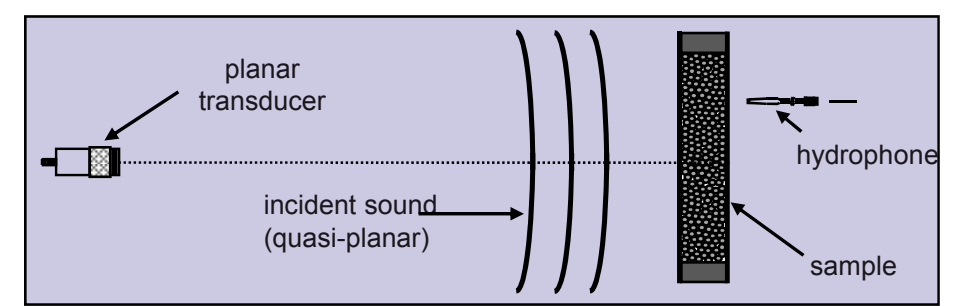

- A model system for studying the ballistic and diffusive transport of ultrasound.

Diffusive transport:

By fitting $I(t)$ to the predictions of the diffusion approximation, measure:

- diffusion coefficient: $D_{B}=\frac{1}{3} v_{E} \ell^{*}$

- absorption time: $\tau_{a}$

(Page et al., PRE 52, 3106 (1995))

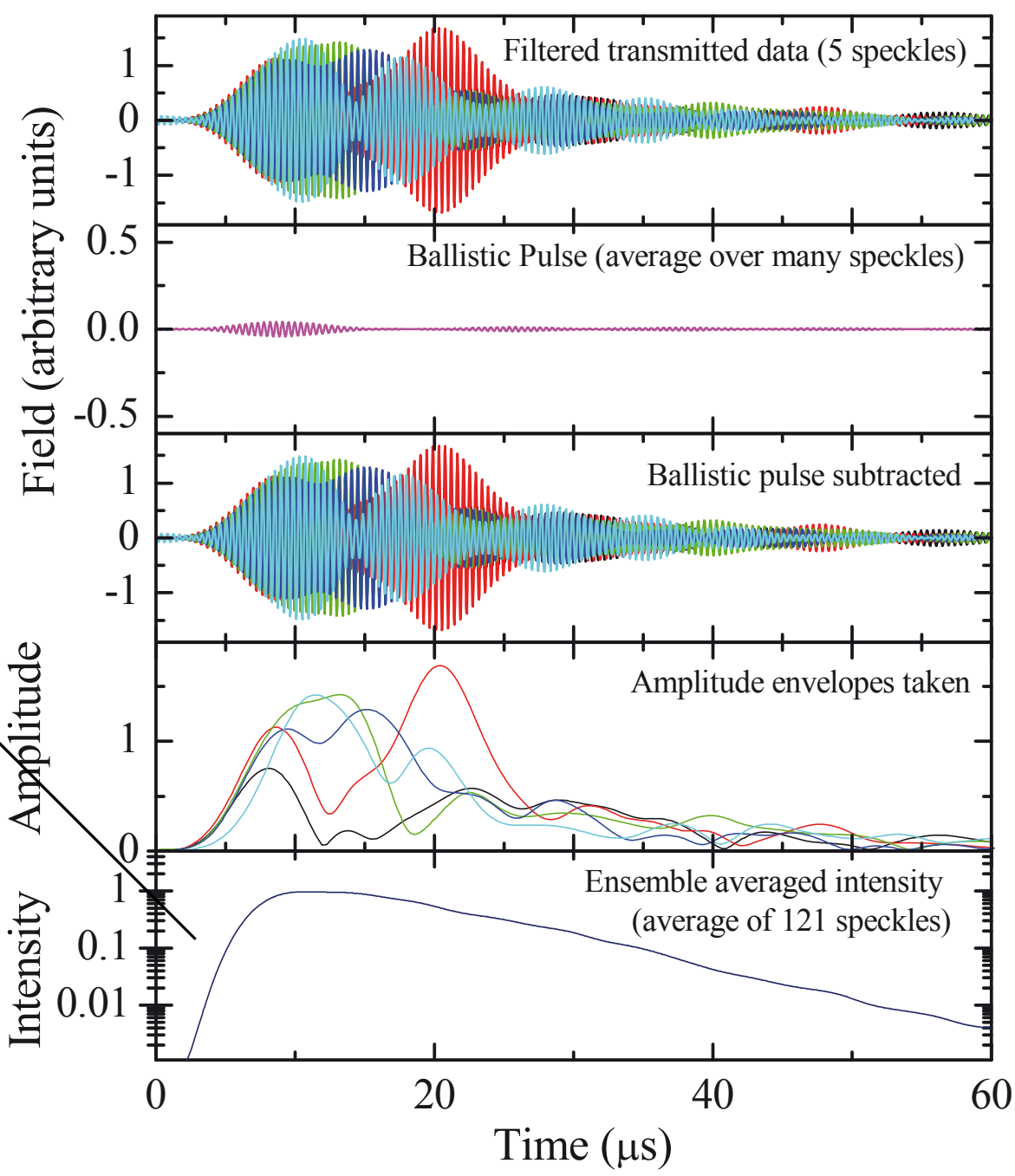


Searching for suitable 3D materials...

An acoustic 3D system - suspensions of glass spheres in a liquid:

- Diffusion approximation gives an excellent description of energy transport by multiply scattered ultrasonic waves $(L / \ell>3)$.

- Unified physical picture of energy transport by ballistic and diffusive waves was developed based on effective medium SpFA model. $\left(v_{\mathrm{e}} \sim v_{\mathrm{g}}\right)$

- But....

Scattering is not so strong, despite large impedance contrast.

$$
3<k \ell<10 \text { (for } 2<k_{w} a<10 \text { ) }
$$

Absorption is not negligible.

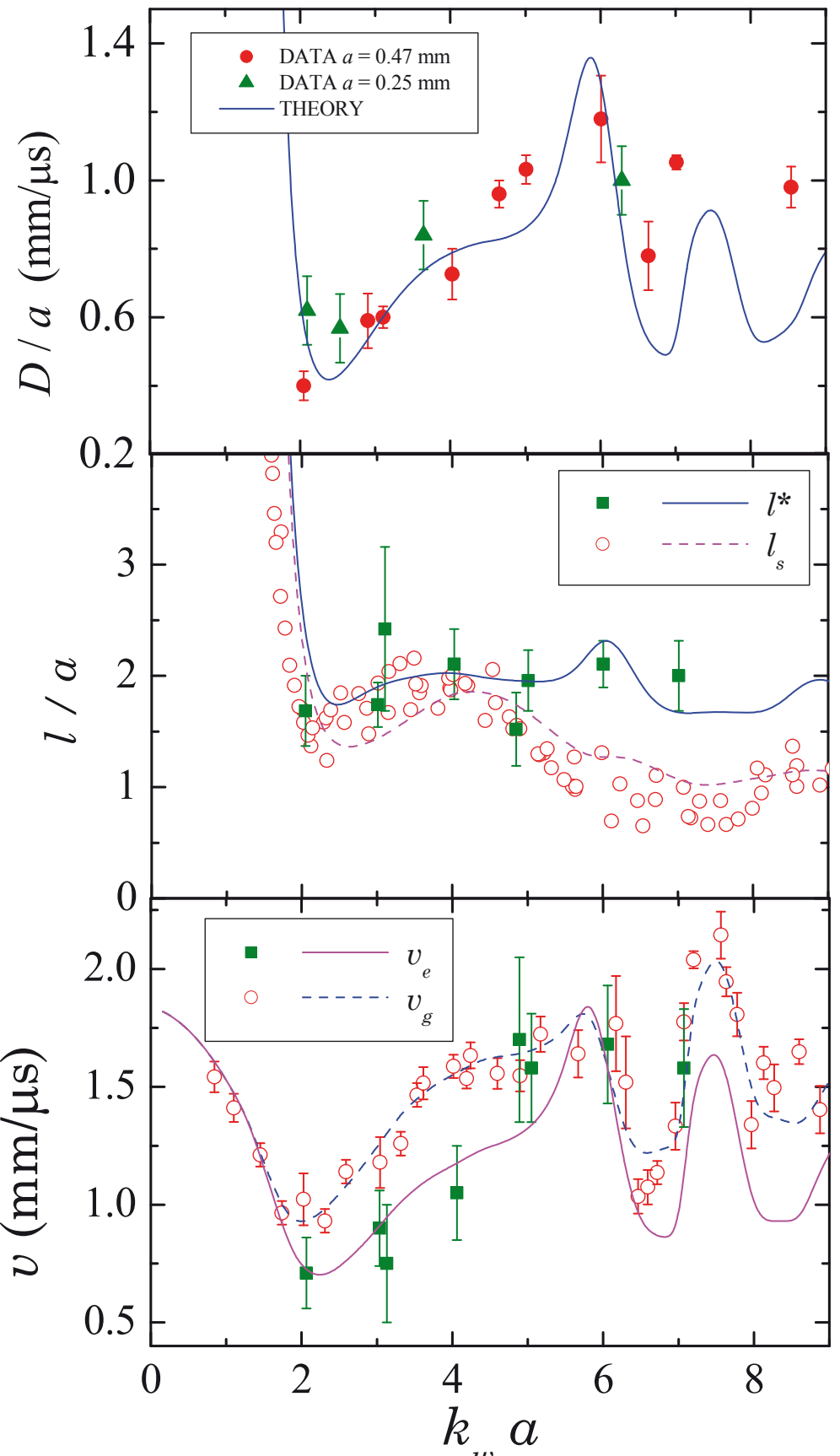

[Schriemer et al., PRL ${ }^{w} 79,3166$ (1997)] 
Searching for suitable 3D materials...

Porous solid 3D materials may be more interesting...

B) a sintered network of glass beads ( $\phi_{\text {glass }}=0.3, a_{\text {bead }}=0.05 \mathrm{~mm}$ )

What's different?

- Solid network : elastic waves

$\Rightarrow$ Two polarizations

(both longitudinal and traverse waves).

- Sintered networks are

- analogous to porous rocks

- a random percolation system well above threshold

- mesoscopic model for glasses $\Rightarrow$ "mesoglasses"

- Very strong scattering may be possible, with less absorption

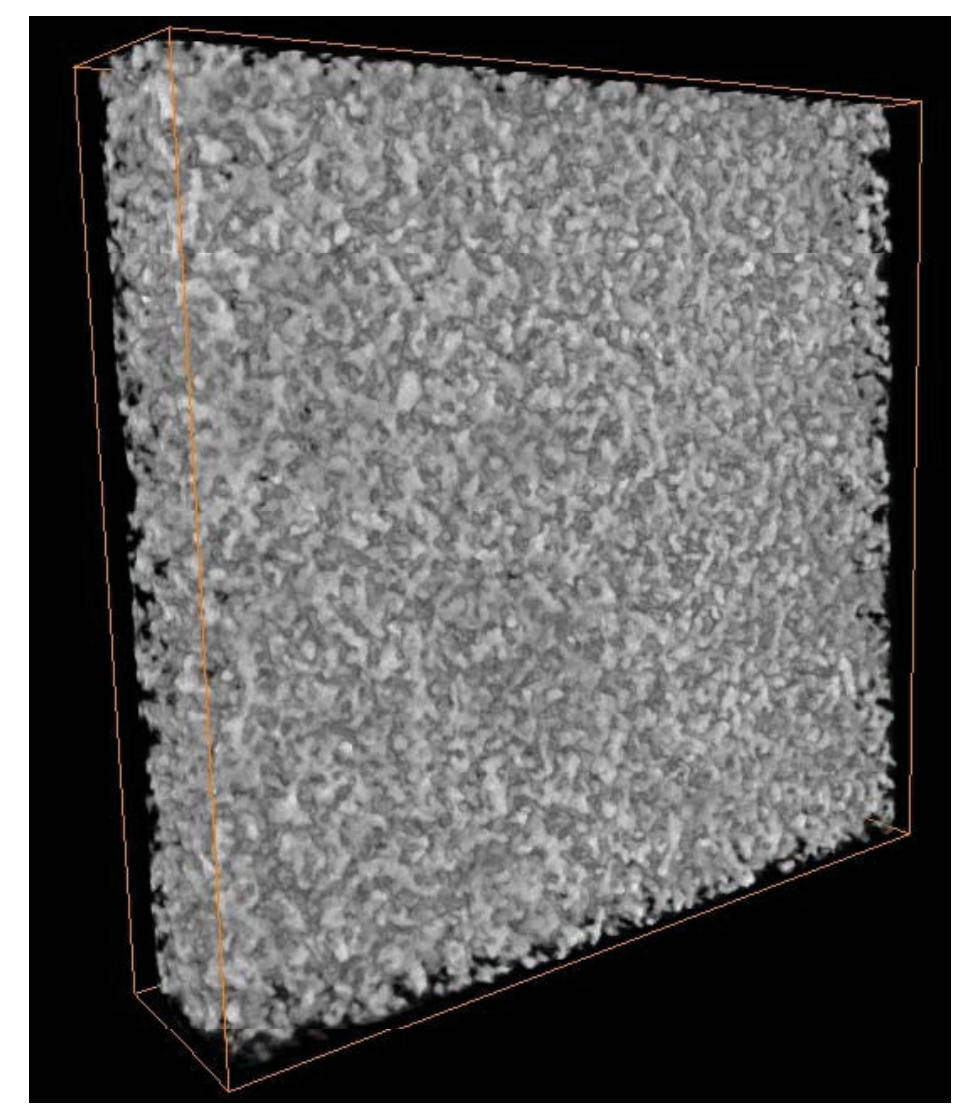




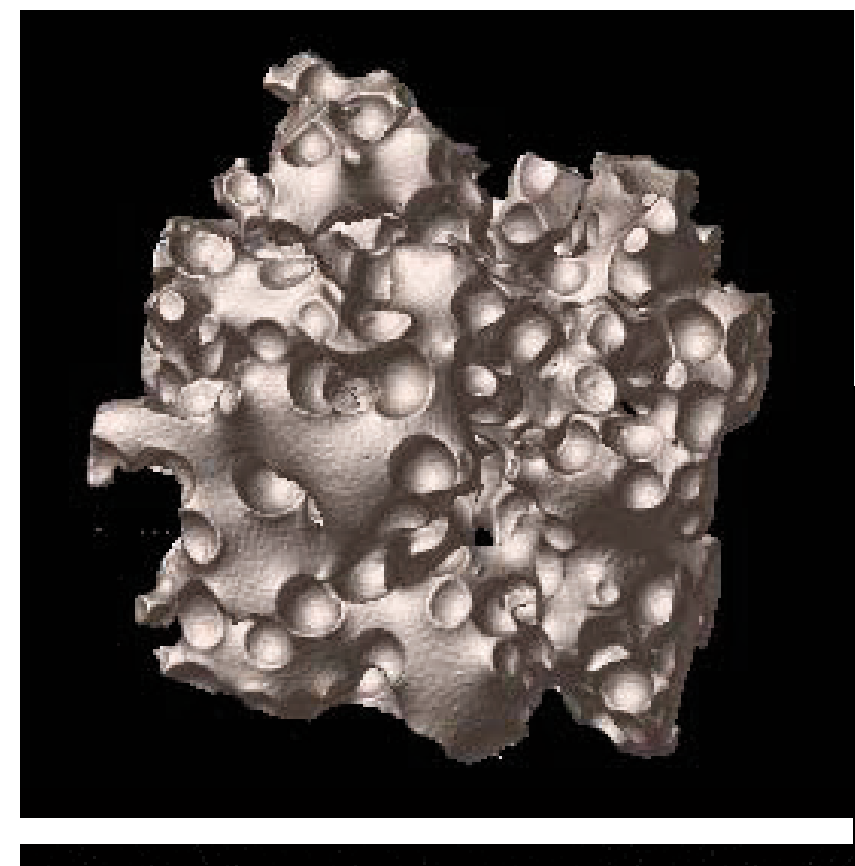

Structure of one of our sintered glass bead "mesoglasses"
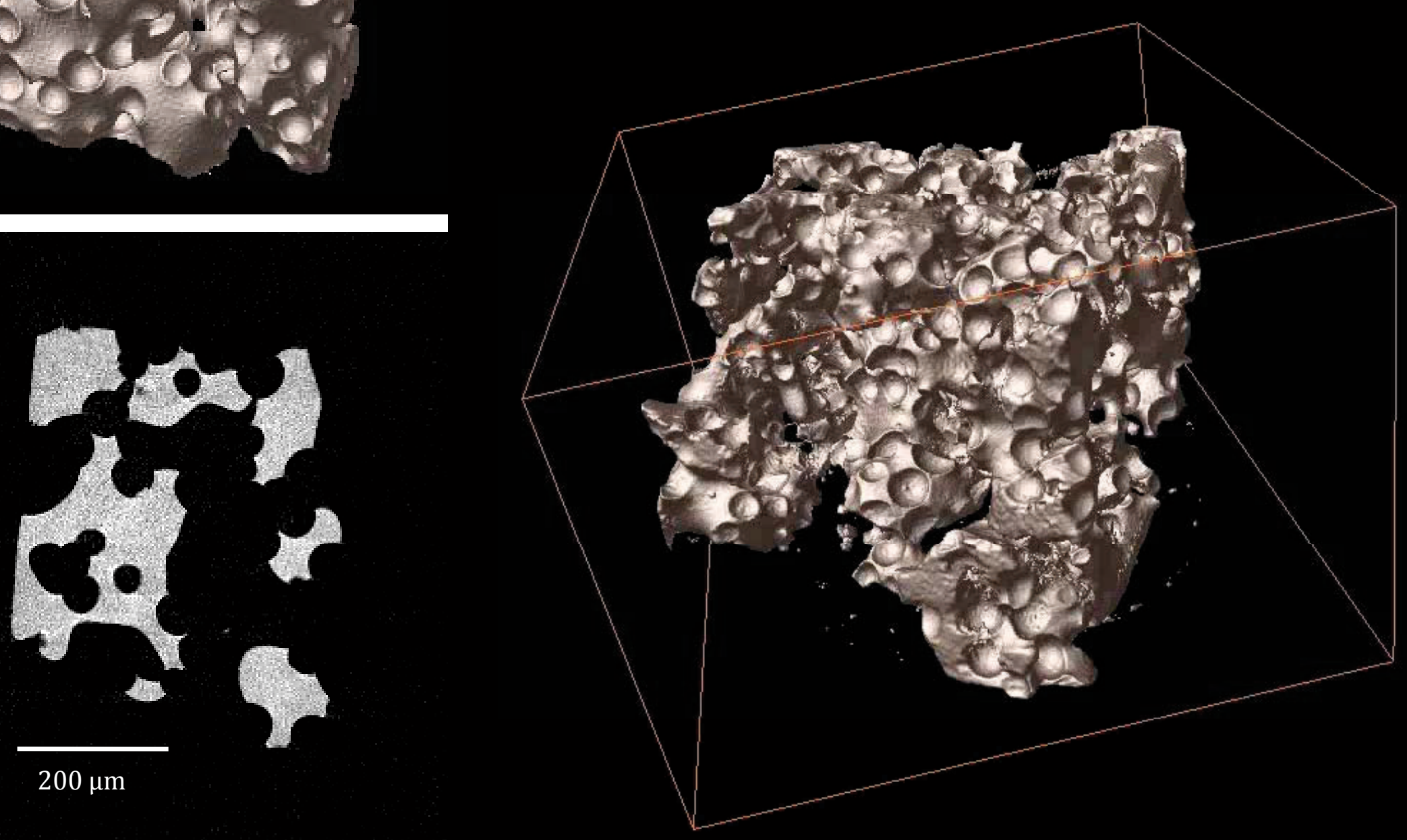
Wave transport in porous elastic glass bead networks

Energy equipartition:

Weaver, JASA 71, 1609 (1982)

Ryzhik et al., Wave Motion

24, 327 (1996)

Hennino et al., PRL 86,

3447 (2001)

A single diffusive pulse is observed, since the longitudinal and transverse polarizations mix at each scattering.

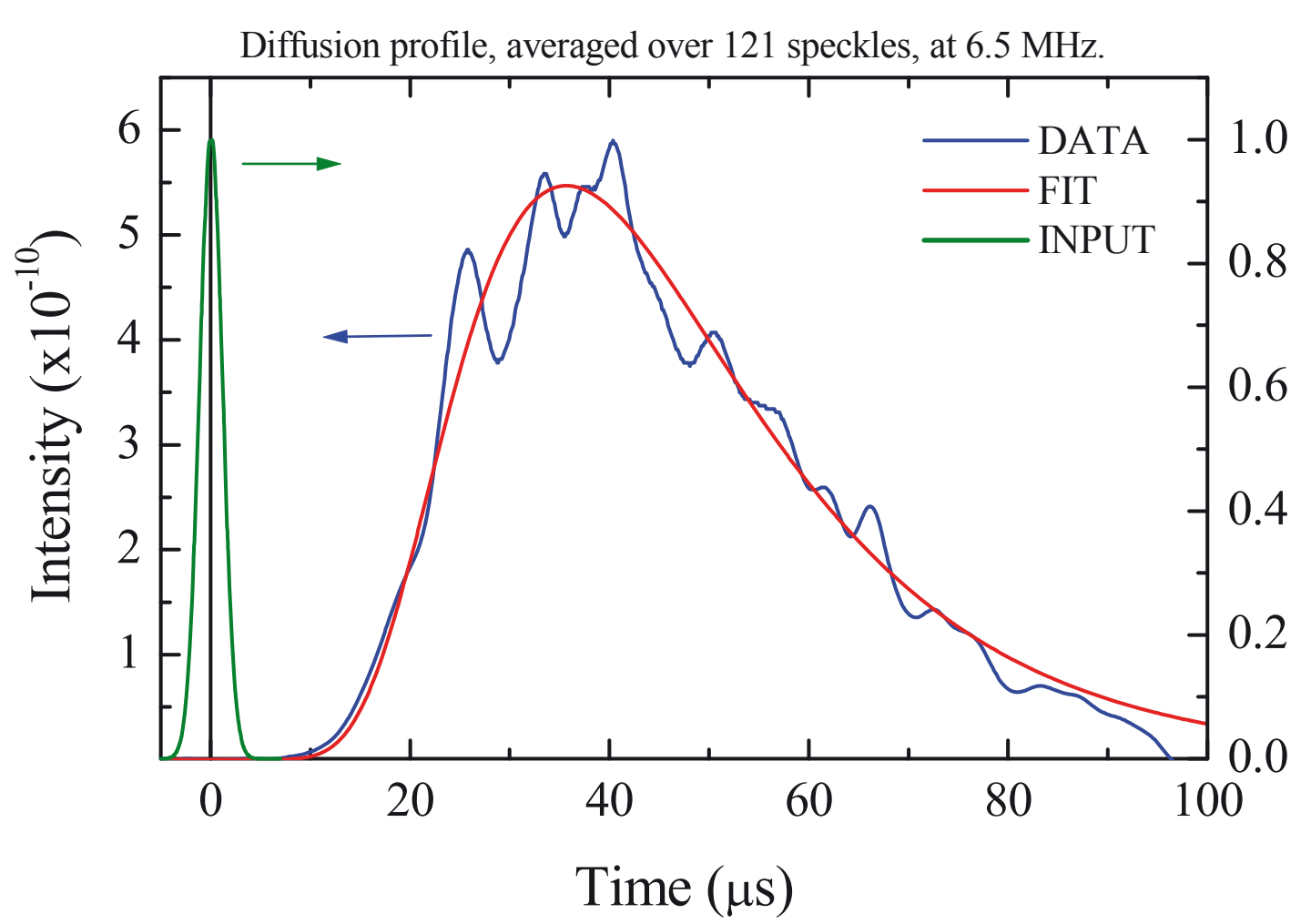

The effective $D$ is determined by the energy-density weighted average of the two polarizations. In the simplest case, we have

Longitudinal energy density:

$U_{L}(\omega) \propto \rho_{L}(\omega)=\frac{k^{2}}{2 \pi^{2}} \frac{d k}{d \omega} \propto \frac{\omega^{2}}{v_{p, L}^{2} v_{g, L}}$
Transverse energy density:

$$
U_{T}(\omega) \propto \frac{2 \omega^{2}}{v_{p, T}^{2} v_{g, T}}
$$


Effective diffusion coefficient

$$
D=\frac{1}{3} \frac{\frac{l_{L}^{*}}{v_{p, L}^{2}}+\frac{2 l_{T}^{*}}{v_{p, T}^{2}}}{\frac{1}{v_{p, L}^{2} v_{g, L}}+\frac{2}{v_{p, T}^{2} v_{g, T}}}=\frac{1}{3} v_{e} l^{*}
$$

with

$$
\begin{aligned}
& l^{*}=\left(\frac{l_{L}^{*}}{v_{p, L}^{2}}+\frac{2 l_{T}^{*}}{v_{p, T}^{2}}\right) /\left(\frac{1}{v_{p, L}^{2}}+\frac{2}{v_{p, T}^{2}}\right) \quad \text { Effective transport mean free path } \\
& v_{e}=\left(\frac{1}{v_{p, L}^{2}}+\frac{2}{v_{p, T}^{2}}\right) /\left(\frac{1}{v_{p, L}^{2} v_{g, L}}+\frac{2}{v_{p, T}^{2} v_{g, T}}\right) \quad \text { Effective energy velocity }
\end{aligned}
$$

Must account for absorption and boundary conditions, as for acoustic waves:

- Absorption - reduces the time resolved intensity by $\exp \left(-t / \tau_{a}\right)$

- Depth $z$ 'inside the sample where diffusion "begins" (location of the diffusive source resulting from the coherent input pulse - still expect: $z^{\prime} \approx l^{*}$

- Realistic boundary conditions - account for reflections of elastic waves at the sample boundaries. 
Diffusive pulse propagation - Transmitted flux for a plane wave pulse: solution of the diffusion equation with boundary conditions for a slab sample. (Carslow and Jaeger, Heat Conduction in Solids (Clarendon Press, Oxford 1990))

$$
J(t)=\frac{2 D \exp \left(-t / \tau_{a}\right)}{L^{2}} \sum_{n=1}^{\infty} A_{n} \exp \left(-D \beta_{n}{ }^{2} t / L^{2}\right) \quad \begin{aligned}
& \text { [e.g. Page et al., Phys. } \\
& \text { Rev. E 52, } 3106 \text { (1995)] }
\end{aligned}
$$

where

$$
A_{n}=\frac{\beta_{n}\left[\beta_{n} K \sin \beta_{n}-\cos \beta_{n}\right]\left[\beta_{n} K \cos \left(\beta_{n} z^{\prime} / L\right)+\sin \left(\beta_{n} z^{\prime} / L\right)\right]}{\beta_{n}^{2} K^{2}+1+2 K}
$$

$\beta_{n}$ are the solutions of the transcendental equation $\tan \beta=\frac{2 \beta K}{\beta^{2} K^{2}-1}$

and $K=z_{0} / L \quad$ Here $z_{0}$ is the extrapolation length,

$$
z_{0} \equiv\left(2 l^{*} / 3\right)[(1+R) /(1-R)]
$$

determined by the angle-averaged reflection coefficient of the equipartitioned flux at the boundaries $R \equiv J_{\text {reflected }} / J_{\text {incident }}$

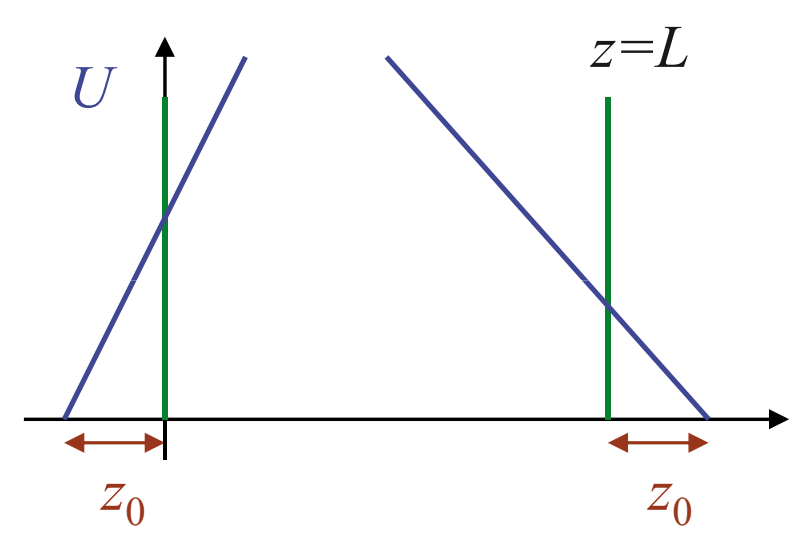


Wave transport in porous elastic glass bead networks

Frequency dependence of $D \quad$ [Page et al., Phys. Status Solidi C, 12925 (2004)]

- $D$ increases rapidly when $f<1 \mathrm{MHz}$

-Plateau in the strong scattering regime!

-Inset shows results of simulations for a percolation model of glasses by Sheng et al. [PRL 72, 234 (1994)]

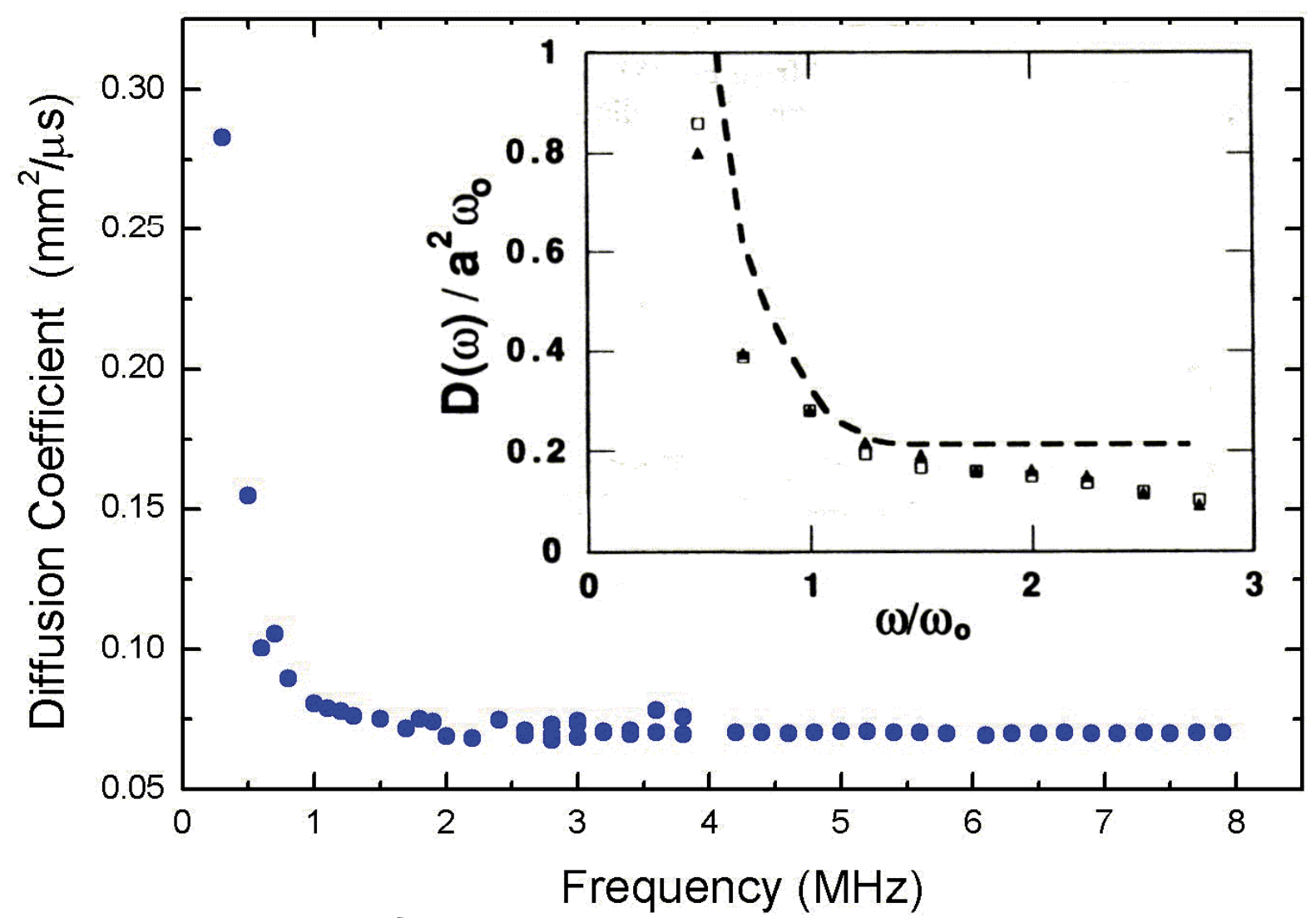

Crossover frequency:

$$
\omega_{\mathrm{o}} \sim \frac{v_{p}}{\xi} \quad \Rightarrow \quad f_{\mathrm{o}} \sim 0.8 \mathrm{MHz}
$$

since the percolation correlation length $\xi \sim 6 a$ at $\phi=0.3$ [Schriemer et al., Waves in Random Media 6, 361 (1996)]

$\Rightarrow$ Onset of the plateau in $D$ gives a measure of the size of the largest pores. 
Glass bead networks - Diffusion coefficient plateau

- Very strong scattering $\left(k l_{s} \sim 1, l^{*}\right.$ $\left.\sim l^{*}{ }_{\min }=2 a\right)$, but the modes are not localized at these frequencies.

$\Rightarrow$ the loffe-Regel criterion

$$
k l_{s} \leq 1
$$

does not necessarily imply localization for acoustic waves [Sheng et al., PRL 72, 234 (1994)]

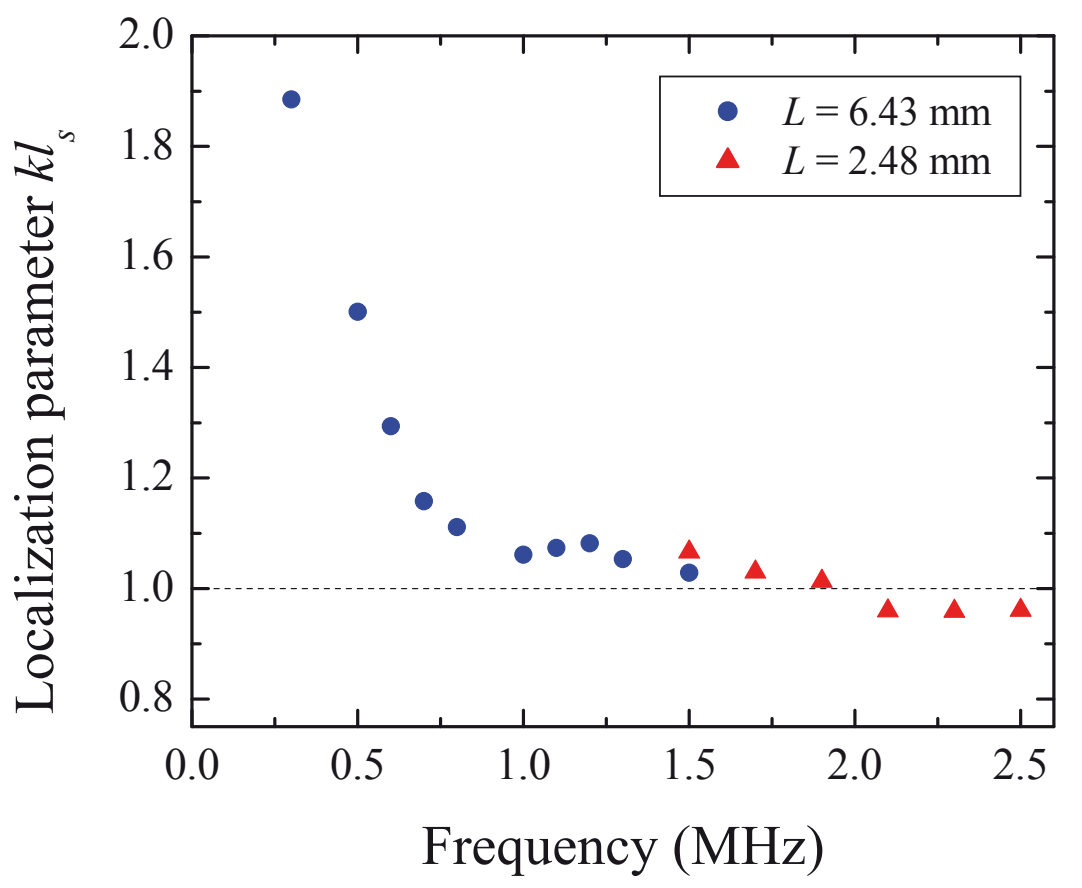

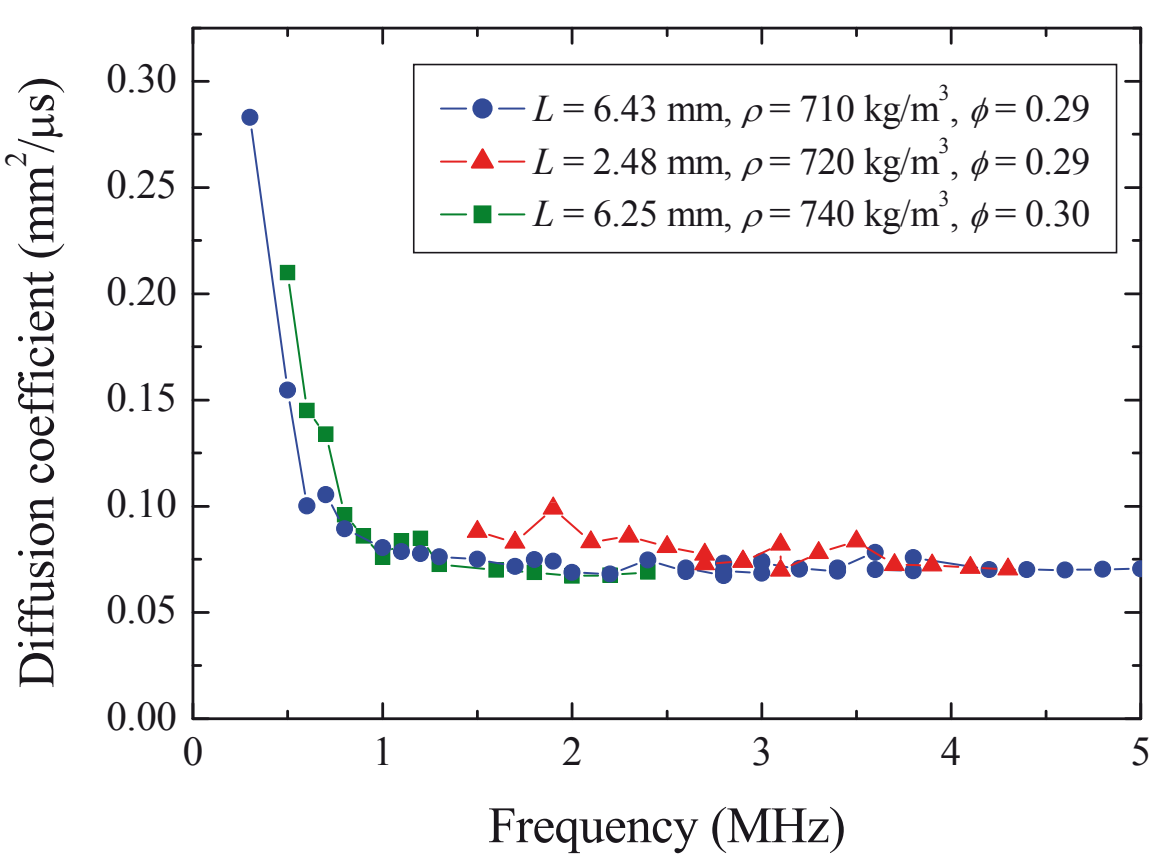

- The plateau in $D$ is a precursor of localization - subdiffusive behaviour.

- Similar behaviour has been predicted for glasses near the thermal conductivity plateau around $20 \mathrm{~K}$ (sinter is a "mesoscopic glass").

- For more, including density of states measurements, see the posters by Laura Cobus and Anatoliy Strybulevych at this School. 
C) Better samples for localization expts: Mesoglasses made from aluminum beads

- Larger particles $\Rightarrow$ can access higher effective frequencies

- Less intrinsic absorption.

Aluminum volume fraction: $\quad \phi=0.55$ Monodisperse beads:

radius, $a_{\text {bead }}=2.05 \mathrm{~mm}$

Sample width >> thickness ( $L: 8$ to $23 \mathrm{~mm}$ )

Pulsed ultrasonic transmission measurements (waterproofed samples, in a water tank)

Frequency range: 0.1 to $3 \mathrm{MHz}(6 \geq \lambda / a \geq 1)$
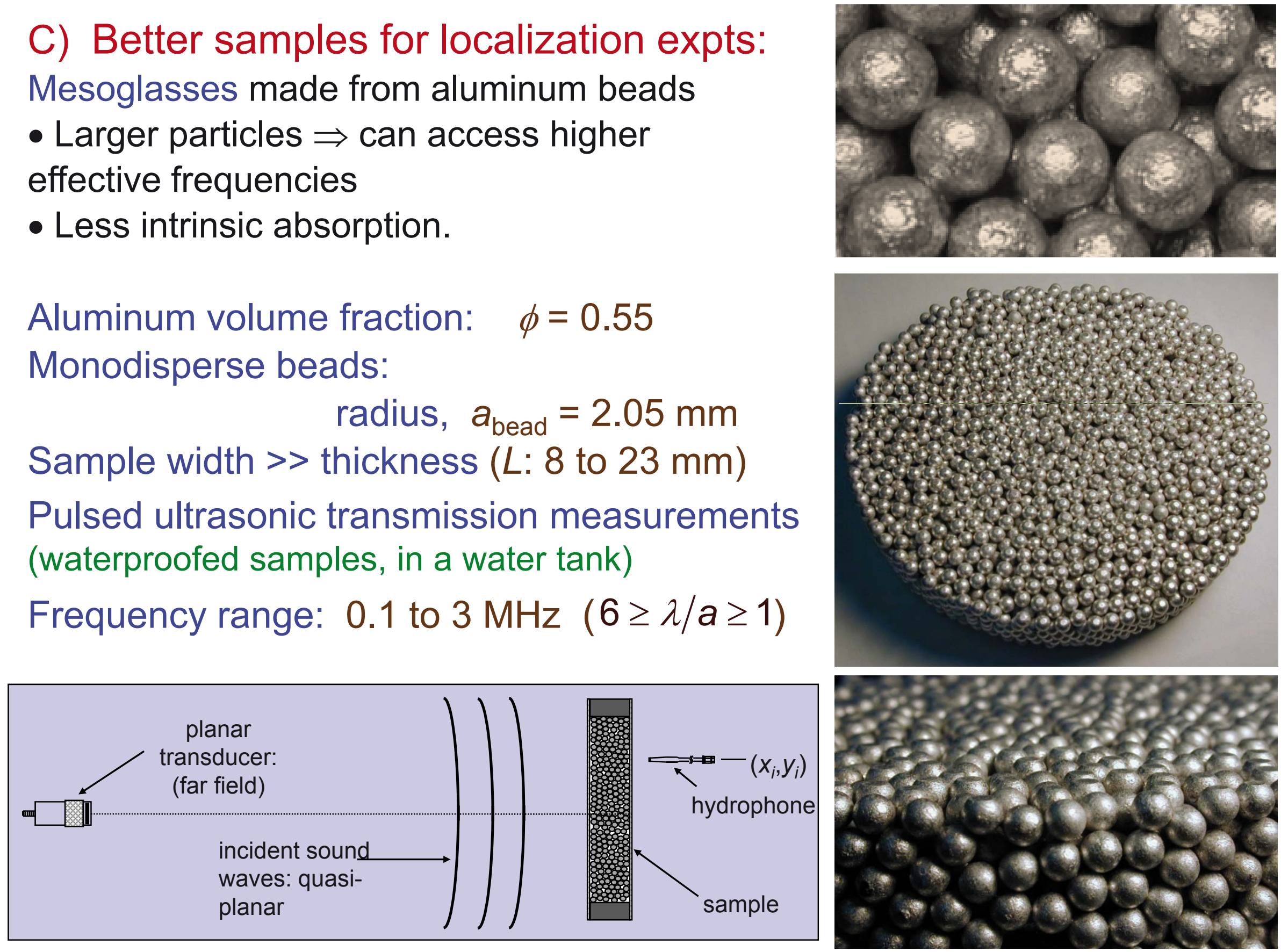
Coherent transport in disordered Al mesostructures:

Ballistic transport: Average the transmitted field to recover the weak coherent pulse and measure :

- phase velocity: $v_{p}=\omega / k$

- group velocity: $v_{g}=\mathrm{d} \omega / \mathrm{d} k$

- scattering mean free path, $\ell: I=I_{0} \exp [-L / \ell]$

Amplitude transmission coefficient:

Bandgaps arise from weakly coupled resonances of the aluminum beads (Turner \& Weaver, 1998)
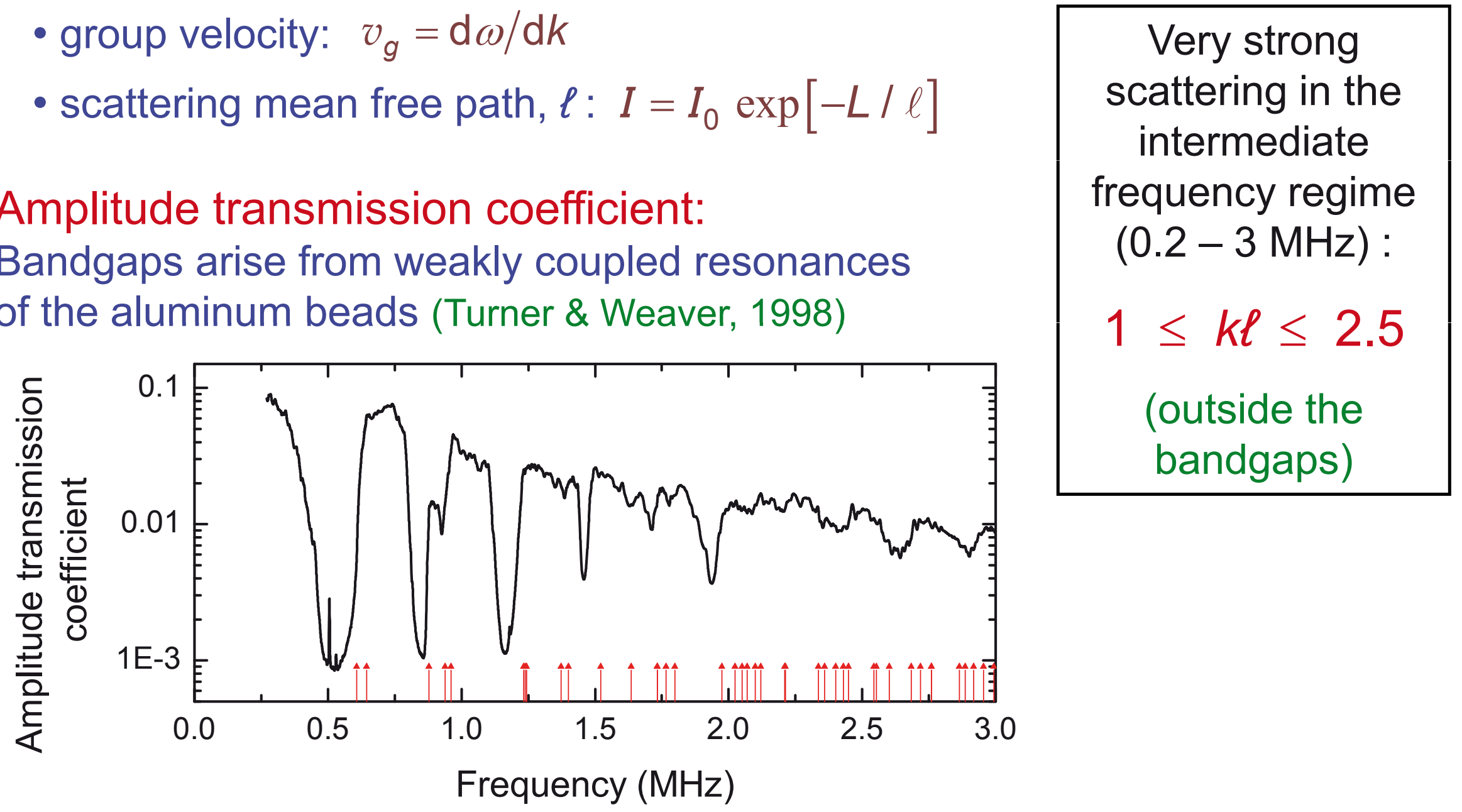


\section{Time-dependent transmission, $I(t)$.}

- Measure multiply scattered field in many independent speckles by scanning the hydrophone.

- Digitally filter the field to limit bandwidth ( $5 \%$ usually)

- Determine $I(t)$ by averaging the squared transmitted pulse envelopes. (Normalize by the peak of the input pulse)

- First compare with the diffusion model, using realistic boundary conditions (e.g. see Page et al., Phys. Rev. E 52, 3106 (1995) for ultrasonic waves)

$\left[z_{0}\right.$ - extrapolation length; $z^{\prime}$ - penetration depth; $\tau_{a}$ - absorption time]

- For elastic media, the diffusion coefficient $D_{B}=1 / 3 v_{E} \ell^{*}$ is the energydensity weighted average of longitudinal and transverse waves.
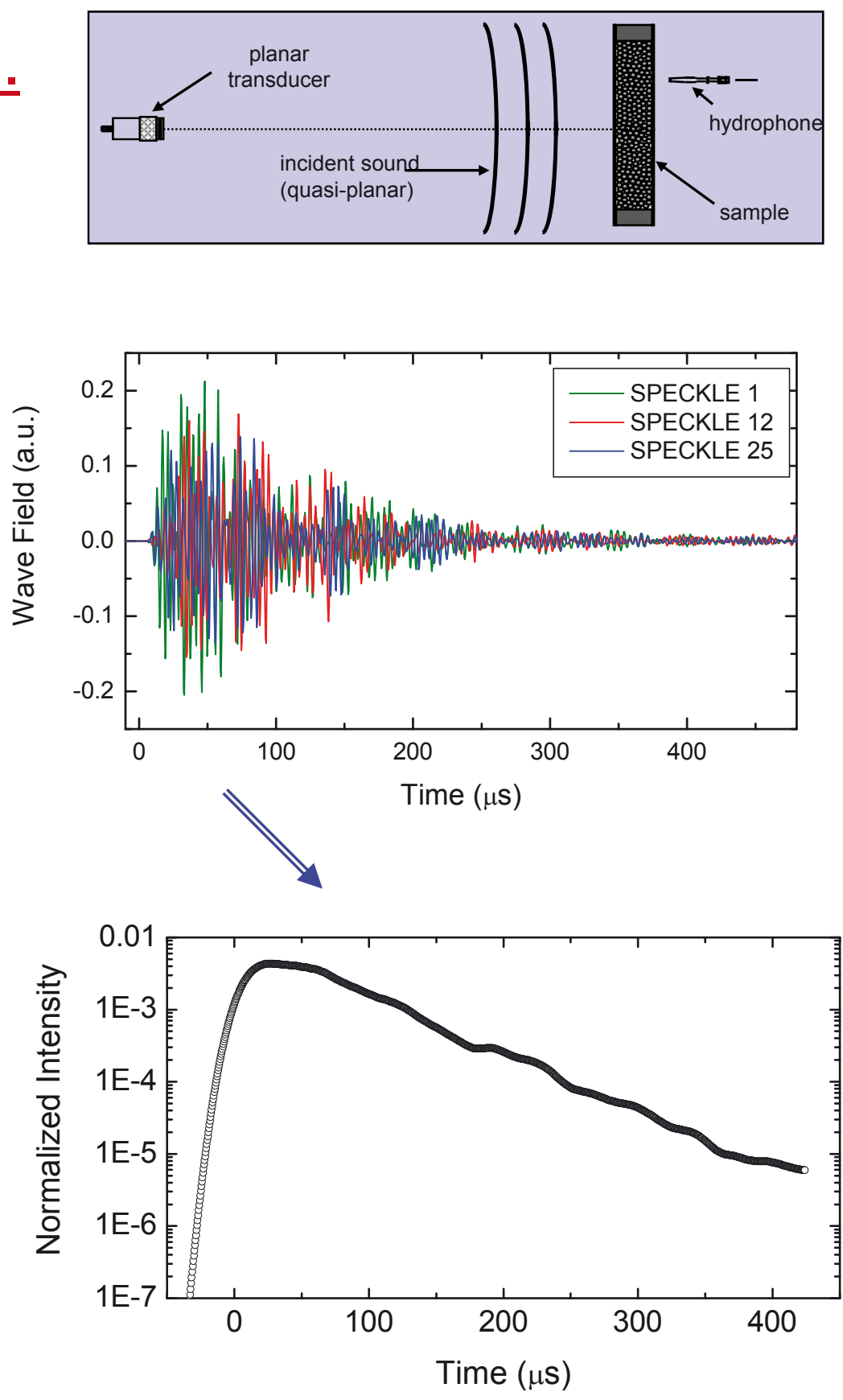
Time-dependent transmission at low frequencies:

(below the lowest band gap)

Good fit to the predictions of the diffusion approximation for a plane wave source $\Rightarrow$ measure $D$. (Absorption is too small to measure.)

$f=0.2 \mathrm{MHz}$ :

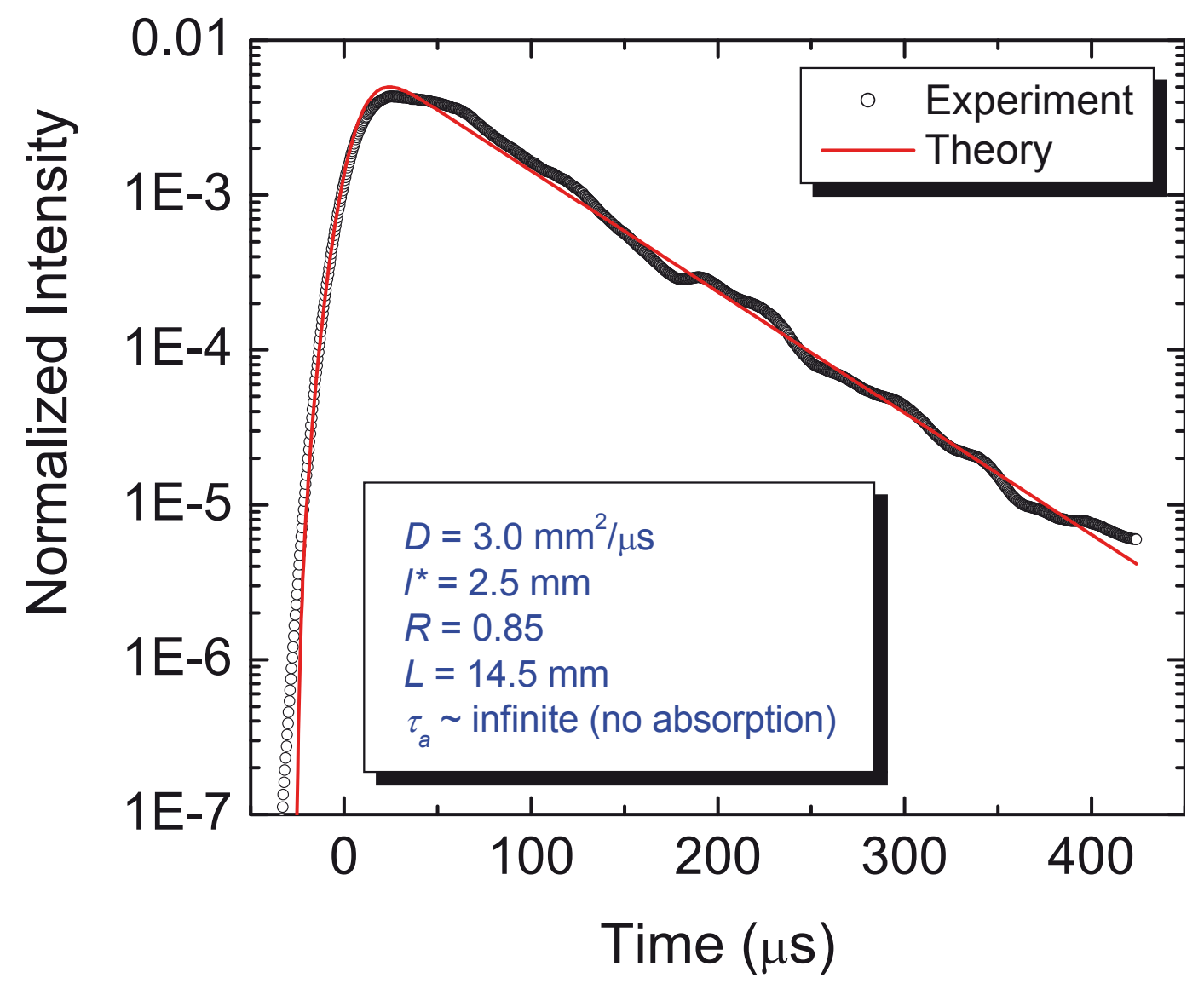

$I(t)$ decays exponentially at long times

$I(t) \sim \exp \left[-t / \tau_{D}\right]$

with

$\tau_{D}=\left(L+z_{0}\right)^{2} / \pi^{2} D_{B}$

Normal diffusive behaviour 
$I(t)$ at higher frequencies (e.g. 2.4 MHz)

Find non-exponential decay of $I(t)$ at long times $\left(t>>\tau_{D}\right) \Rightarrow$ Looks like a diffusion process with $D(t)$ decreasing with propagation time.

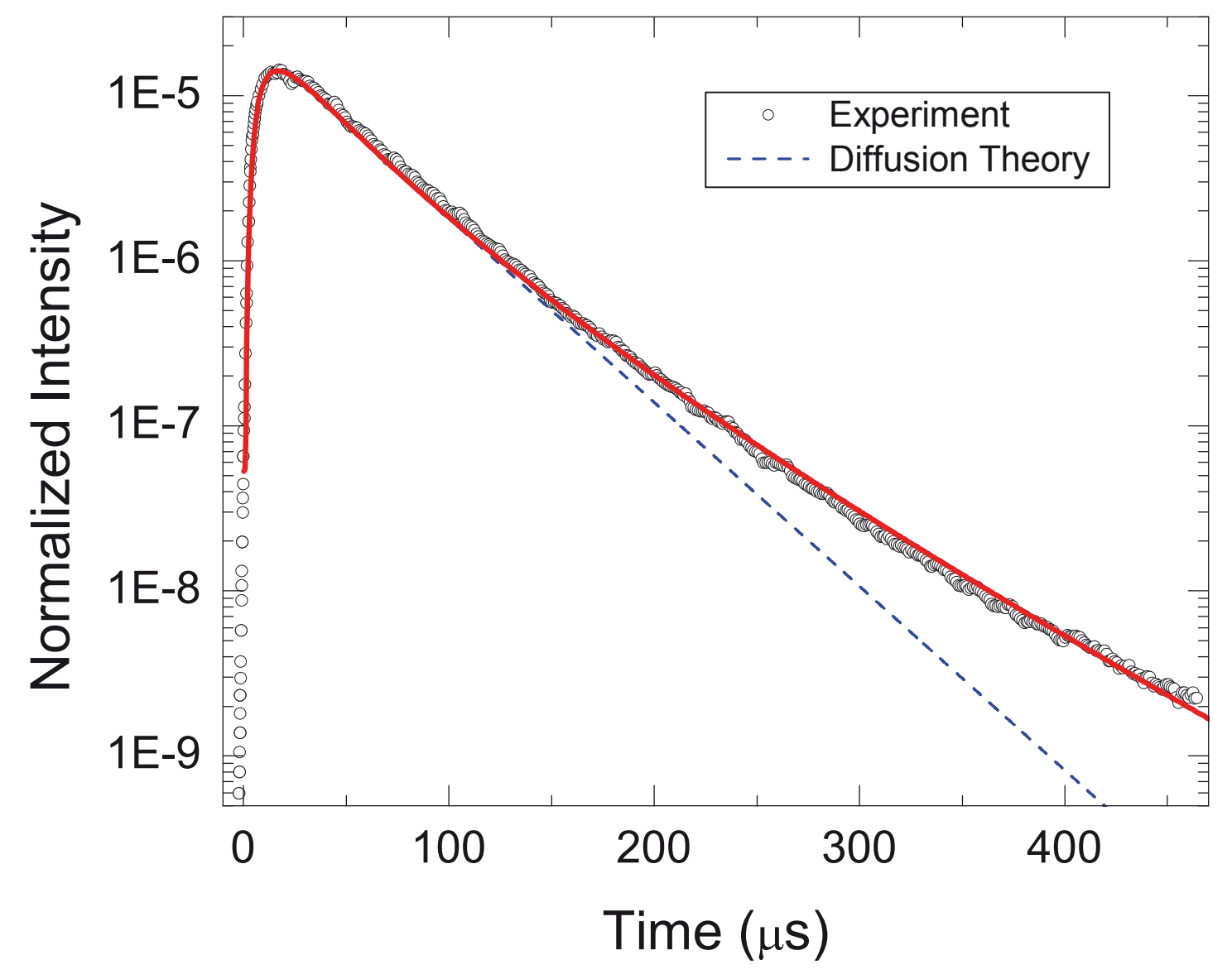

Suggests that sound may be localized 
Quantitative analysis of $I(t)$ at high frequencies $(2.4 \mathrm{MHz})$

- fit the (plane wave) data directly with the recently improved selfconsistent theory of localization [Skipetrov \& van Tiggelen (2006)]

\section{Basic idea:}

The presence of loops increases the return probability as compared to 'normal' diffusion

Diffusion slows down

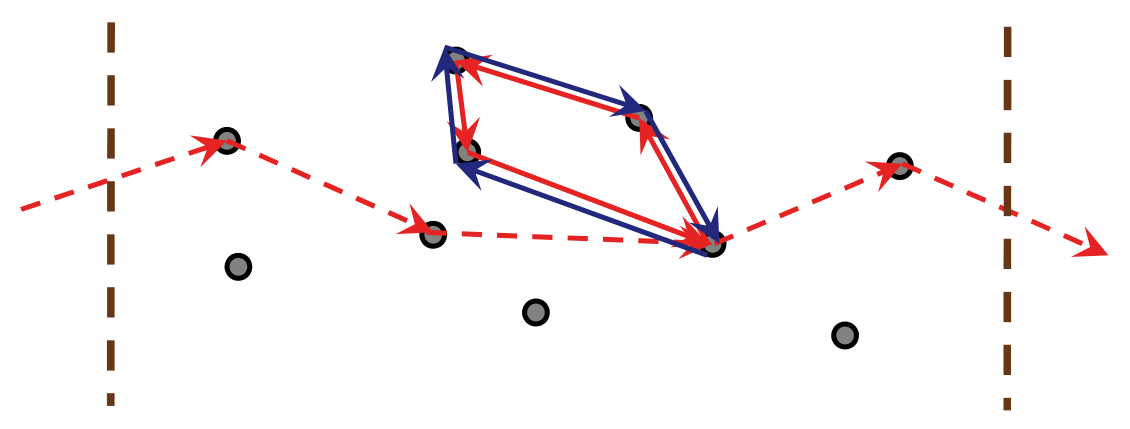

Diffusion constant should be renormalized

$$
D_{\mathrm{B}} \rightarrow D<D_{\mathrm{B}}
$$

Generalization to Open Media:

Loops are less probable near the boundaries

Slowing down of diffusion is spatially heterogeneous

Diffusion constant becomes position-dependent

$$
D_{\mathrm{B}} \rightarrow D(\mathbf{r})<D_{\mathrm{B}}
$$


Quantitative analysis of $I(t)$ at high frequencies $(2.4 \mathrm{MHz})$

- fit the (plane wave) data directly with the recently improved selfconsistent theory of localization [Skipetrov \& van Tiggelen (2006)]

Mathematical formulation:

Diffusion equation

$[-i \Omega-\nabla \cdot D(r, \Omega) \nabla] G\left(r, r^{\prime}, \Omega\right)=\delta\left(r-r^{\prime}\right) !$

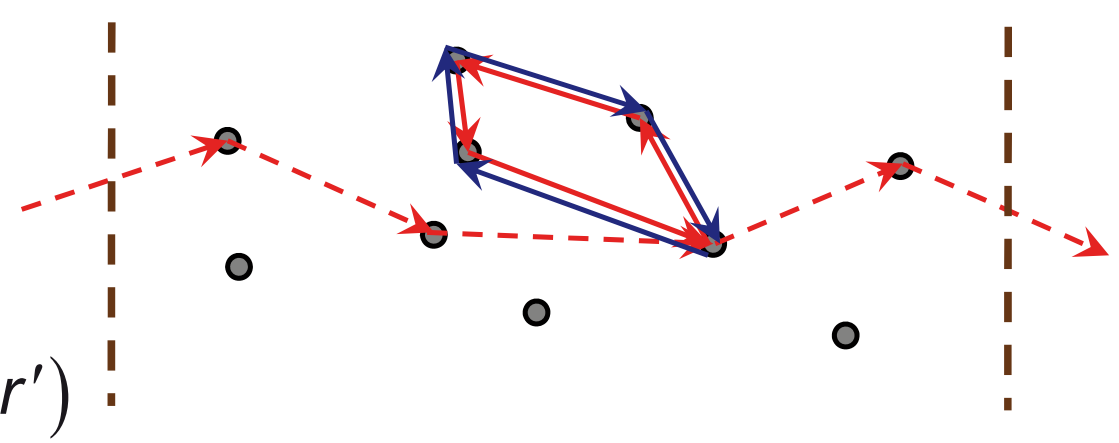

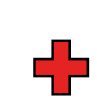

$\left(G\left(r, r^{\prime}, \Omega\right)\right.$ - Intensity Green's function)

Self-consistent equation for the diffusion coefficient

$$
\frac{1}{D(r, \Omega)}=\frac{1}{D_{B}}+\frac{3}{\pi \rho(\omega) D_{B}} G\left(r, r^{\prime}=r, \Omega\right)
$$

$$
(\rho(\omega) \text { - density of states })
$$

Boundary conditions

$$
G\left(r, r^{\prime}, \Omega\right)-z_{0} \frac{D(r, \Omega)}{D_{B}}\left(\mathbf{n} \cdot \nabla G\left(r, r^{\prime}, \Omega\right)\right)=0
$$

Diffusion coefficient depends on position $\mathbf{r}$ and frequency $\Omega$ 
Quantitative analysis of $I(t)$ at high frequencies $(2.4 \mathrm{MHz})$ - fit the (plane wave) data directly with predictions of the self consistent theory of localization for $D(r, \Omega)$ [Skipetrov \& van Tiggelen (2006)]
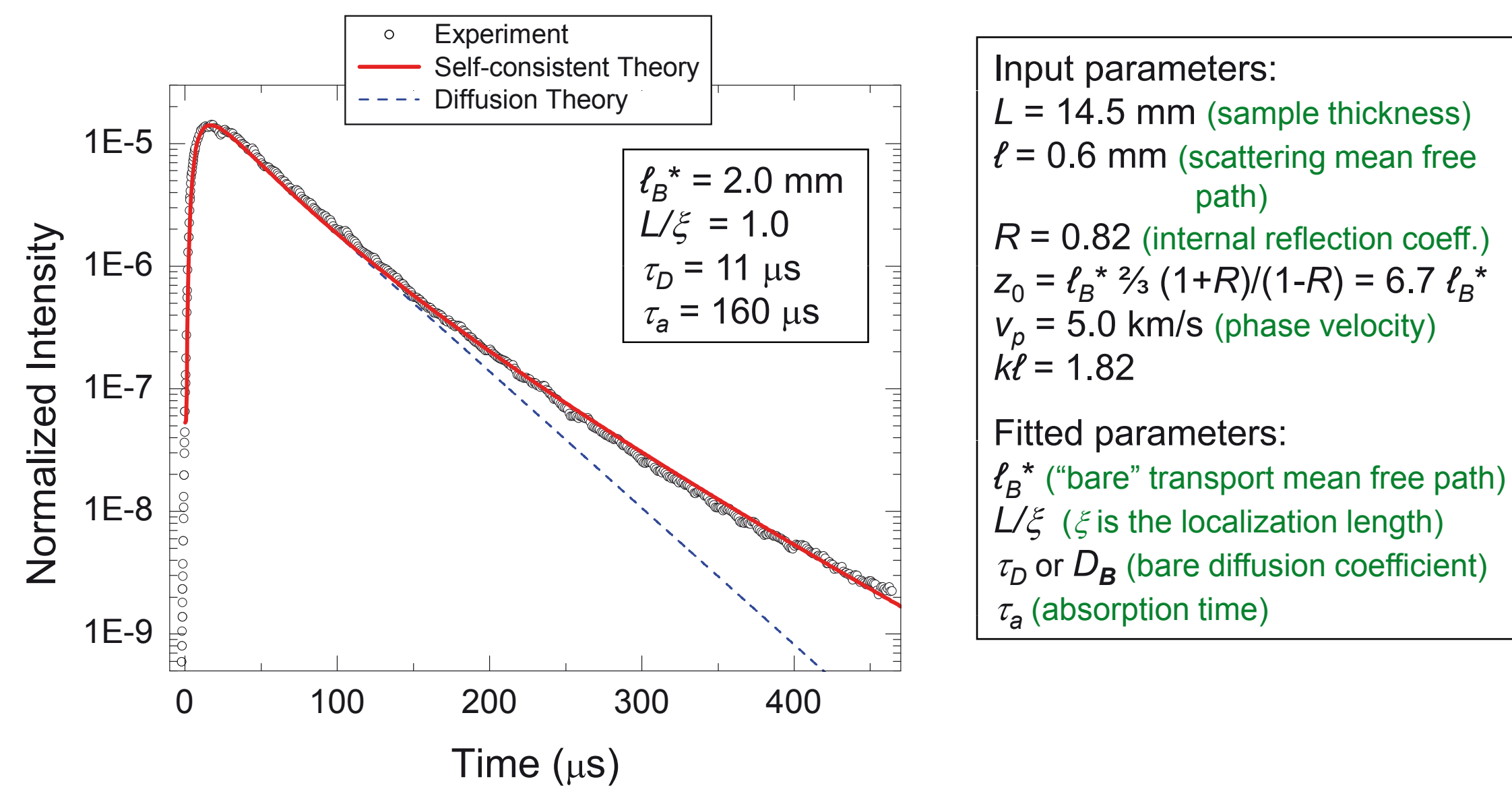

Excellent fit at all propagation times. 
Quantitative analysis of $I(t)$ at high frequencies $(2.4 \mathrm{MHz})$ - fit the (plane wave) data directly with predictions of the self consistent theory of localization for $D(\boldsymbol{r}, \Omega)$ [Skipetrov \& van Tiggelen (2006)]

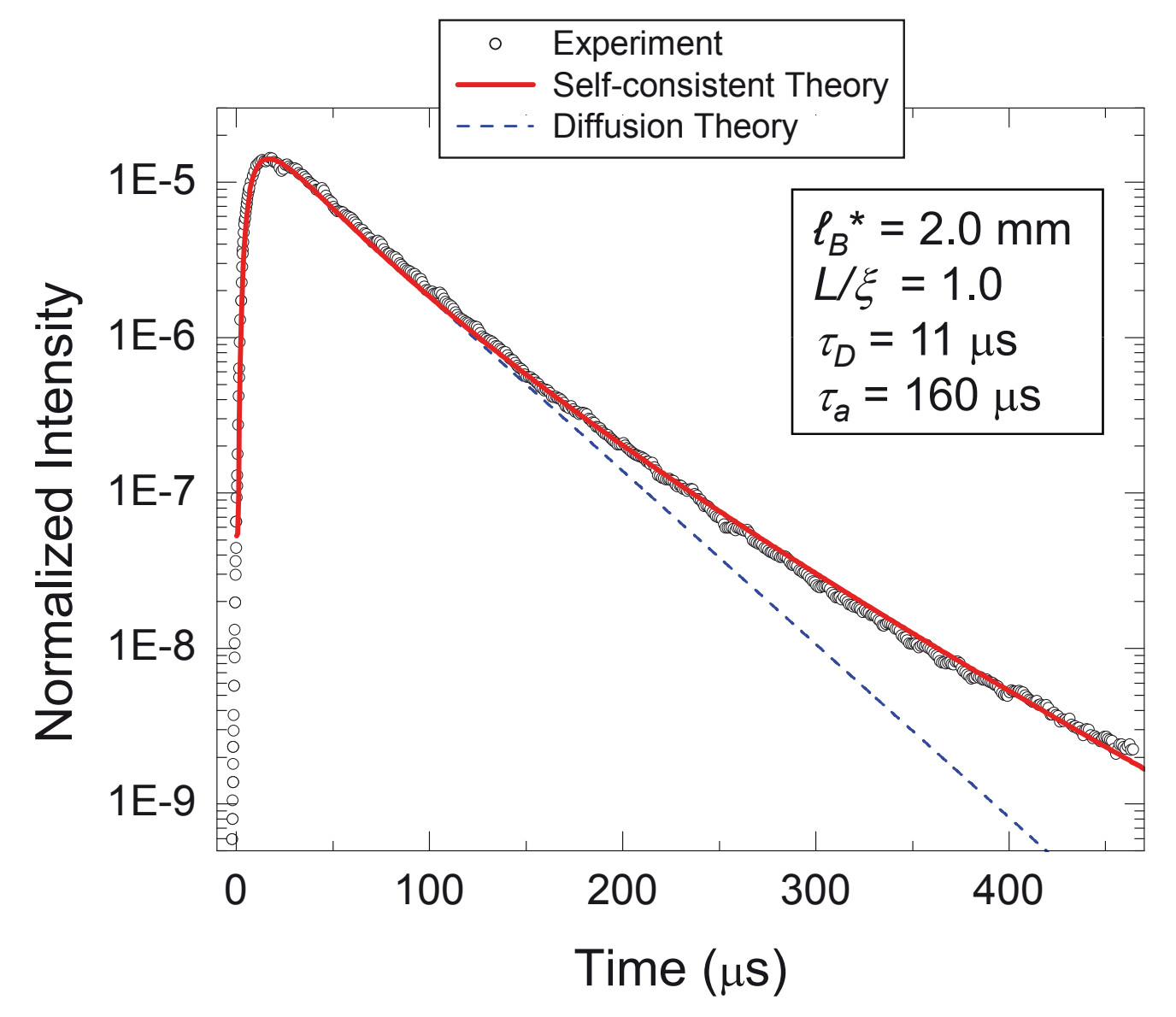

Localization length $\xi$ :

$$
\begin{aligned}
& \frac{\xi}{\ell_{B}{ }^{*}}=\left[\frac{6}{\left(k \ell_{B}{ }^{*}\right)_{c}^{2}}\right] \frac{\chi^{2}}{1-\chi^{4}} \\
& \text { where } \chi=k \ell /(k \ell)_{c} \\
& \text { Localization regime: } \\
& \quad \xi>0, \quad k \ell<(k \ell)_{c}
\end{aligned}
$$

Diffuse regime:

$$
\xi<0, \quad k \ell>(k \ell)_{\mathrm{c}}
$$

Excellent fit at all propagation times with $\xi>0 \quad(L>\xi>L / 4)$

$\Rightarrow$ Strong (but indirect) evidence for the localization of sound 
Self consistent theory of localization predicts a strong and rapid renormalization of $D$ in our samples:

$\frac{1}{D(\Omega, r)}=\frac{1}{D_{B}}+\beta G\left(r, r^{\prime}=r, \Omega\right)$
where $\beta=3 / \pi \rho(\omega) D_{B}$,
$\rho(\omega)=$ D.O.S.

$\Rightarrow D_{B}$ cannot be measured directly, even for $t<\tau_{D}$. $\left(D(\Omega, z) / D_{B}<<1\right.$ for all accessible $t$ ).

$\Rightarrow$ Best fits have: surprising large $D_{B}$ (and hence large $v_{E}$ )

Question: Can this be explained by a reduced density of states (D.O.S.)? - see poster by Eric Lee
Typical curves $\mathrm{D}(\Omega=-i \alpha+\varepsilon, z)$
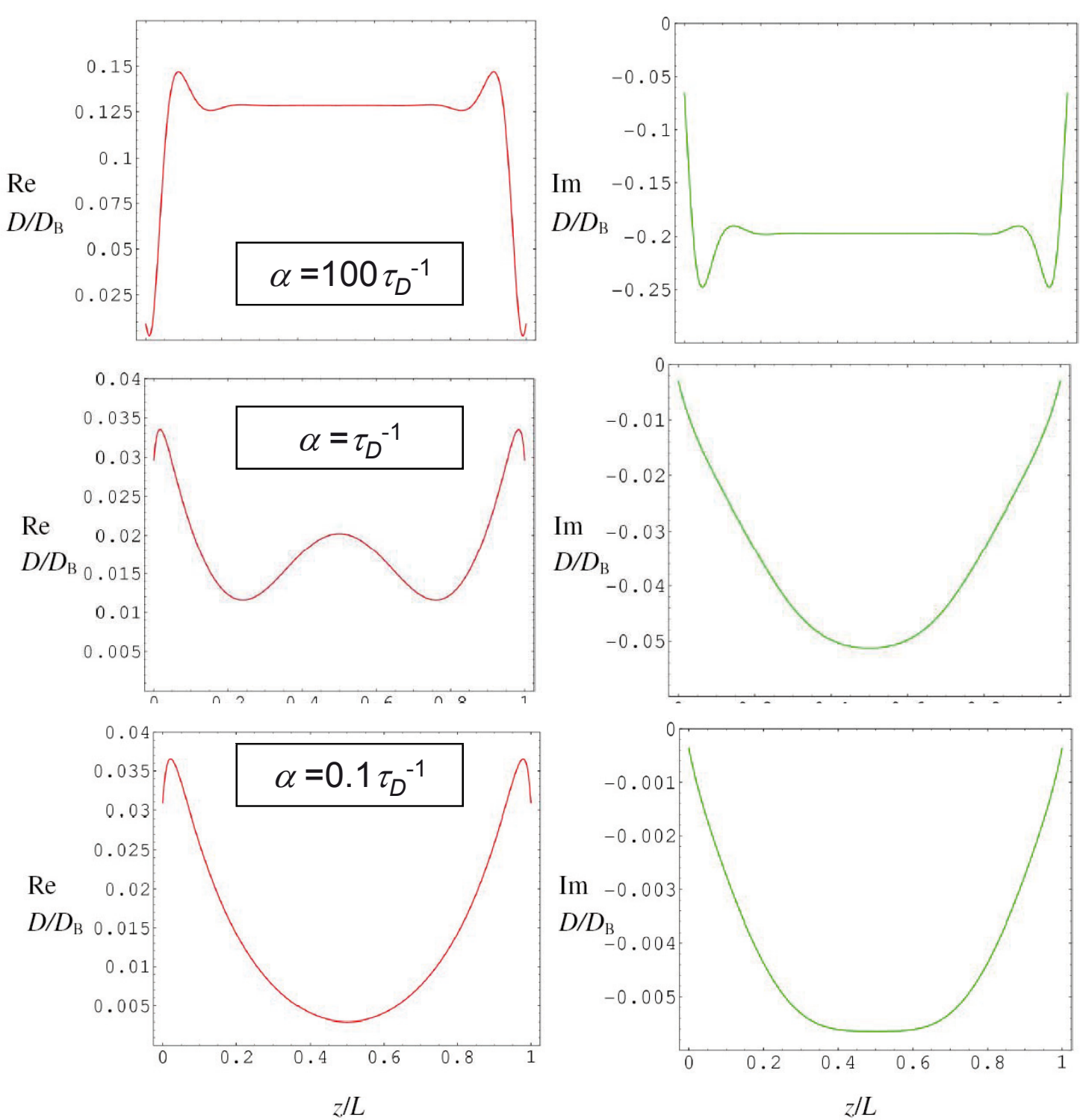


\section{Density of states - see poster by Eric Lee}

- Unusual behaviour below the first bandgap ( constant).

- For $f>0.6 \mathrm{MHz}$, average DOS is consistent with standard predictions:

Debye:

$$
\frac{D(f)}{V}=\phi \frac{12 \pi f^{2}}{\bar{V}^{3}}
$$

Weyl (includes surface

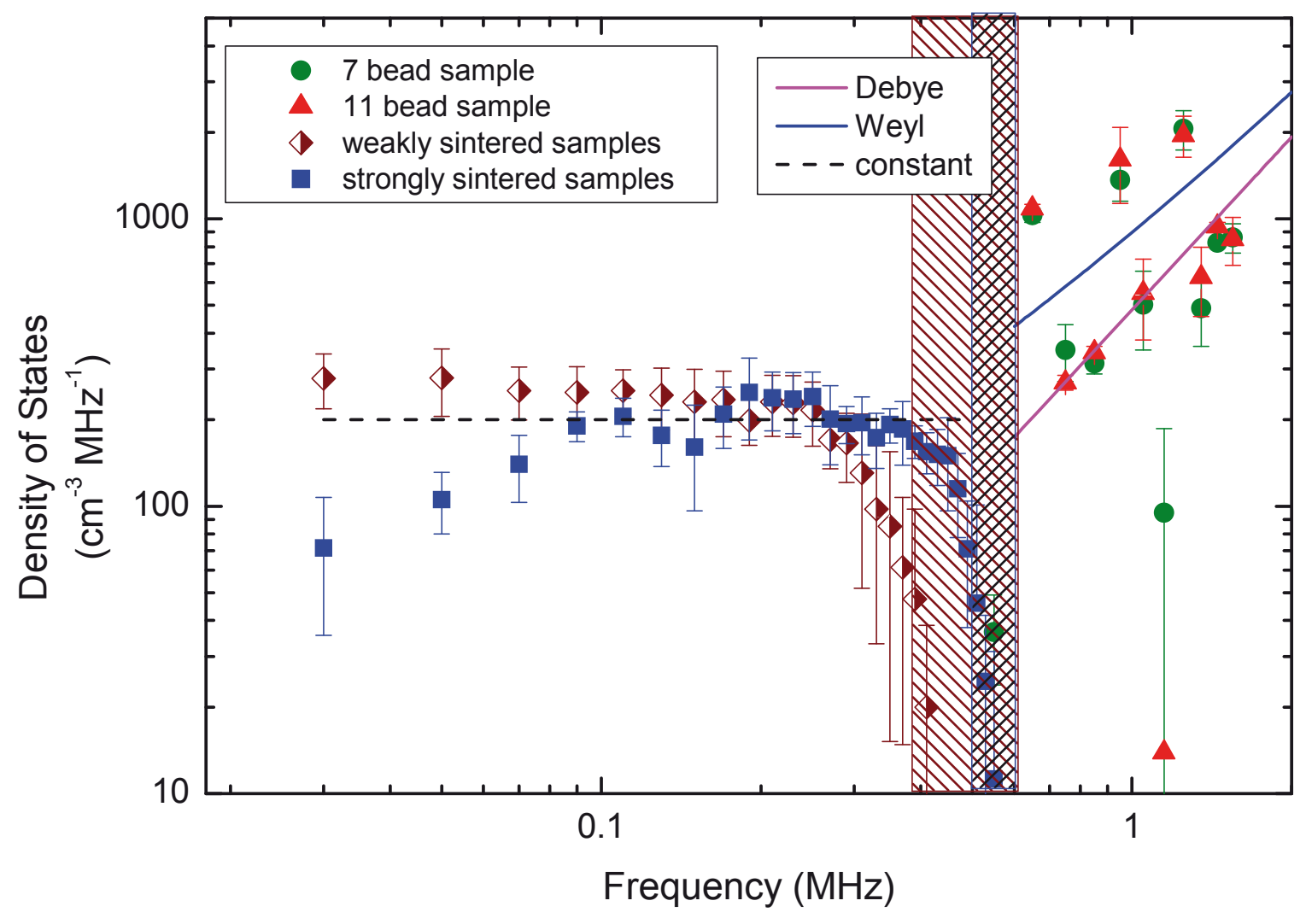
modes)

$$
D(f)=n\left\{\frac{12 \pi v_{\text {sphere }}}{\bar{v}^{3}} f^{2}+\frac{\pi S_{\text {sphere }}}{2} \frac{2-3\left(v_{L} / v_{T}\right)^{2}+3\left(v_{L} / v_{T}\right)^{4}}{v_{L}^{2}\left[\left(v_{L} / v_{T}\right)^{2}-1\right]} f\right\}
$$

Conclude: Large values found for $v_{E}$ near $2 \mathrm{MHz}$ cannot be explained by anomalously low DOS (due to short range correlations or bandgap effects) 
Quantitative analysis of $I(t)$ at high frequencies $(2.4 \mathrm{MHz})$

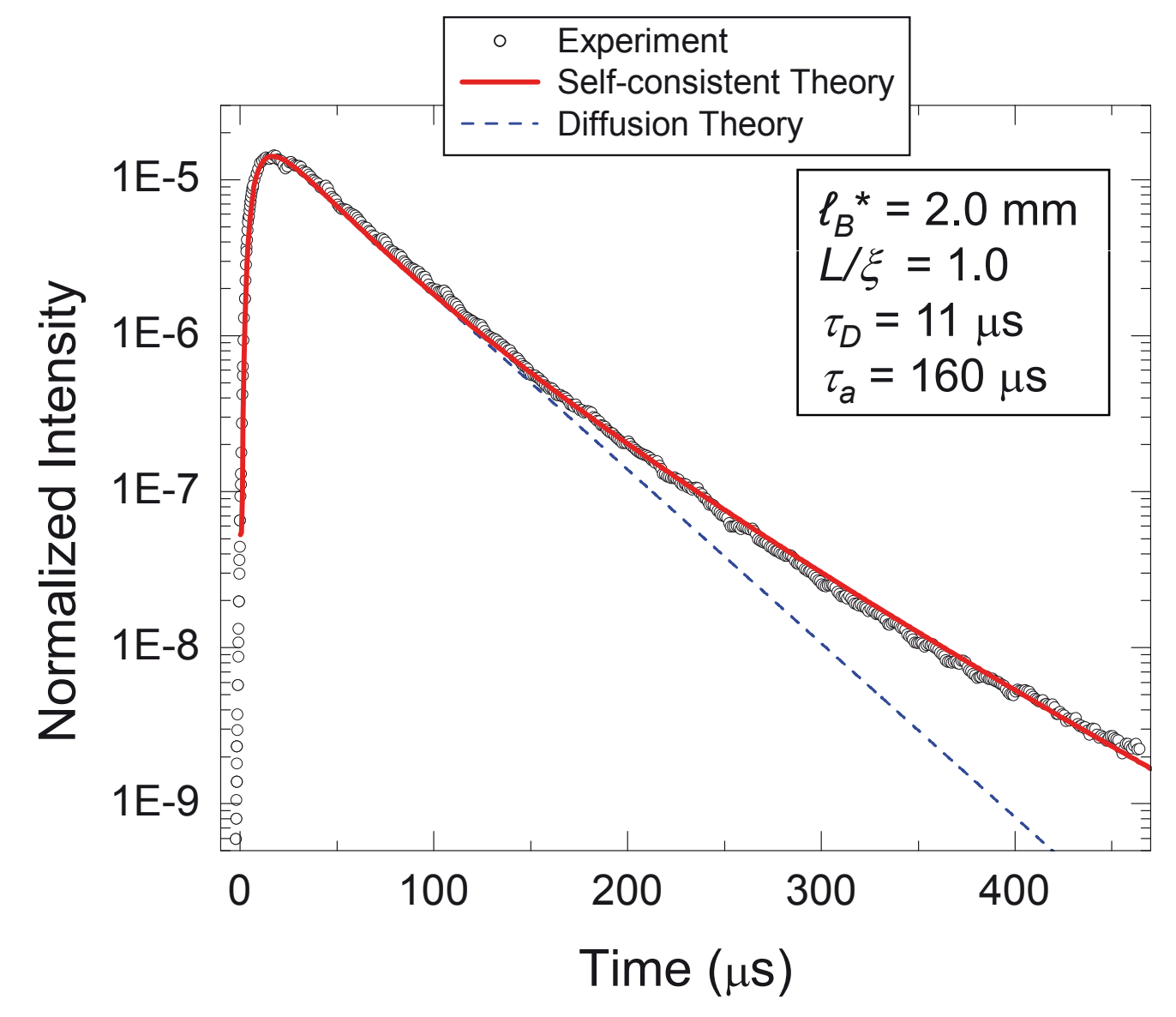

Excellent fit of $I(t)$, at all propagation times, with the predictions of the self-consistent theory [Skipetrov \& van Tiggelen (2006)] provides strong (but indirect) evidence for the localization of sound in these 3D samples

Question: Can we find more direct evidence for 3D localization in ultrasonic experiments? 


\section{Transverse confinement ("transverse localization in 3D")}

Experiment (displaced point source technique):

- Point source (focusing transducer + small aperture)

- Point detector, placed a transverse distance $\rho$ away

- Scan $x-y$ position of the sample to determine $I(\rho, t)$.

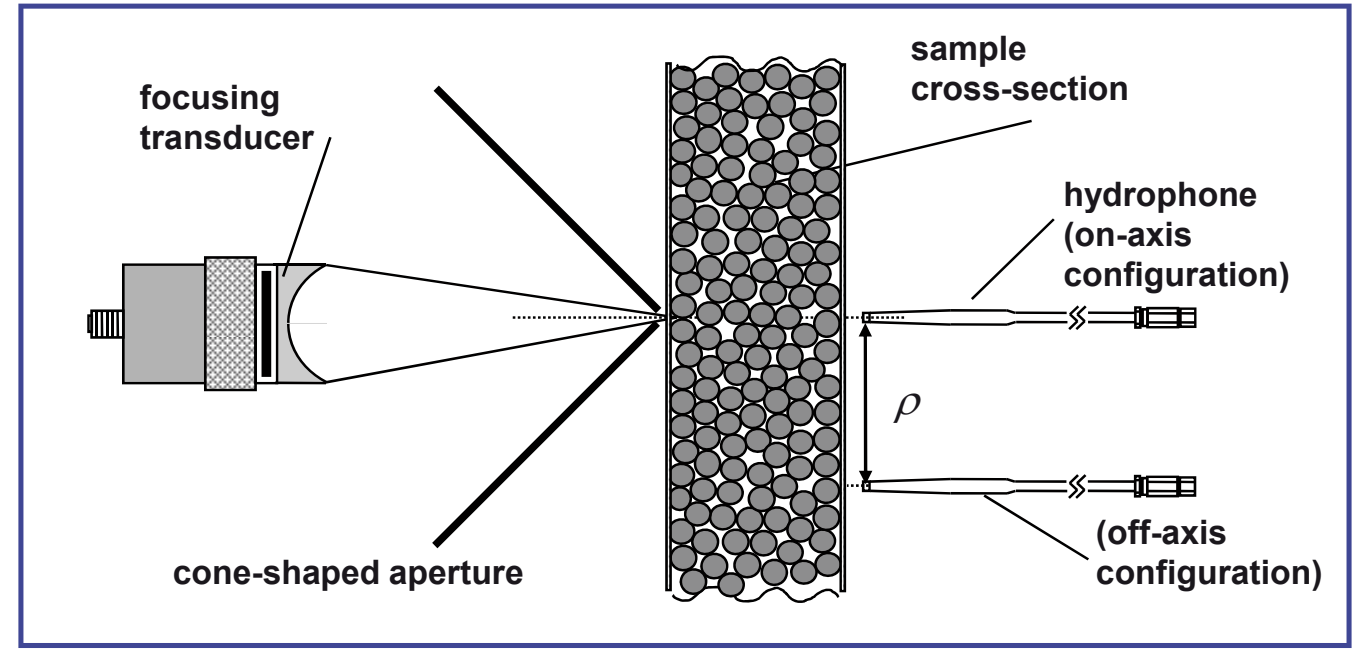

The ratio $I(\rho, t) / I(0, t)$ probes the transverse growth (dynamic spreading) of the intensity profile.

- Diffuse regime - measure the effective width of the "diffuse halo", which provides a method of measuring $D$ independent of boundary conditions and absorption. [Page et al., Phys. Rev. E 52, 3106 (1995)]

$$
\begin{array}{r}
\frac{I(\rho, t)}{I(0, t)}=\exp \left[-\rho^{2} / 4 D t\right] \equiv \exp \left[-\rho^{2} / w^{2}(t)\right] \quad \text { so the effective width } w(t) \text { is } \\
w^{2}(t)=-\frac{\rho^{2}}{\ln [I(\rho, t) / I(0, t)]}=4 D t
\end{array}
$$


Diffuse regime -the effective width of the "diffuse halo" grows linearly in time Data (from 1995) on a suspension of glass beads in water $(k \ell \sim 7)$ [Page et al., Phys. Rev. E 52, 3106 (1995)]
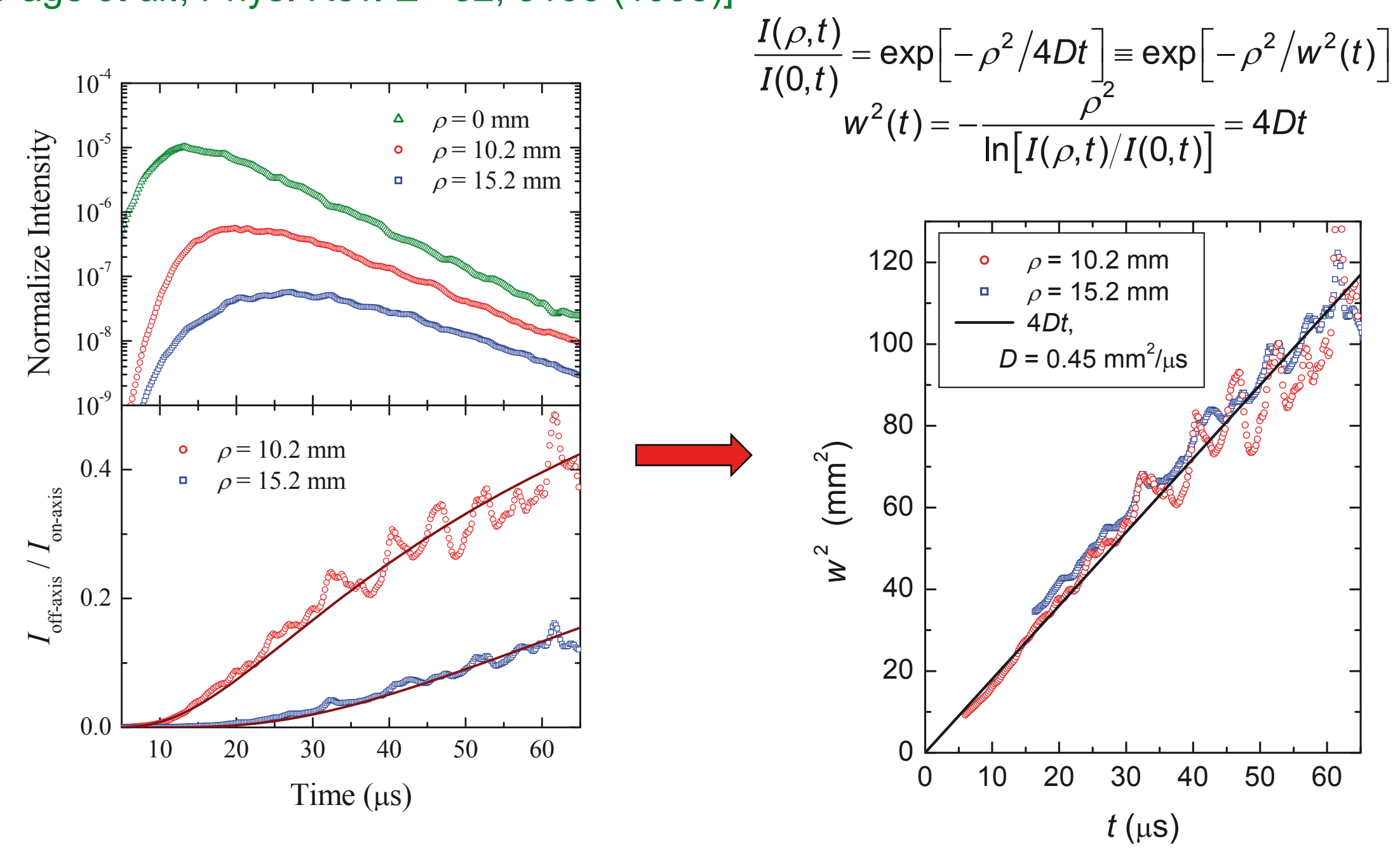

Measure $D_{B}$ independent of boundary conditions and absorption. 
Question: What happens to $I(\rho, t) \& w(t)$ in the localization regime?
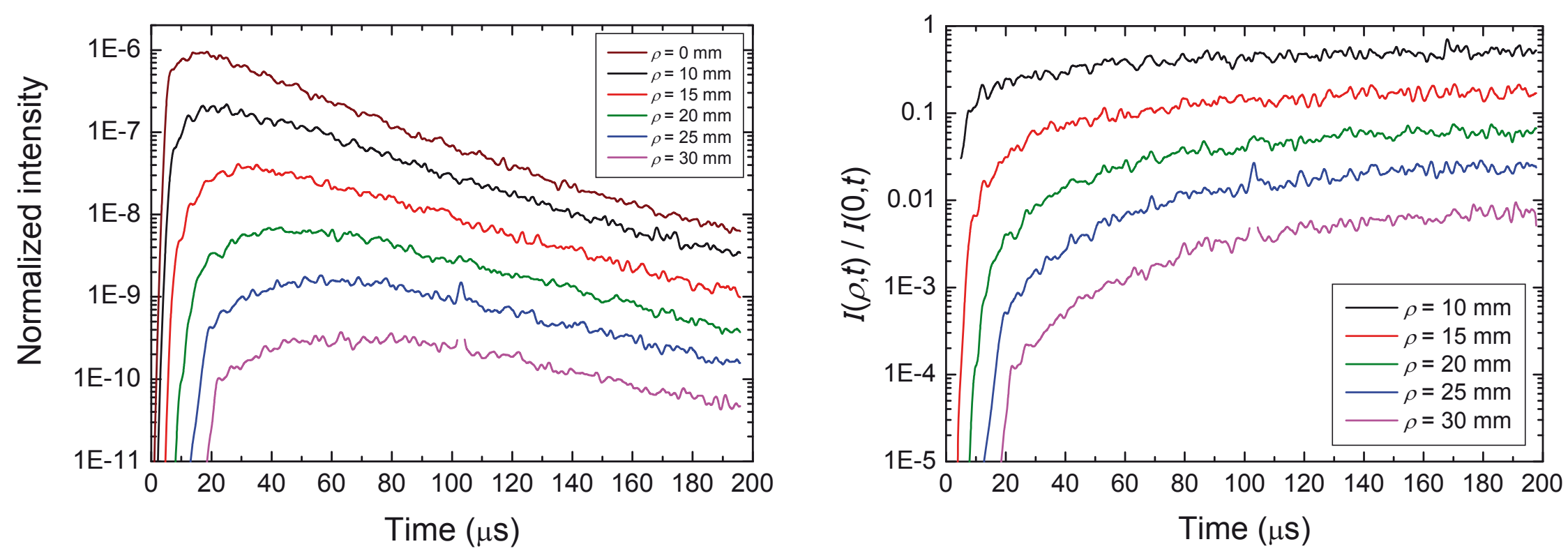

Dynamic transverse width at $2.4 \mathrm{MHz}$ :

Localization dramatically inhibits the expansion of the intensity profile in the transverse direction.

$$
\frac{I(\rho, t)}{I(0, t)}=\exp \left[-\rho^{2} / w^{2}(t)\right]
$$

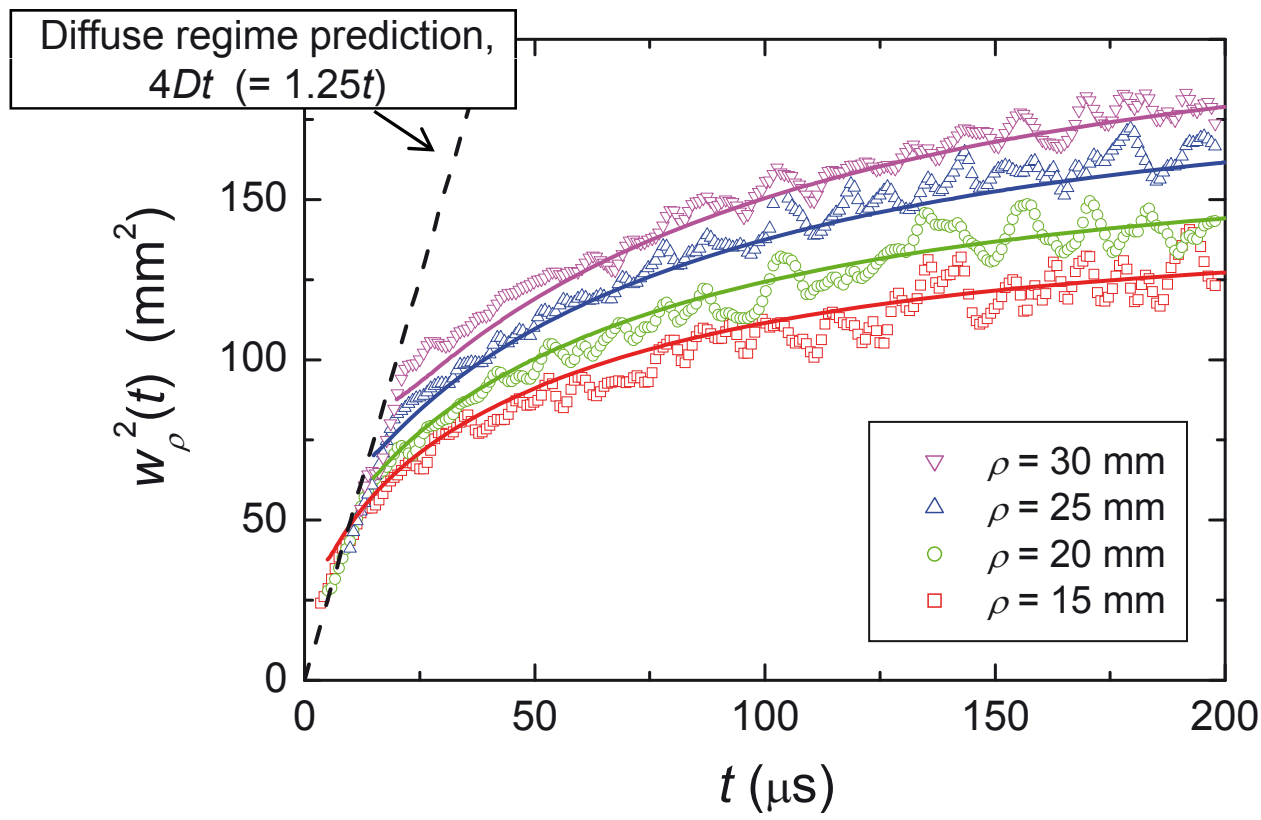


Quantitative analysis of the dynamic transverse width, $w(t)$ :

- Fit the data using the new self consistent theory that allows for the position dependence of the renormalized diffusion coefficient in 3D.

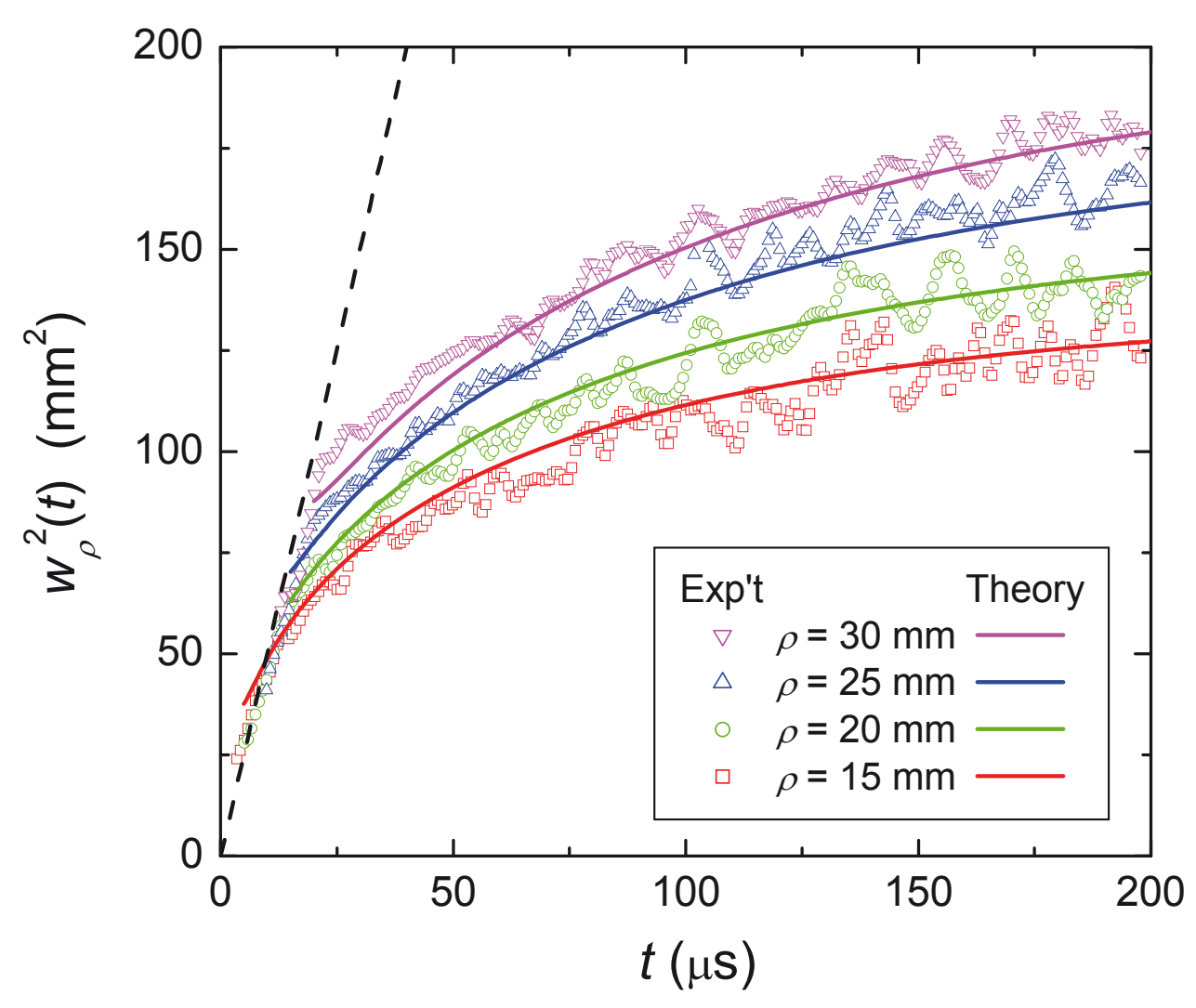

- Excellent fit for all four $\rho$ with:

$\ell_{B}{ }^{*}=2.0 \mathrm{~mm}$

$L / \xi=1.0$

$\tau_{D}=17 \mu \mathrm{s}$

( $\tau_{a}$ cancels in ratio)

- Fit is more sensitive to $\xi$ than plane wave $I(t)$

- Again, find $\xi>0 \Rightarrow$ classical wave localization is convincingly demonstrated in this 3D "phononic" mesoglass.

- First direct measurement and theory for the transverse structure of localized waves in 3D. Find $w \sim 12-14 \mathrm{~mm} \sim \xi$ for this sample 
3D Transverse Localization: this animation (prepared by Sergey Skipetrov) shows the "freezing" of the transverse profile at long times (saturation of $I(\rho, t) / I(\rho, 0)$ occurs for $t>t_{\mathrm{loc}} \sim 100 \mu \mathrm{s}$ in this case.)

Transverse Iocalization in 3D

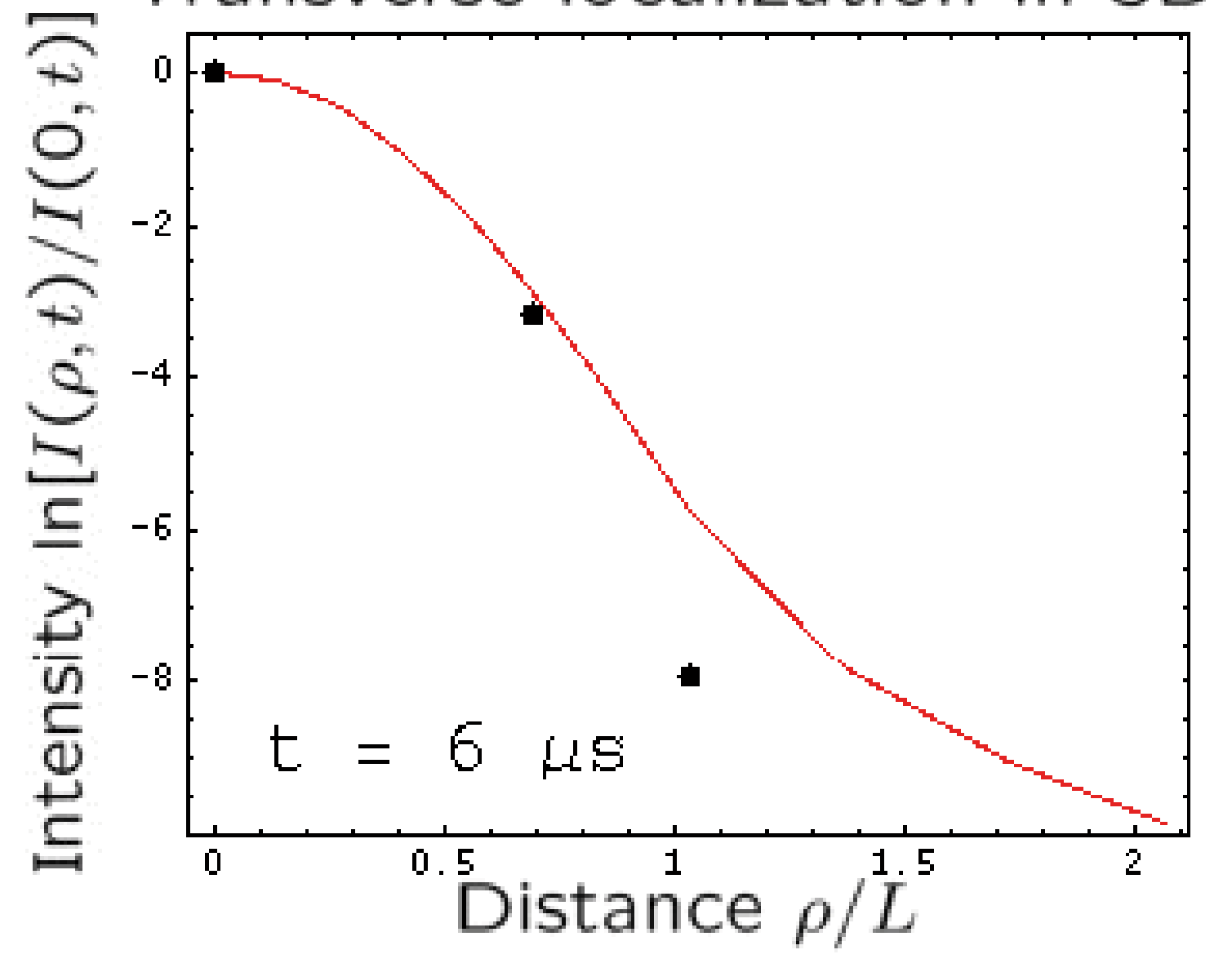

This animation may be viewed at http://www.physics.umanito ba.ca/ jhpage/HTML/locali zation/localization2.html 
Decrease of $I(\rho, t)$ with transverse distance $\rho$ is not Gaussian $\Rightarrow$ Near the mobility edge $\left(k \ell /(k \ell)_{\mathrm{c}}=0.99\right.$ for this sample at this frequency), $w$ varies somewhat with transverse displacement $\rho$.

The self-consistent theory (solid curves) captures the experimentally observed dependence of $w(t)$ on $\rho$ very well.

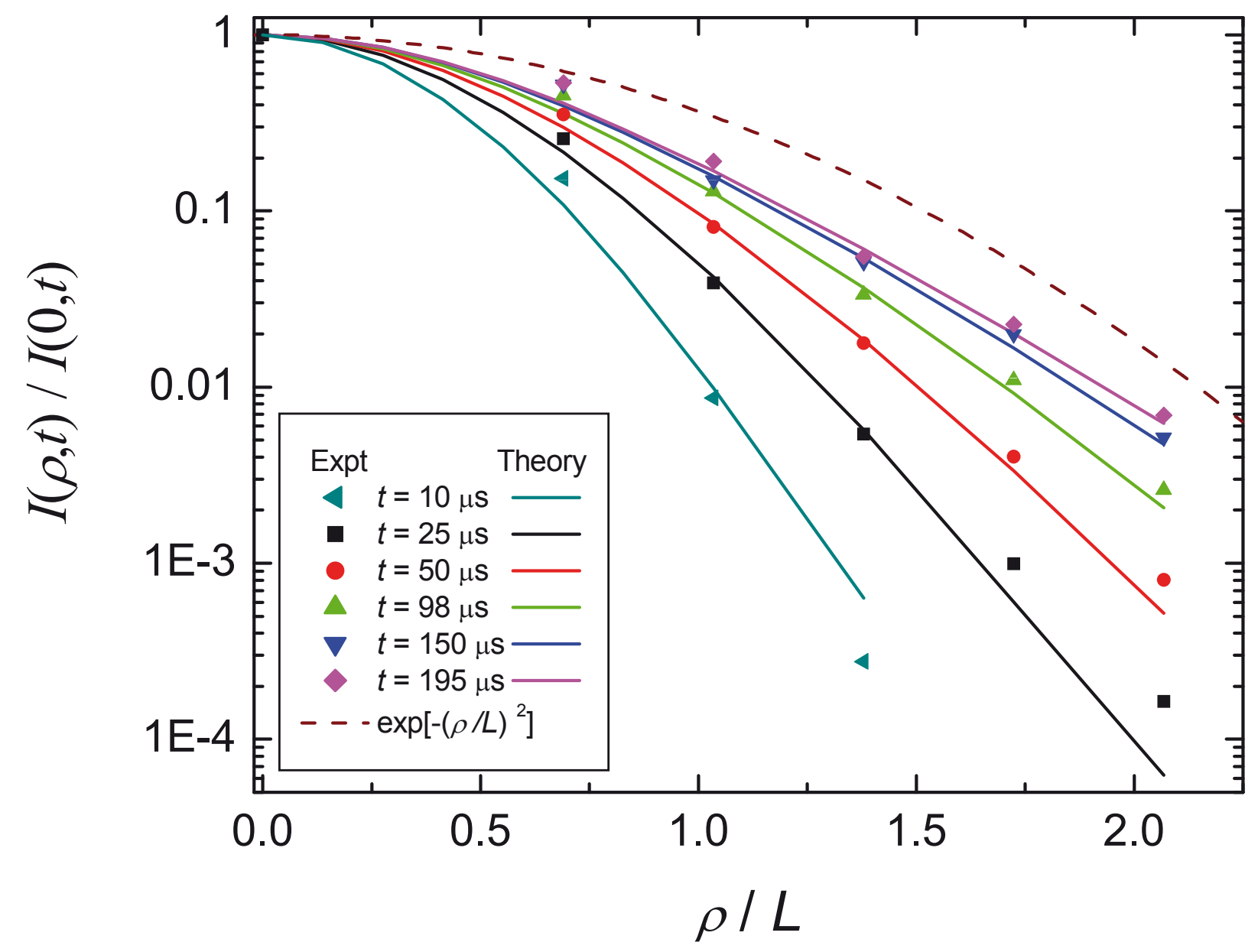


Question: What determines the magnitude of the dynamic transverse width $w_{\rho}(t)$ ?

- For thick samples, $w$ becomes independent of $\rho$.

- Behaviour at long times: SC theory predictions for the saturated width when $L \gg$ > :

$$
w^{2}(t \rightarrow \infty) \approx 2 L \xi(1-\xi / L)
$$

[Cherroret, Skipetrov and van Tiggelen, aiXiv:0810.0767v1]
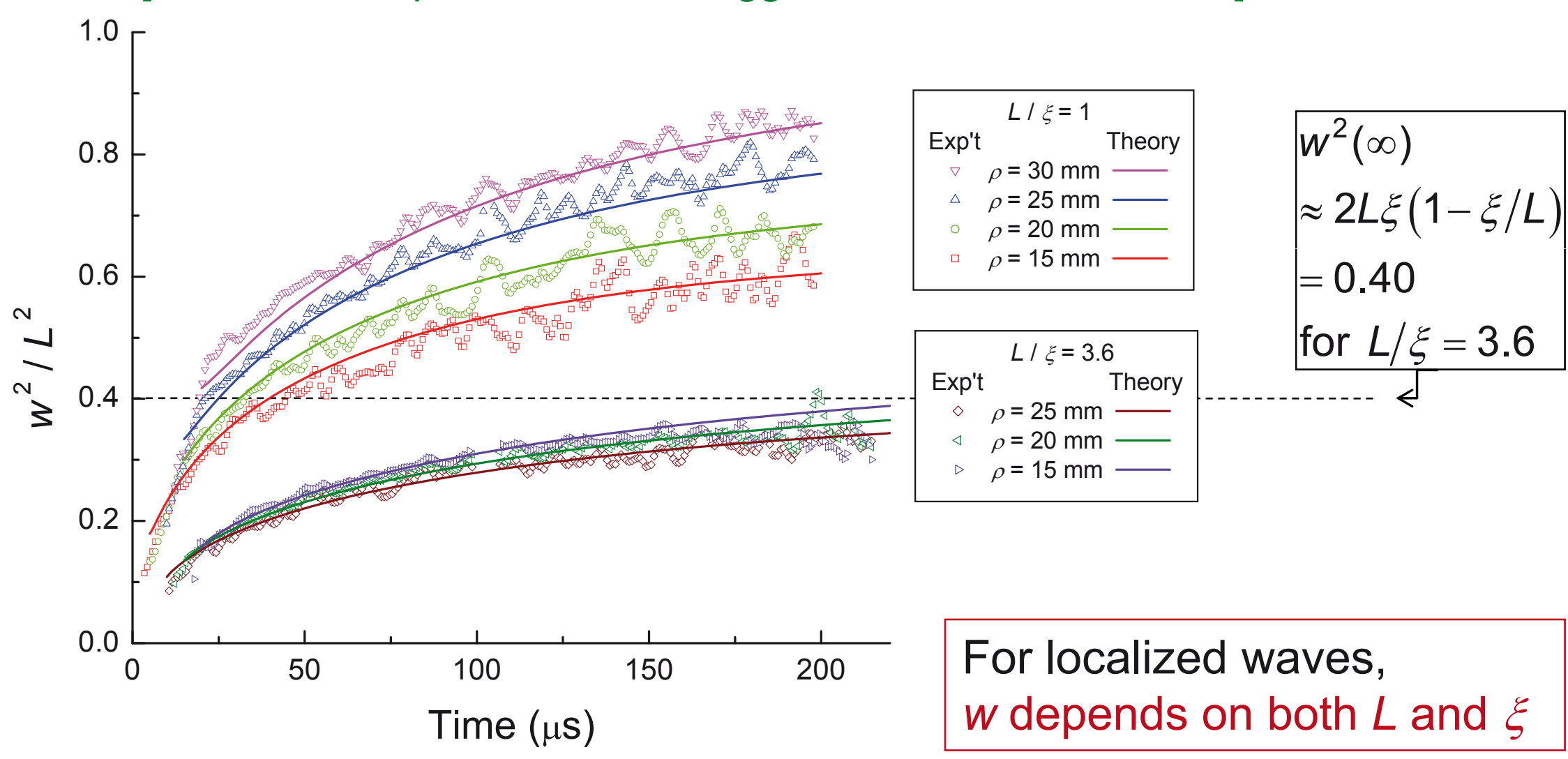
The saturation of $w(t)$ at long times is predicted even at the mobility edge [Cherroret, Skipetrov and van Tiggelen, arXiv:0810.0767v1].

Numerical calculations using the dynamic self-consistent theory:

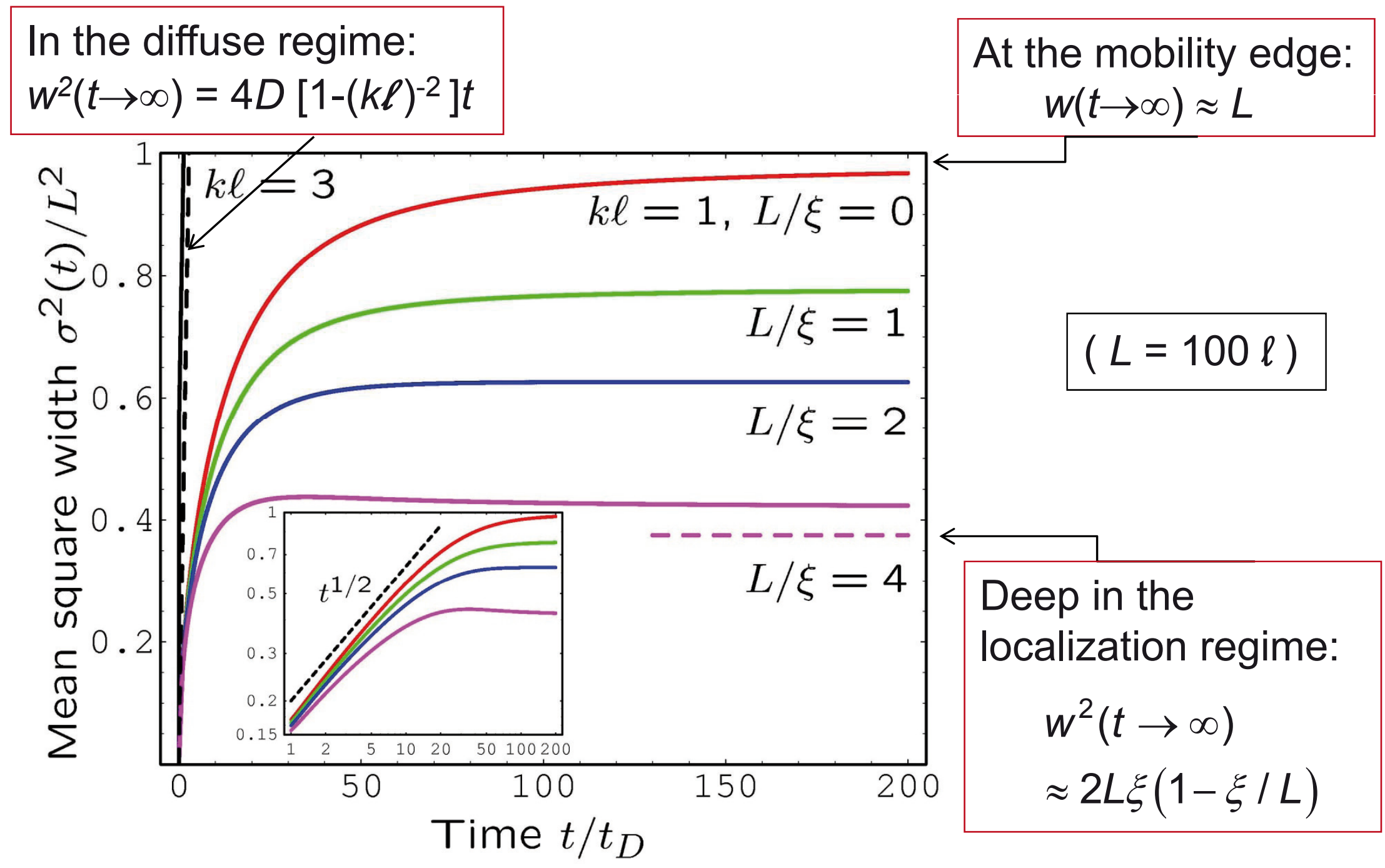


Similar trends are seen in the experiments (for $t / \tau_{D}<20$ )

- Compare three representative samples with different amounts of disorder (same measuring frequency $f=2.4 \mathrm{MHz}$ ).

$L=14.5 \mathrm{~mm}, \xi=15 \mathrm{~mm} ; L=23.05 \mathrm{~mm}, \xi=12 \mathrm{~mm} ; L=23.5 \mathrm{~mm}, \xi=6.5 \mathrm{~mm}$;

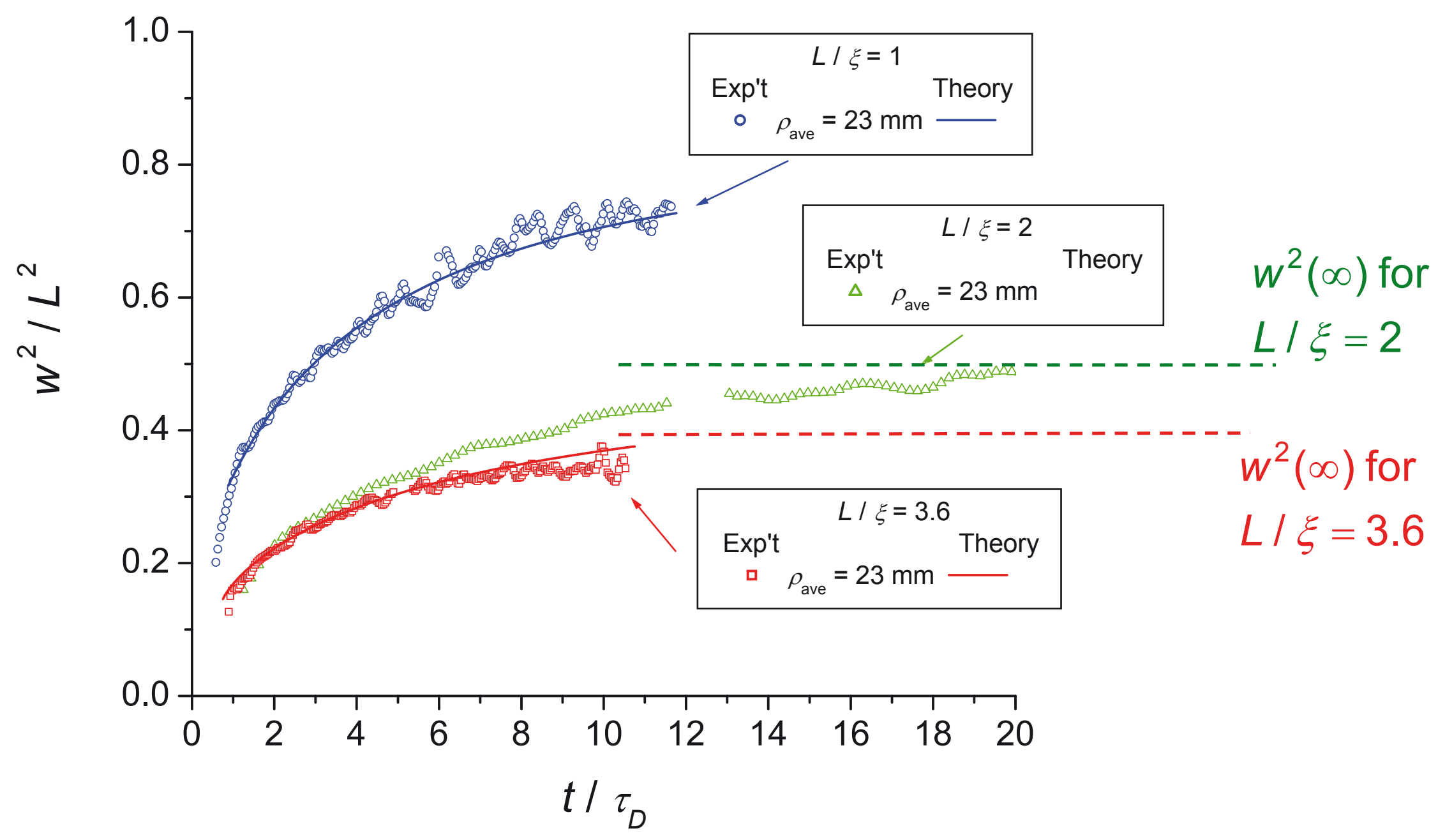


What happens when we vary the frequency?

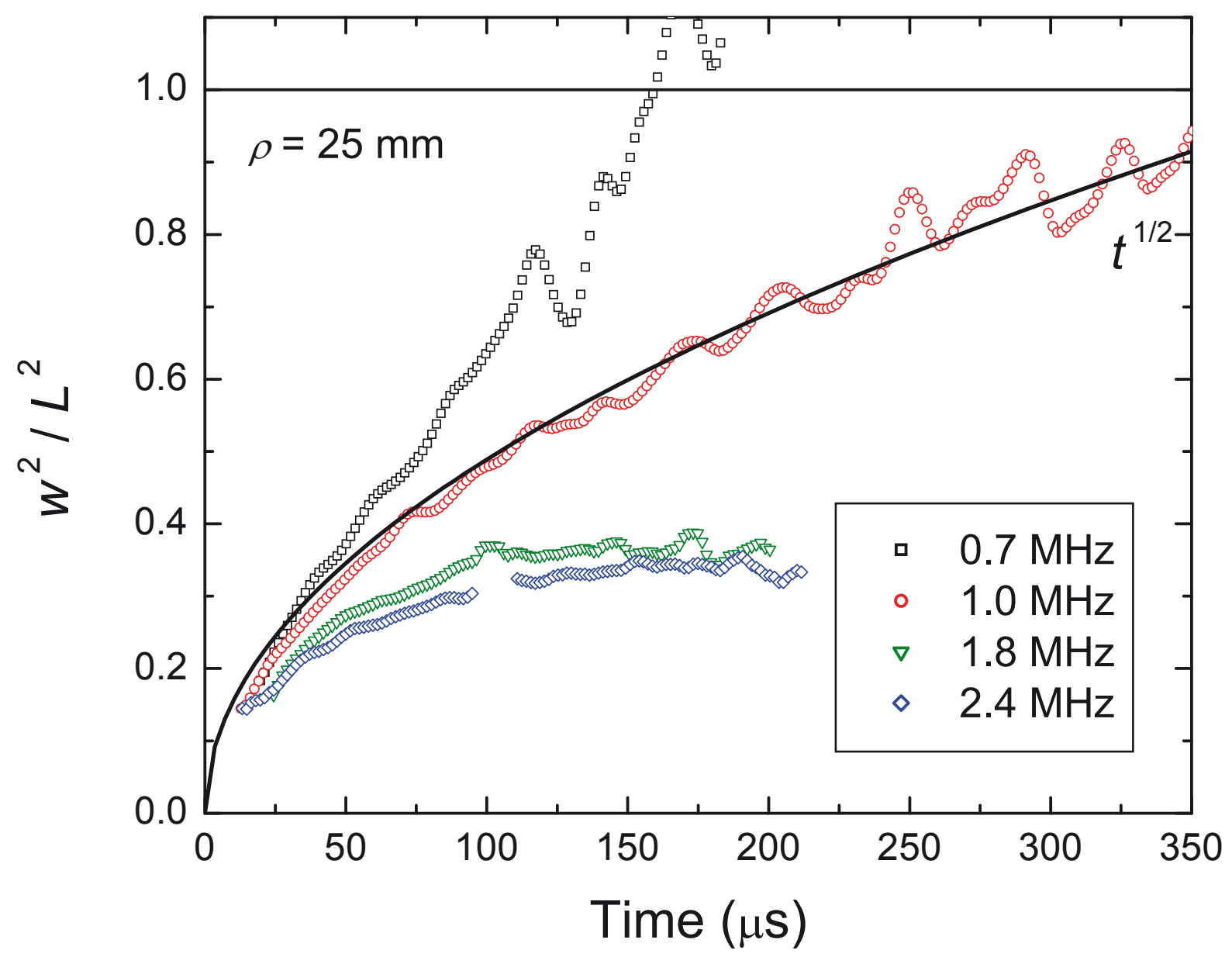

At 0.7 and $1.0 \mathrm{MHz}, w^{2}(t)$ does not saturate $\Rightarrow$ above the mobility edge.

(at $0.7 \mathrm{MHz}$, the time dependence is almost linear)

Should be feasible to measure $\xi$ as the mobility edge is approached 


\section{What happens when we vary the frequency?}

Plot on log scales to show the time dependence

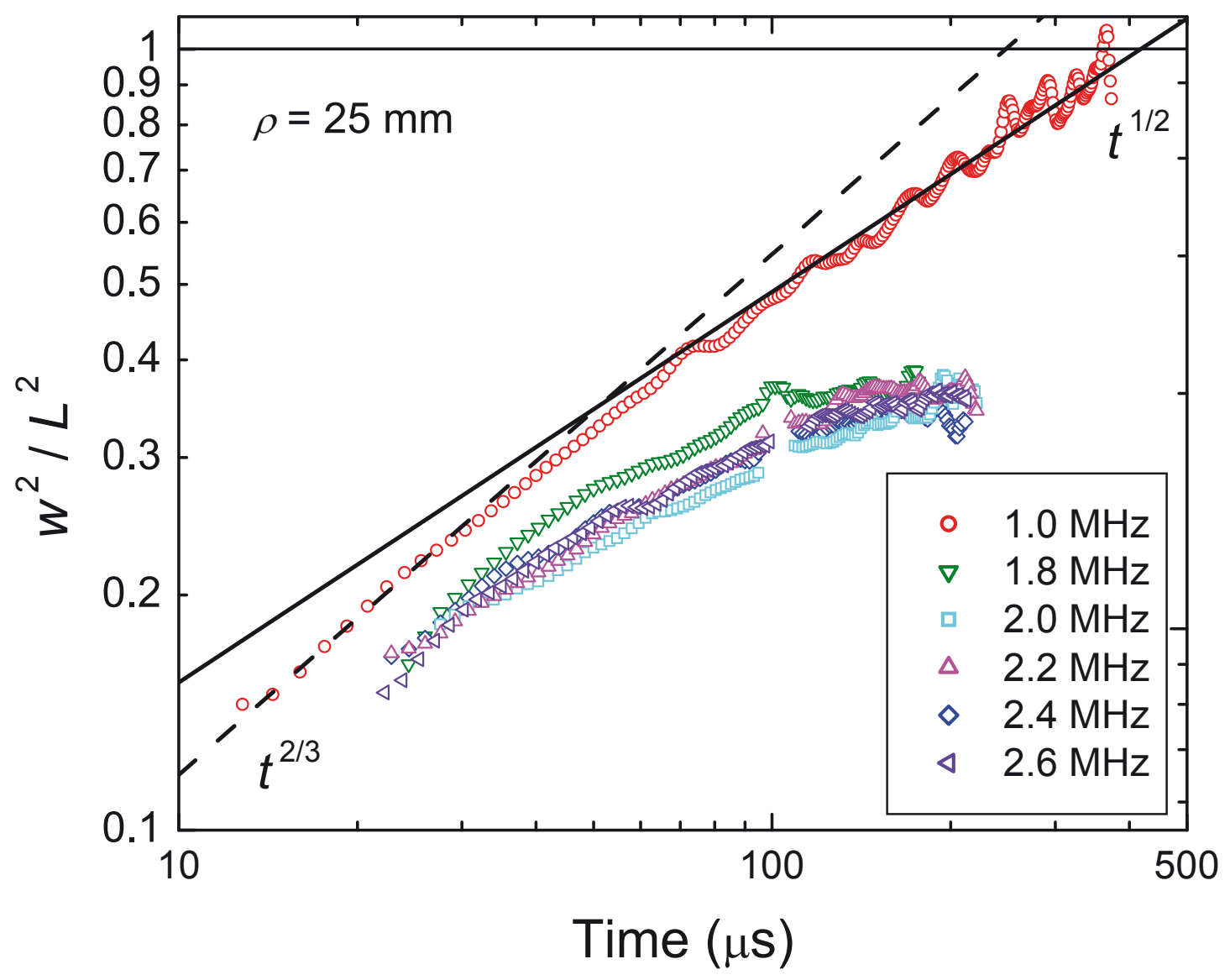

Near the mobility edge, we see

$w^{2}(t) \propto t^{2 / 3}$ for $t<\tau_{D} \quad \&$

$w^{2}(t) \propto t^{1 / 2}$ for a limited range of $t>\tau_{D}$

Agrees with estimates of $w^{2}(t)$ using the selfconsistent theory. 


\section{Summary: Transverse confinement (3D transverse localization)}

- The dynamic transverse width $w^{2}(t)$ has completely different properties for diffuse and localized modes

Diffuse: $w^{2}(t) \propto t$ and increases without bound.

Localized: $w^{2}(t)$ saturates at long times.

At the mobility edge: $\quad w(t \rightarrow \infty) \approx L$

Deep in the localization regime: $w^{2}(t \rightarrow \infty) \approx 2 L \xi(1-\xi / L)$

- $w^{2}(t)$ is independent of absorption $\rightarrow$ its measurement (for any kind of wave) provides a valuable method for assessing whether or not the waves are localized. (No risk of confusing absorption with localization.)

- $w^{2}(t)$ can be used to measure the localization length $\xi$.

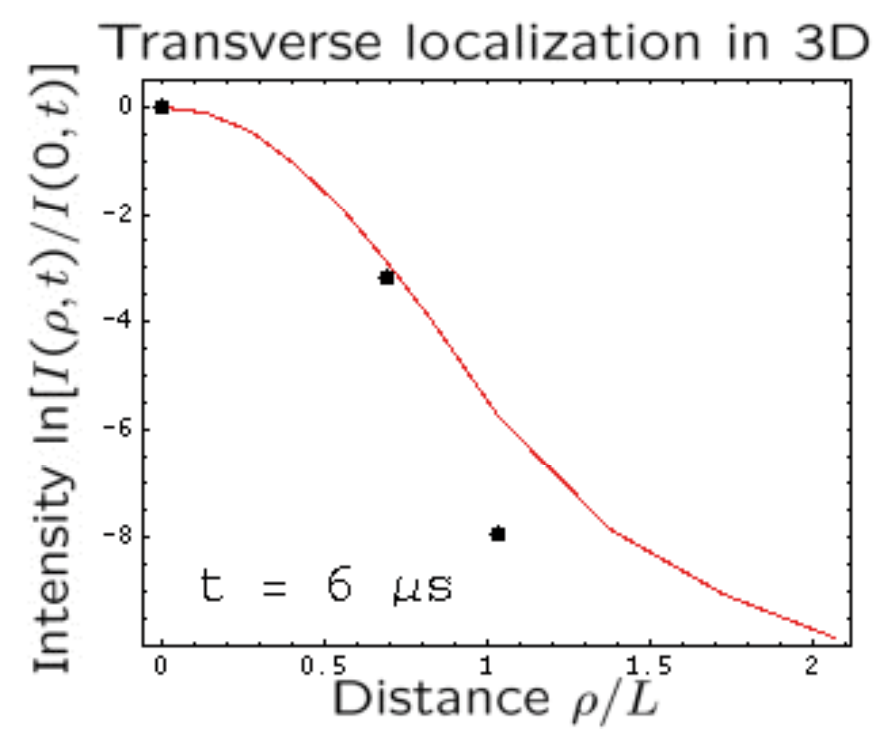


IV. Statistical approach to the localization of sound:
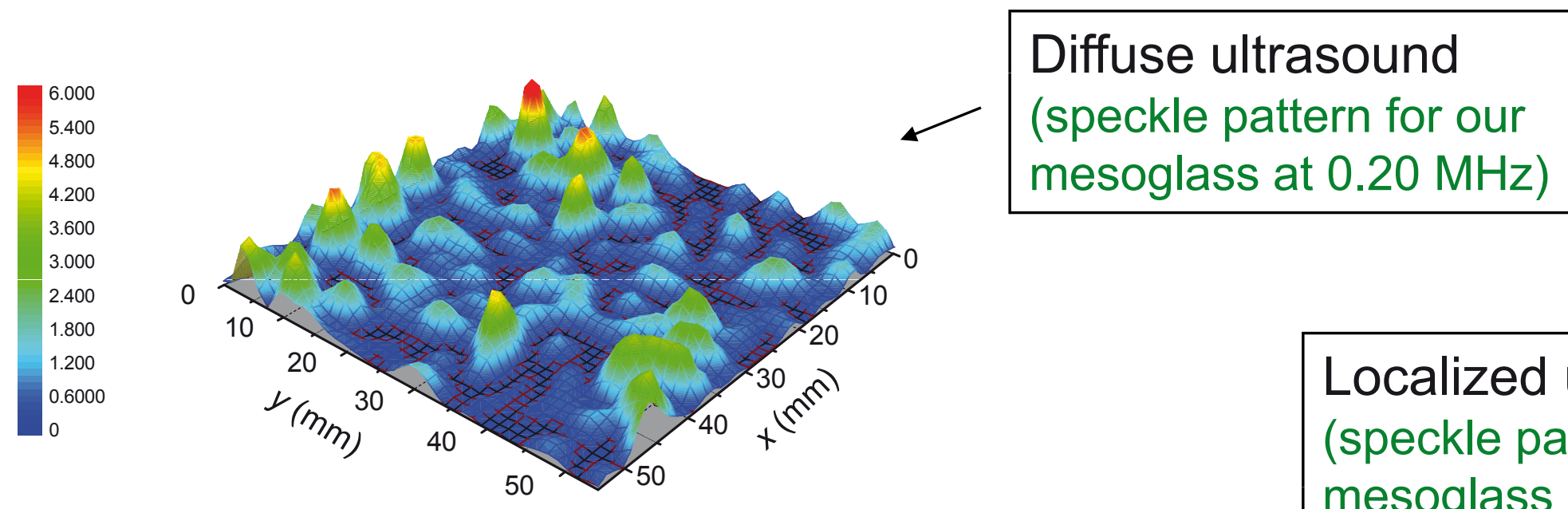

Large fluctuations in the transmitted intensity are characteristic of localized waves.

Signatures of these fluctuations are seen in:

- Near field speckle pattern

- Intensity distribution $P(I /\langle I\rangle)$

- Variance

- Multifractality

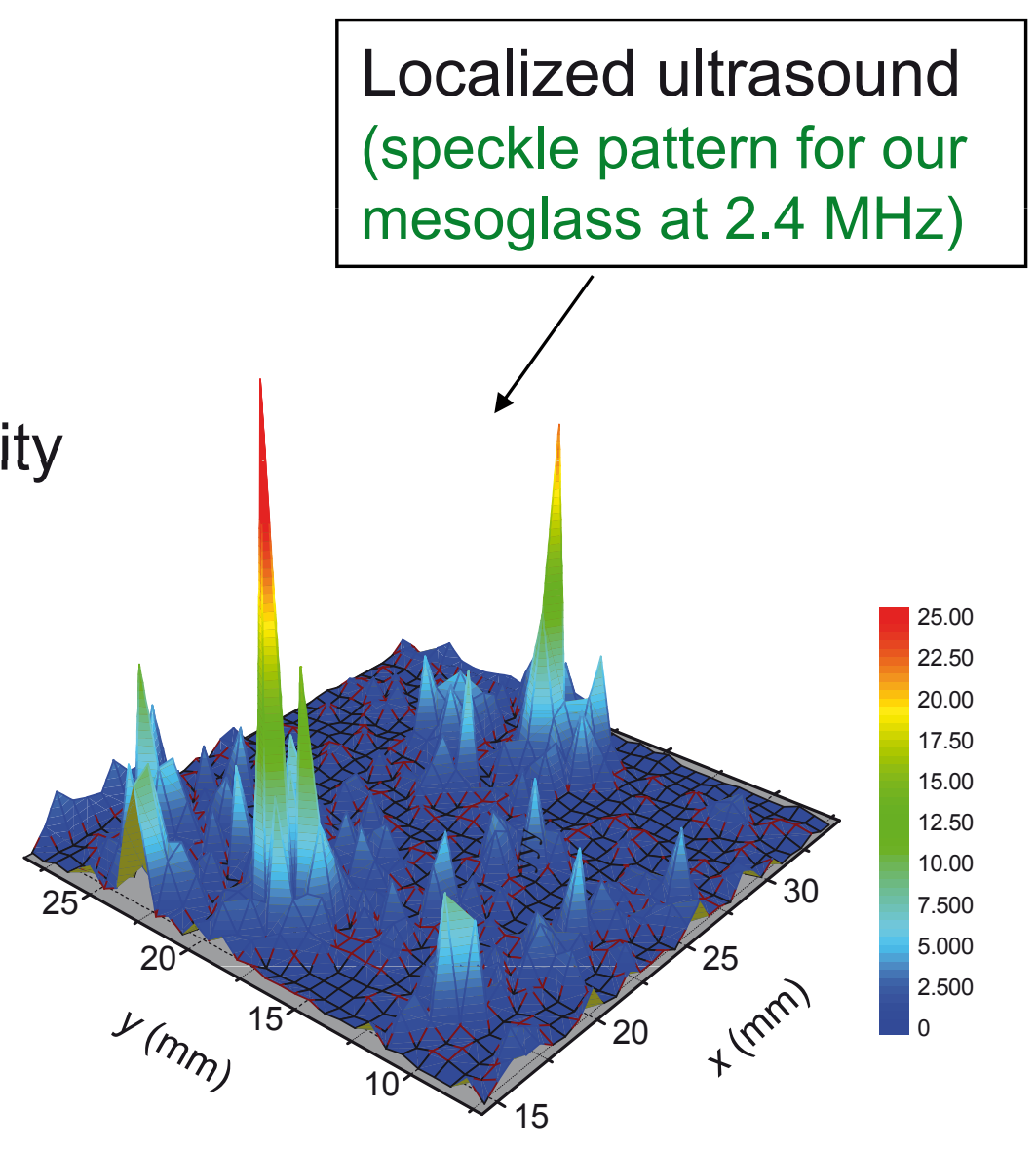


Transmitted intensity distributions for our mesoglass:

Measure the intensity $I$ at each point in the near field speckle pattern when the sample is illuminated on the opposite side with a broad beam. When $I$ is normalized by its average value to get $\hat{I}=I /\langle I\rangle$, its distribution is universal.

(a) Data at $0.20 \mathrm{MHz}$

Rayleigh distribution:

(random wave fields described

by circular Gaussian statistics)

$$
P(\hat{I})=\exp (-\hat{I})
$$

Leading order correction to Rayleigh statistics due to interference (no absorption) [Nieuwenhuizen \& van Rossum, PRL 74, 2674 (1995)] ( $g^{\prime}=$ dimensionless conductance):

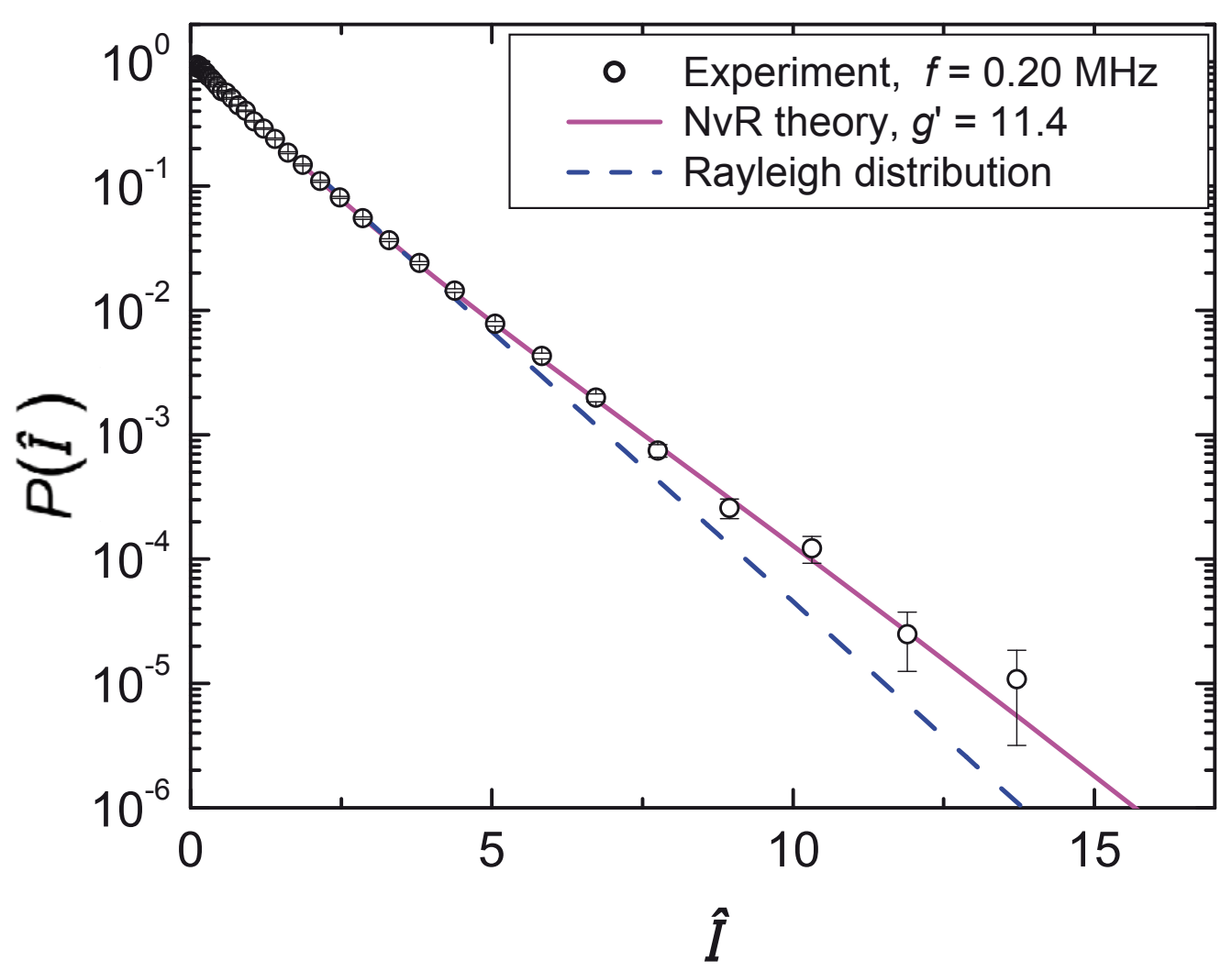

$$
P(\hat{I})=\exp (-\hat{I})\left[1+\frac{1}{3 g^{\prime}}\left(\hat{I}^{2}-4 \hat{I}+2\right)\right]
$$

Find $g^{\prime}=11.4>1$

$\Rightarrow$ modes are extended 
Transmitted intensity distributions for our mesoglass:

(b) Near $2.4 \mathrm{MHz}$ (upper part of intermediate frequency regime), find very large departures from Rayleigh Statistics

Fit the entire distribution to predictions by van Rossum and Nieuewenhuizen [Rev. Mod. Phys. 71, 313] for a slab geometry in $3 \mathrm{D}$ (red curve). Remarkable agreement with experiment.

The tail of intensity distribution obeys a stretched exponential distribution

$$
P(\hat{I}) \sim \exp \left(-2 \sqrt{g^{\prime} \hat{I}}\right)
$$

( $g^{\prime}$ is the effective dimensionless conductance.) Find $g^{\prime}=0.80<1$, indicating localization.

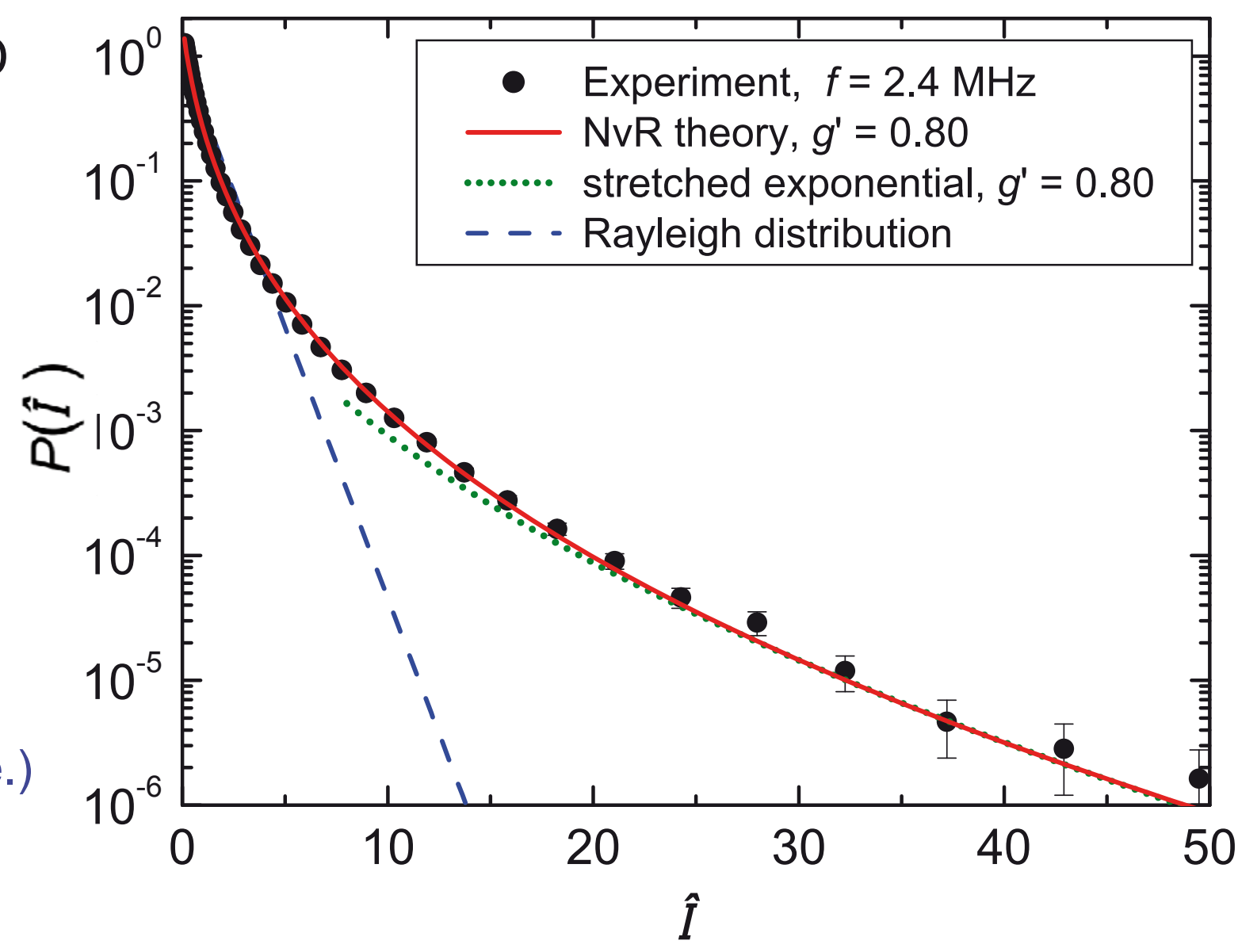


Variance of the transmitted intensity - a simpler way to measure the dimensionless conductance $g^{\prime}$ :

Chabanov et al. [Nature 404, 850 (2000)] have proposed that localization is achieved when the variance of the normalized total transmitted intensity , $\hat{T}=T /\langle T\rangle$ satisfies

$$
\operatorname{var}(\hat{T}) \equiv \frac{\left\langle\delta T^{2}\right\rangle}{\langle T\rangle^{2}}=\frac{2}{3 g^{\prime}} \geq \frac{2}{3}
$$

whether absorption is present or not. This corresponds to the localization condition $g^{\prime} \leq 1$.

But $\operatorname{var}(\hat{T})$ and $\operatorname{var}(\hat{I})$ are related: $\quad \operatorname{var}(\hat{I})=2 \operatorname{var}(\hat{T})+1$

Then, the Chabanov-Genack localization criterion gives $\operatorname{var}(\hat{I}) \geq 7 / 3$

e.g., for our data at $2.4 \mathrm{MHz}$ :

Measure $\operatorname{var}(\hat{I})=2.74 \pm 0.09 \Rightarrow g^{\prime}=\frac{4}{3[\operatorname{var}(\hat{I})-1]}=0.77 \pm 0.4$

Excellent agreement with $g^{\prime}=0.80 \pm 0.08$ measured from $P(\hat{I})$

Additional evidence that the modes are localized above $\sim 2 \mathrm{MHz}$. 
Time dependence of the speckle intensity variance:
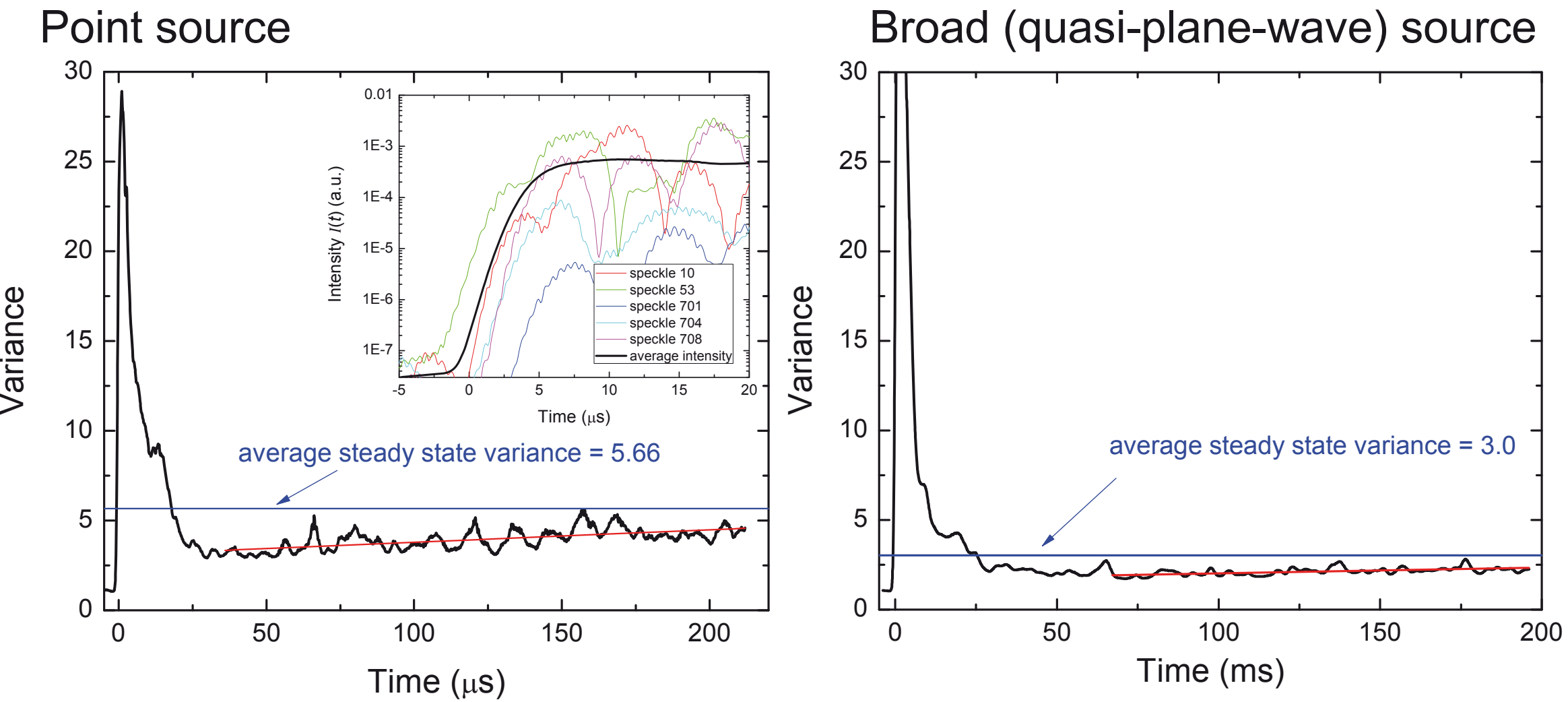

- Large peak in variance at early times - due to arrival time fluctuations.

- Variance increases slowly with time at longer times (slower growth than in quasi 1D - poster by Wang et al.).

- Both variance and its growth with time are larger for a point source.

- Time-dependent variance is less than the stationary variance for the range of times measured. 
Time dependence of the speckle intensity variance:

Data are consistent with theoretical estimates by Sergey Skipetrov, based on a mode picture of wave propagation:

$$
\psi(\mathbf{r}, t)=\sum A_{n} \psi_{n}(\mathbf{r}) e^{-i \omega_{n} t-\frac{1}{2} \Gamma_{n} t}
$$

(quasi-mode frequencies $\omega_{n}$, lifetimes $\Gamma_{\mathrm{n}}-$ for $P(\Gamma)$, see

Skipetrov \& van Tiggelen, PRL (2006))

Assuming uncorrelated modes, an estimate of the variance gives

$$
\delta^{2}(t)=\operatorname{var}(\tilde{I}(t)) \approx 1+\frac{1}{N}\left[\left(1+\delta_{\text {stationary }}^{2}\right) f\left(t / t_{\xi}, L / \xi\right)-2\right]
$$

where $t_{\xi}=\xi^{2} / D_{B} \quad$ and

$$
\begin{aligned}
f\left(t / t_{\xi}, L / \xi\right) & =\frac{\left\langle e^{-2 \Gamma t}\right\rangle}{\left\langle e^{-\Gamma t}\right\rangle^{2}} \\
& = \begin{cases}a+b \sqrt{t / t_{\xi}}, & t \rightarrow 0 \\
c+d\left(t / t_{\xi}\right), & t \rightarrow \infty\end{cases}
\end{aligned}
$$

Predictions (valid at long times, $t>>\tau_{D}$ ) for typical experimental parameters reproduce the main features in the data

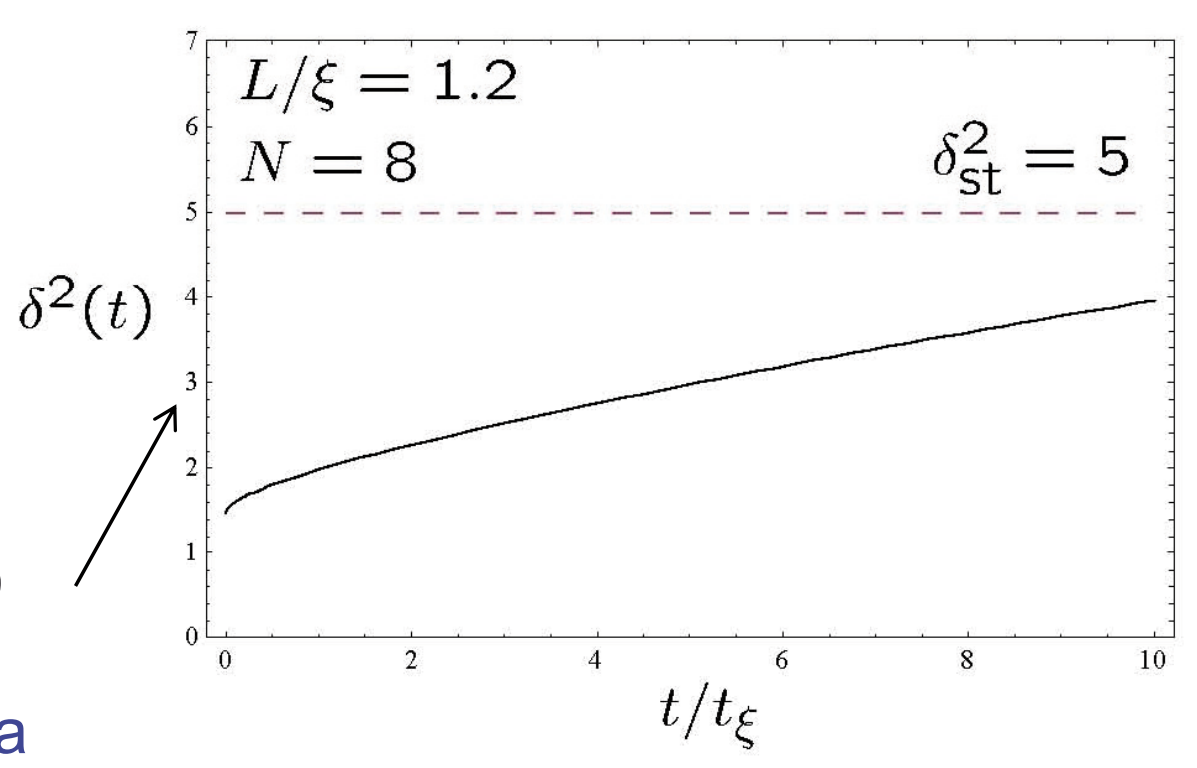


Multifractality (MF) of the wavefunction (with Sanli Faez, Ad Lagendijk): [Faez et al., PRL 103, 155703 (2009) ]

Key idea: Large fluctuations $\Rightarrow$ the moments of the wave function intensity

$$
I(\mathbf{r})=\left|\psi^{2}(\mathbf{r})\right| / \int\left|\psi^{2}(\mathbf{r})\right| d^{d} r
$$

may depend anomalously on length scale near the Anderson transition, exhibiting multifractal behaviour .

(MF $\Rightarrow$ each moment scales with a different power- law exponent).

- Many theoretical predictions, but almost no experimental evidence

Key result: By examining the scaling of the generalized Inverse Participation Ratios, the probability density function and the "singularity spectrum", we have shown that the spatial structure of the ultrasound wavefunctions exhibits multifractality near the Anderson transition.

Don't miss the talk by Sanli Faez tomorrow!
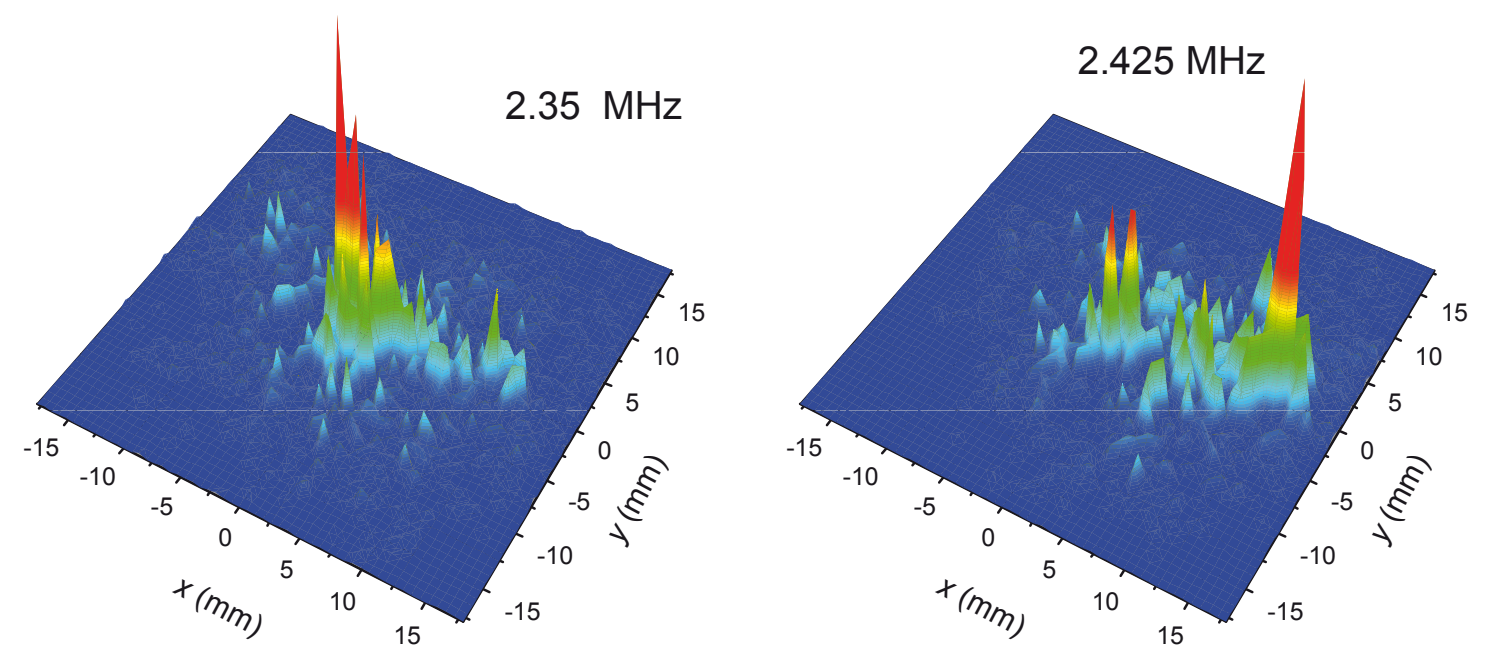


\section{Statistics - Summary}

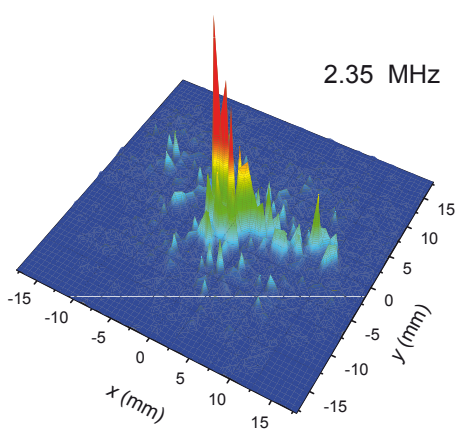

- Large fluctuations in the transmitted intensity for localized modes:

$$
\begin{aligned}
& \text { non-Rayleigh statistics } \\
& \text { large variance, } \operatorname{var}(\hat{I})
\end{aligned} \quad \rightarrow g<1
$$

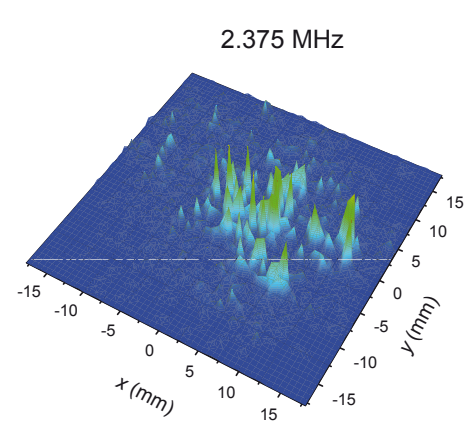

- First experimental observations of wavefunction multifractality near the Anderson transition:

$$
\begin{aligned}
& \text { scaling of the gIPR, }\left\langle P_{q}\right\rangle \sim(L / b)^{-\tau(q)} \\
& \text { probability density function }
\end{aligned}
$$

(PDF is log normal)

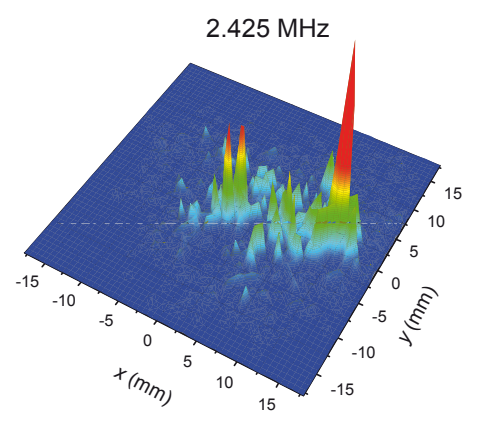

singularity spectrum, $f(\alpha) \quad\left(\alpha_{\text {peak }}>d\right)$

Listen to Sanli Faez's talk for more on multifractality... 


\section{Conclusions}

We have used ultrasonic experiments and predictions of the selfconsistent theory of dynamics of localization to demonstrate the localization of elastic waves in a 3D disordered mesoglass.

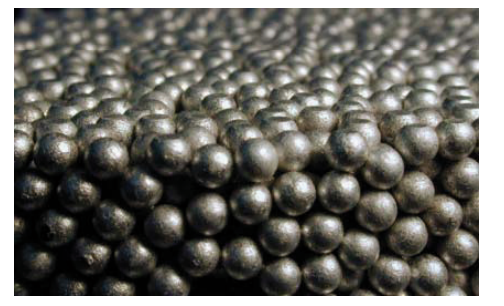

Localization signatures

$>$ Time dependent transmitted intensity $I(t)$

$\rightarrow$ non-exponential decay of $I(t)$ at long times.

$>$ Transverse confinement $\rightarrow$ first direct

measurements and theory for $I(\rho, t)$, showing how localization cuts off the transverse spreading of the multiple scattering halo.

$W^{2}(t)$ is independent of absorption and depends on the localization length $\xi$ (and $L$ )

$>$ non-Rayleigh statistics and large variance of the ${ }^{2} / m_{m}$ transmitted intensity $\hat{I}$; wavefunction multifractality.

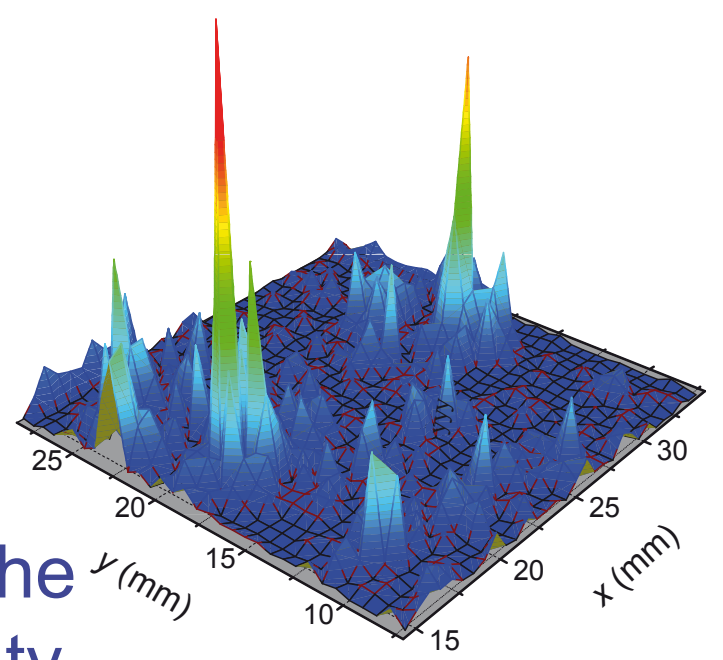

dimensionless conductance $g^{\prime}=0.8<1(2.4 \mathrm{MHz})$

Transverse confinement is a powerful new approach for guiding investigations of 3D Anderson localization for any type of wave. 